\title{
Learning a Test Oracle towards Automating Image Segmentation Evaluation
}

\author{
By
}

\section{Kambiz Frounchi}

A thesis submitted to the Faculty of Graduate Studies and Research in partial fulfillment of the requirements for the degree of Master of Applied Science

Ottawa-Carleton Institute of Electrical and Computer Engineering Department of Systems and Computer Engineering Carleton University Ottawa, Ontario, Canada 


$\begin{array}{ll}\begin{array}{l}\text { Library and } \\ \text { Archives Canada }\end{array} & \begin{array}{l}\text { Bibliothèque et } \\ \text { Archives Canada }\end{array} \\ \begin{array}{l}\text { Published Heritage } \\ \text { Branch }\end{array} & \begin{array}{l}\text { Direction du } \\ \text { Patrimoine de l'édition }\end{array} \\ \begin{array}{l}\text { 395 Wellington Street } \\ \text { Ottawa ON K1A 0N4 } \\ \text { Canada }\end{array} & \begin{array}{l}\text { 395, rue Wellington } \\ \text { Ottawa ON K1A 0N4 } \\ \text { Canada }\end{array}\end{array}$

Your file Votre référence ISBN: 978-0-494-47512-6 Our file Notre référence ISBN: 978-0-494-47512-6

NOTICE:

The author has granted a nonexclusive license allowing Library and Archives Canada to reproduce, publish, archive, preserve, conserve, communicate to the public by telecommunication or on the Internet, loan, distribute and sell theses worldwide, for commercial or noncommercial purposes, in microform, paper, electronic and/or any other formats.

The author retains copyright ownership and moral rights in this thesis. Neither the thesis nor substantial extracts from it may be printed or otherwise reproduced without the author's permission.
AVIS:

L'auteur a accordé une licence non exclusive permettant à la Bibliothèque et Archives Canada de reproduire, publier, archiver, sauvegarder, conserver, transmettre au public par télécommunication ou par l'Internet, prêter, distribuer et vendre des thèses partout dans le monde, à des fins commerciales ou autres, sur support microforme, papier, électronique et/ou autres formats.

L'auteur conserve la propriété du droit d'auteur et des droits moraux qui protège cette thèse. $\mathrm{Ni}$ la thèse ni des extraits substantiels de celle-ci ne doivent être imprimés ou autrement reproduits sans son autorisation.
In compliance with the Canadian Privacy Act some supporting forms may have been removed from this thesis.

While these forms may be included in the document page count, their removal does not represent any loss of content from the thesis.
Conformément à la loi canadienne sur la protection de la vie privée, quelques formulaires secondaires ont été enlevés de cette thèse.

Bien que ces formulaires aient inclus dans la pagination, il n'y aura aucun contenu manquant.

\section{Canada}


The undersigned hereby recommend to

The Faculty of Graduate Studies and Research

acceptance of the thesis

\section{Learning a Test Oracle towards Automating \\ Image Segmentation Evaluation \\ Submitted by \\ Kambiz Frounchi}

In partial fulfillment of the requirements for the degree of

Master of Applied Science

Professor L. C. Briand (Co-Supervisor)

Professor Y. Labiche (Co-Supervisor)

Professor V. Aitken (Department Chair)

Carleton University

December 2008 


\begin{abstract}
Image segmentation is the act of extracting particular structures of interest from an image. A lot of time and effort is spent in order to evaluate image segmentation algorithms. If the image segmentation algorithm does not provide accurate enough results or in verification and validation terms fails, the technical expert needs to modify it and rerun the whole test suite to verify the revised image segmentation algorithm. This process is repeated as the image segmentation algorithm evolves to its final acceptable version where the test suite passes. This evaluation process is mostly done manually at the moment and is therefore very time consuming, requiring the presence of reliable experts. In this thesis, a solution is proposed that uses machine learning techniques to semiautomate this evaluation process. During the initial learning phase, the expert is required to evaluate segmentations manually. The similarity between the segmentations produced by the initial versions of the segmentation algorithm is found by applying a set of comparison measures to pairs of segmentations from the same subject. This information is used by different machine learning algorithms to devise a classifier that can classify a pair of segmentations as being diagnostically consistent or inconsistent. In a second phase, once a valid classifier is learnt, a segmentation produced by any new version of the image segmentation algorithm under test will be automatically deemed correct or incorrect depending on whether it is diagnostically consistent with the segmentations previously deemed correct. In this second phase, there is no need for any intervention from human experts. To demonstrate the performance of the approach, we have applied the solution to the evaluation of the left heart ventricle segmentation and have gotten very promising results. This solution also helps find the best performing machine learning techniques and the similarity measures with the most discriminating power in the context of the application.
\end{abstract}




\section{ACKNOWLEDGEMENTS}

I would like to thank Professors Briand and Labiche for their active support throughout this research work. This thesis was a joint collaboration between Carleton University and the software and medical imaging departments in Siemens Corporate Research. I would like to thank Leo Grady and Rajesh Subramanyan in Siemens Corporate Research for their help throughout my work. Last but not least I would like to thank my family and friends for their moral support. 


\section{TABLE OF CONTENTS}

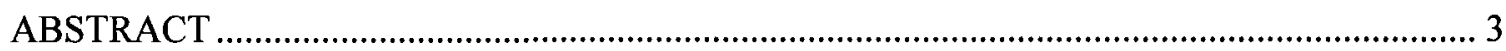

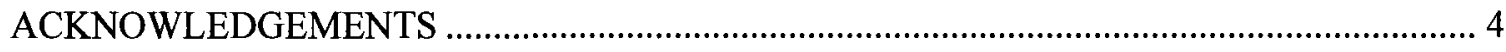

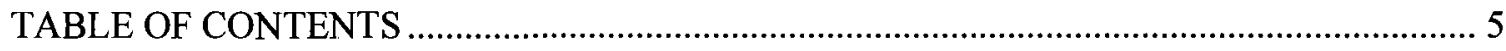

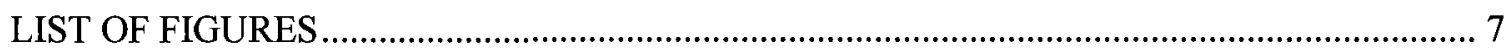

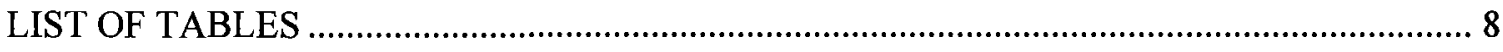

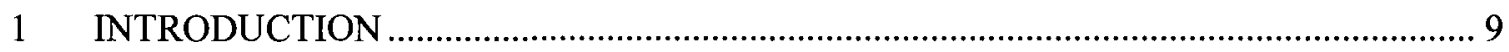

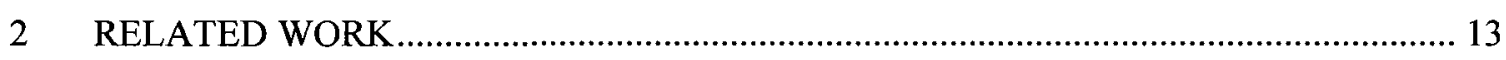

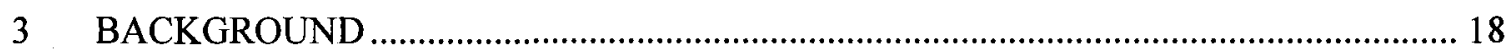

3.1 Image segmentation comparison techniques .......................................................... 18

3.1.1 Overlap measures ................................................................................ 20

3.1.2 Geometrical measures .............................................................................. 25

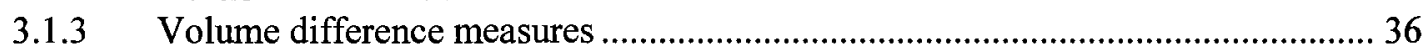

3.2 Machine learning techniques................................................................................. 36

4 IMAGE SEGMENTATION EVALUATION ORACLE DESIGN ………………………... 41

4.1 Segmentation evaluations swimlane....................................................................... 42

4.2 Learning classifier swimlane .............................................................................. 44

4.3 Tool support architecture...................................................................................... 46

5 CASE STUDY: LEFT HEART VENTRICLE SEGMENTATION EVALUATION........... 50

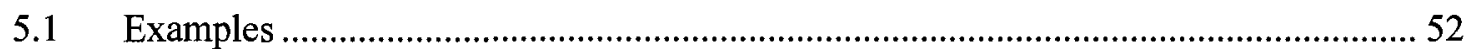

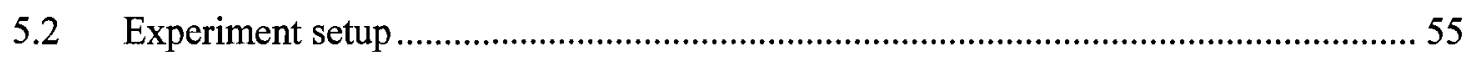

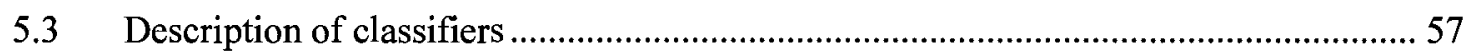

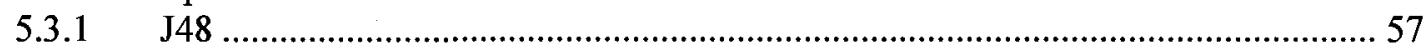

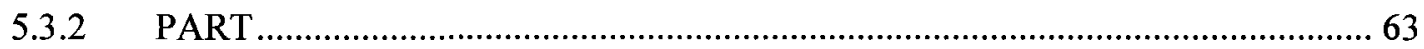

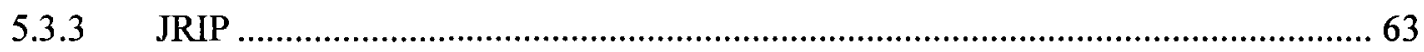

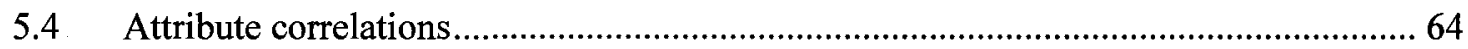

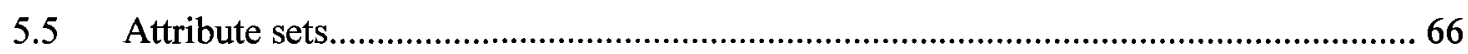

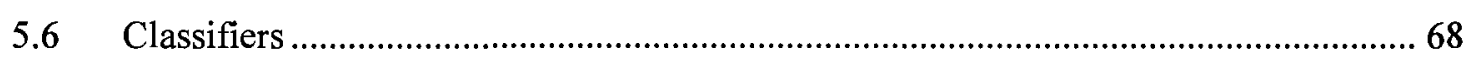

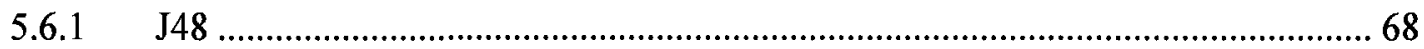

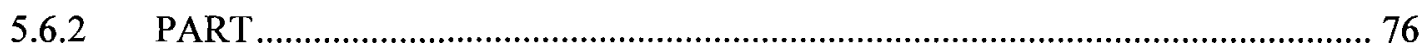

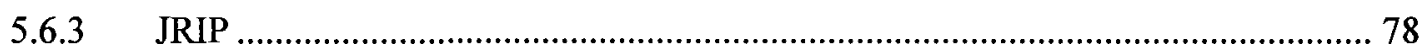

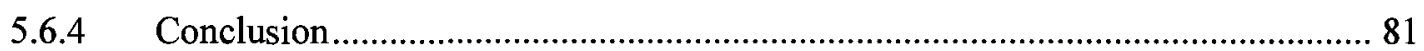

5.7 Classifier Performance Comparison ...................................................................... 83

5.7.1 Effect of using different measure types .......................................................... 85

5.7.2 Effect of using different data mining techniques.................................................... 86

5.7.3 Effect of using different attribute selection techniques ......................................... 87

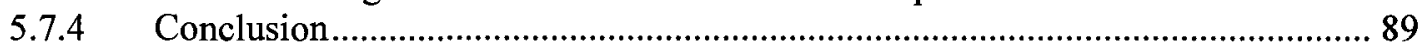

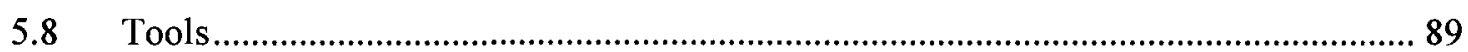

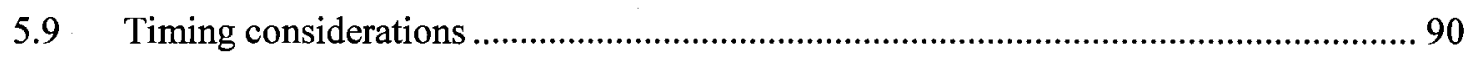

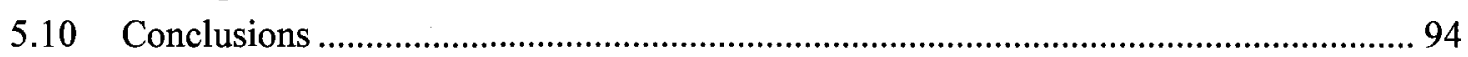




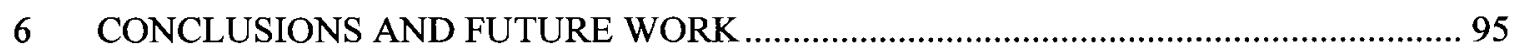

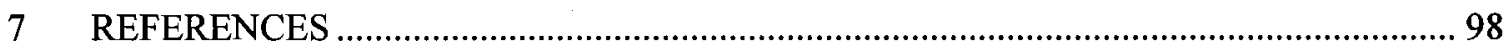




\section{LIST OF FIGURES}

Figure 1.1 - Manual Image Segmentation Evaluation Process................................................... 10

Figure 3.1 - Four mutually exclusive overlapping regions between the Segmentation Under Test

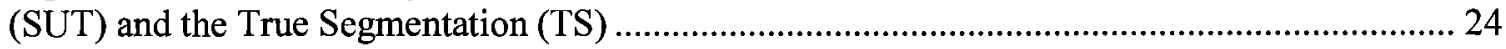

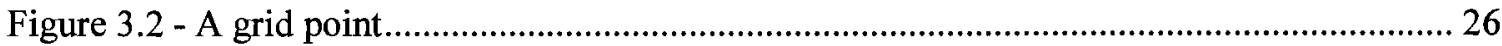

Figure 3.3 - Left: Segmentation; Center: $d_{4}$ distance transform; Right: $d_{8}$ distance transform

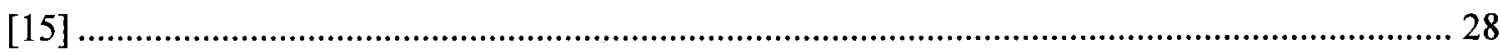

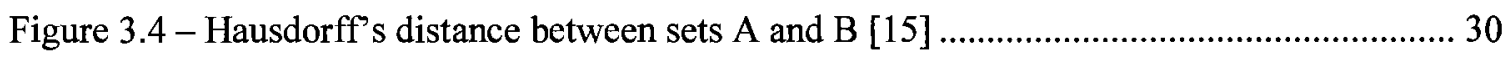

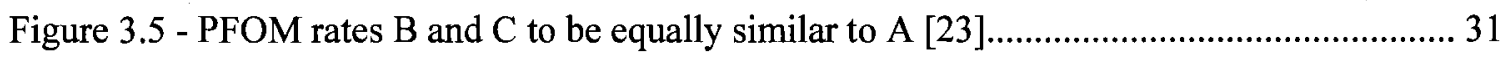

Figure 3.6 - Object is geometrically symmetrical around the principal axes $\left(x_{1}^{\prime}, x_{2}^{\prime}, x_{3}^{\prime}\right) \ldots \ldots \ldots . . .35$

Figure 4.1 - Semi-automated image segmentation algorithm evaluation process ....................... 41

Figure 4.2 - Structural components of the automated oracle .................................................. 47

Figure 5.1 - Correct Left Heart Ventricle Segmentation ....................................................... 53

Figure 5.2 - Incorrect Segmentation (oversegmentation to the left atrium) ............................... 53

Figure 5.3 - Incorrect Segmentation (Segmenting an incorrect region i.e. the right ventricle) .... 54

Figure 5.4 - Incorrect Segmentation (severe undersegmentation) ............................................. 54

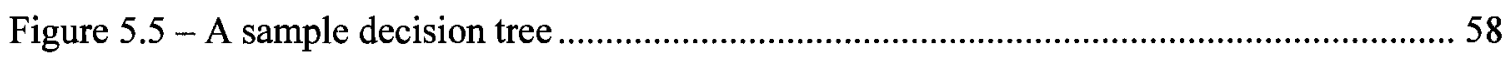

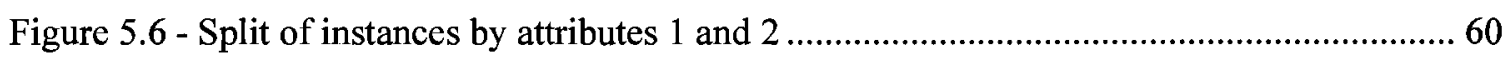

Figure 5.7 - (a) Top: J48 classifier trained with simple measures (OV) (b) Bottom: J48 classifier trained with the simple measures selected using J48 wrapper (OVW) ........................................ 71

Figure 5.8 - (a) Top: J48 classifier trained with geometrical measures (G) (b) Bottom: J48 classifier trained with the geometrical measures selected using J48 wrapper (GW)................... 73

Figure 5.9 - (a) Top: J48 classifier trained with all comparison measures (A) (b) Bottom: J48 classifier trained with the comparison measures selected using J48 wrapper (AW).................... 75

Figure 5.10 - Accuracy of J48, PART and JRIP classifiers versus each attribute set..................83

Figure 5.11 - Kappa Statistic of J48, PART and JRIP classifiers versus each attribute set......... 84

Figure 5.12 - Area under ROC curve of J48, PART and JRIP classifiers versus each attribute set

Figure 5.13 - Time consumption of comparison measures ( $\mathrm{x}$-axis = segmentation pair number, $\mathrm{y}$ -

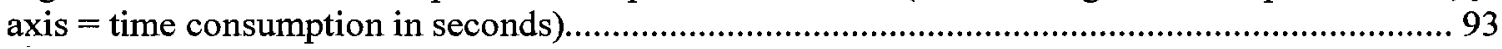




\section{LIST OF TABLES}

Table 3.1 - Two-class prediction outcomes.

Table 4.1 - Mapping between classifier results and the evaluation of the unknown image segmentation 43

Table 4.2 - Consistency of two segmentations 45

Table 4.3 - Table of all outputs produced by the previous versions of the SUT (version $<$ Vi) and their corresponding evaluations.

Table 4.4 - An example showing how the Consistency to Correctness Mapper module obtains the correctness of the OUT. 49

Table 5.1 - Details of case study. 55

Table 5.2 - Attributes used for machine learning 56

Table 5.3 - Spearman's rank correlation coefficient between highly correlated attribute pairs ... 65

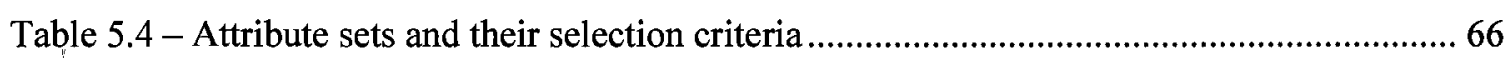

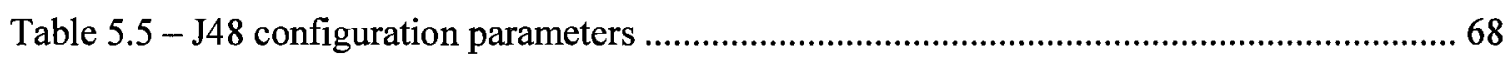

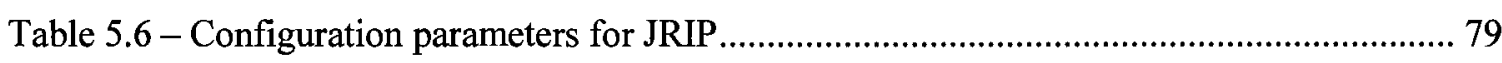

Table 5.7 - Comparison of classifier accuracies with respect to the attribute set category used for training (simple, geometrical, all) 86

Table 5.8 - Comparison of classifier accuracies with respect to the data mining technique (J48, PART, JRIP).

Table 5.9 - Comparison of classifier accuracies with respect to the use of filters and wrappers on the simple measures for training. 88

Table 5.10 - Comparison of classifier accuracies with respect to the use of filters and wrappers on the geometrical measures for training 88

Table 5.11 - Comparison of classifier accuracies with respect to the use of filters and wrappers on all of the measures for training. 88

Table 5.12 - Average comparison measure calculation times 94 


\section{INTRODUCTION}

Image segmentation is the act of extracting content from an image. Specifically, image segmentation refers to the act of delineating a particular object or structure of interest from an image [11]. Image segmentation algorithms have been devised to automatically segment an image without the need of an expert manually delineating the object of interest from the image. Experience has shown that devising an image segmentation algorithm is an iterative approach, as illustrated in Figure 1.1. The technical expert who devises each successive version of the image segmentation algorithm has to revise the initially devised image segmentation algorithm many times (steps 2, 3 and 4), verifying each revision manually (step 3) until the verification results indicate an acceptable revision of the image segmentation algorithm. To test each revision of the image segmentation algorithm, a sample set of subjects (or images) is used, a segmentation of each subject is obtained by applying the revision of the segmentation algorithm that is under test to each subject, and a few technical and medical experts verify the correctness of the produced image segmentations by grading them (step 3): Typically, a 1 to 5 scale is used. In software testing terms, the experts play the role of the (test) oracle that decides whether a test case (i.e., a subject in this case) passes or not (i.e., the subject segmentation is adequate). If all of the image segmentations receive a satisfactory grade by all of the experts, then the image segmentation algorithm is considered to be satisfactory. Other criteria may be considered such as enforcing that specific segmented subjects, that have particular diagnostically significant features, get a minimum acceptable grade for the image segmentation algorithm to be considered valid. Otherwise, the image segmentation algorithm has to be revised (step 4) and the same manual testing procedure has to be repeated for the newly revised image segmentation algorithm (steps 2 and 3).

This testing procedure is time-consuming and expensive as it requires the availability of medical experts to verify the segmentations. It may suffer from human errors made by the medical expert during the manual verification of a large number of images, and is also prone to different opinions by different medical experts (inter-expert variability) [12]. Providing semi-automated support for this manual verification and validation process will 
partially solve the problems above and lead to (1) the improvement of the image segmentation algorithms and (2) creating a satisfactory image segmentation algorithm in less time and therefore at lower cost, since part of the time that was devoted to manually verifying the segmentations will now be put on improving the quality of the image segmentation algorithm.

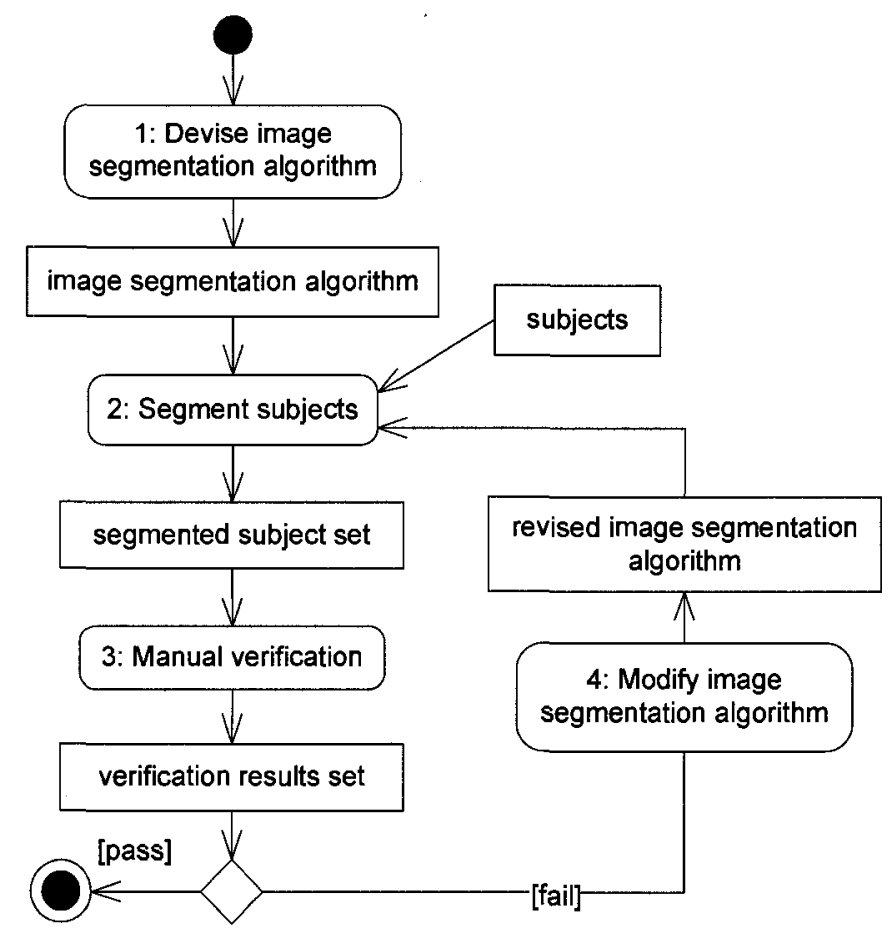

\section{Figure 1.1 - Manual Image Segmentation Evaluation Process}

One way to verify if a newly generated (by a new revision of the algorithm) image segmentation is correct is to compare it with a segmentation of the same subject produced by a previous revision of the algorithm that was deemed to be correct by technical and medical experts. A newly generated segmentation deemed "similar", thanks to one or several measures, to a segmentation previously graded as correct would then be considered correct too. This raises two questions: Which measures should be used to compare two segmentations? What does it mean for a segmentation to be "similar" to another one for the same subject?

It is not realistic to compare segmentations pixels by pixels (or voxels in a $3 \mathrm{D}$ image) as two segmentations of the same subject could slightly differ, pixel/voxel-wise, and still be 
equivalent from the point of view of the expert. Instead, there exist a number of measures, currently used and empirically validated in the image processing domain that can be used to compare segmentations.

One way to use such similarity measures to compare segmentations is to set a threshold and argue that if a new segmentation diverges from a segmentation previously characterized as correct by more than the threshold value, then the new segmentation is not correct. However, we would need to define a threshold beforehand, which could only be highly subjective and unlikely to be optimal. Instead, we intend to rely on a machine learning algorithm to develop decision models (classifiers). This decision model would characterize which similarity measures and which deviations of similarity measure values between new segmentations and previous segmentations (deemed to be correct) are indicative of (in)correct segmentations. In this way while one measure may not be a good indicator of the similarity between a pair of segmentations, but many such measures, each good at reflecting some aspect of the similarity between the two segmentations, can be combined by the machine learning algorithm to indicate the consistency of the segmentation pair with high accuracy.

As a result, once we have learnt a sufficiently accurate decision mode (classifier), testing the image segmentations (step 3 in Figure 1.1) will no longer require human intervention. In other words, human expert knowledge will only be required to learn the decision model, which will then be used to support automated decisions. Therefore, the whole image segmentation algorithm testing process will be semi-automated. This will mean fewer opportunities for medical expert errors, no inter-expert variability, less time spent by the experts to verify the different revisions of the image segmentation algorithm, and as mentioned before more time for improving the quality of the image segmentation algorithm.

In a nutshell the main contributions of this thesis are (1) devising a re-usable semiautomated approach that attempts to solve the oracle problem for testing (medical) image segmentation algorithms. In doing so, the approach (2) helps to find the appropriate 
machine learning techniques and (3) similarity measures pertinent to the application. Last, the approach is applied on the problem of left heart ventricle segmentations.

The remainder of this thesis is structured as follows. It starts with a discussion of related work on the oracle problem (Chapter 2). Chapter 3 gives some background on image segmentation comparison techniques and machine learning methods. Chapter 4 explains our generic approach towards the semi-automated evaluation of image segmentation algorithms. The results obtained in the case study (left heart ventricle segmentation algorithm verification) are explained in Chapter 5. Chapter 6 concludes the thesis with a discussion of future steps. 


\section{RELATED WORK}

There is a large body of work in the image processing field on studying and improving image segmentation algorithms. This body of work is however out of the scope of this thesis and is therefore not further discussed here.

An oracle is a procedure that assesses whether the observed behavior of the software under test matches its expected behavior. The oracle will output either a "yes" or "no" and it may include an explanation as to why this decision was made [4]. Whether we can define such a procedure to assess the correctness of the software under test is referred to as the oracle problem.

Defining an oracle is not a trivial task and it may not be feasible to define one at all times. Even if it is theoretically possible to define an oracle, it may not be practically possible to verify the correctness of the software under test in a reasonable amount of time while putting a reasonable amount of effort [2]. In [1], software that we cannot devise an oracle for are referred to as un-testable software and lie into three categories:

1) Software where the output is unknown. An example for this situation is given in [3]; consider a program that analyzes petri-nets and outputs the probability of being in a particular state; It is very difficult to verify whether all the digits of the output probability are correct or not.

2) Software where the output is of a very large volume and thus cannot be verified in a reasonable amount of time.

3) Software where the tester has a misconception about its correct behavior. This is usually the case where the oracle is based on a different specification than the specification that the implementation of the software under test is based on. 
Different solutions have been proposed to the oracle problem. One approach is design redundancy $[1,[2]$, where different implementations of the same specification are produced. If the outputs produced by two different implementations on the same input differ, we can conclude that there is a problem: either one of the two versions has a problem, or the two versions both have a problem. When the outputs are the same, we may express some confidence that the software under test is behaving correctly, but this may still not be a valid assumption as both programs may be producing similar incorrect results. However, if the two versions were independently designed, this should be unlikely. The assumption of independence in multi-version programming has been explored in [8]. The results of this empirical study show that the independence assumption in multi-version programming may not hold at all times and care should be taken on the reliability of multi-version programming. More than two independently designed versions can also be used: a consensus strategy has to be adopted where if the outputs from a large number of the implementations agree with the output of the software under test, the software is deemed to have produced the correct output. Implementing multiple versions of the same specification of the software under test is a time-consuming and expensive activity. The effort going into implementing the extra versions may be as much as implementing the software under test and the agreement of the outputs of the software under test and the other version(s) does not necessarily imply that the output of the software under test is correct. It is proposed in [1] that high level languages should be used for the implementation of the extra versions, but this may not be a feasible choice at all times. For example, using high level languages for the implementation of highly memory-intensive and cpu-intensive applications would result in a very high time overhead for obtaining the results of these slow programs and thus immensely increase the time required for testing the software under test. However, some researchers have argued that multi-version programming does not necessarily add a significant amount to the cost since the implementation of the software is just a minor part of the overall software development process [10]. The notion of multi versions of a program is also used in regression testing [3]. In this context, previous versions of the program can be used as references of correct behavior as the software evolves. 
Another approach to the oracle problem is data redundancy. In this approach, the output of the software under test is verified by testing how it behaves with respect to other inputs. For example, consider an algorithm to calculate the $\sin (\mathrm{x})$ for some input $\mathrm{x}$. Using the identity $\sin (a+b)=\sin (a) \sin \left(\frac{\pi}{2}-b\right)+\sin \left(\frac{\pi}{2}-a\right) \sin (b)$, if $(\mathrm{a}+\mathrm{b}=\mathrm{x}), \sin (\mathrm{x})$ can be verified by changing the value of $a$ and $b$ [3]. This approach works very well when such mathematical identities can be found in the particular application. Other examples of the use of this technique is when we know that the output of the software under test for a given input data should vary slightly if we feed it with data that is very close to the input data. Large variations would indicate to the oracle that the software under test is not behaving properly. $N$-copy programming [6] is also a data redundancy technique. Although introduced in the context of fault tolerance the principle can be used in testing. In $\mathrm{N}$-copy programming, a re-expression algorithm is used to re-express the input data in $\mathrm{N}$ different forms (i.e. make $\mathrm{N}$ copies of the data) which are all logically equivalent to the input data. The outputs of executing the program on the $\mathrm{N}$ different copies are fed into a voter which can be thought of as the oracle in a testing scenario. If a majority of the $\mathrm{N}$ outputs are very different from the output produced by the input data we can conclude that the software under test is behaving incorrectly. In the case where the majority agrees, we gain more confidence that the software is acting correctly. Using data redundancy, there is no overhead of implementing extra versions of the software under test which makes the data redundancy method cheaper than design redundancy, but the challenge is to find appropriate input/output relationships.

Another alternative to design and data redundancy is consistency checks. In this approach the oracle checks if the outputs of the software under test are consistent, i.e., whether they conform to some known facts. For example in a program that outputs probabilities, one consistency check is checking that the output probability cannot exceed one or be negative. An alternative is to narrow down the output domain to a plausible output domain and check whether the consistency check is valid or not for those outputs when executing the test cases [1]. This kind of checking cannot prove that the software under test is correct but it can help find faults in the software. 
Another alternative towards tackling the oracle problem is to only test the software under test on simplified data, i.e., the data that the output is known for [2]. The obvious drawback to this approach is that the software under test usually has faulty behavior in the more complicated scenarios.

In the case where formal specifications exist for the software under test, researchers have proposed methods to derive oracles from the formal specifications. This has been investigated for real-time systems in [7], where the test class consists of some test executions or sequences of stimuli. Test oracles in the form of assertions are obtained using symbolic interpretation of the specifications for each control point. A discussion on oracles that are composed of assertions based on properties specified either in first order logic or computation tree logic is given in [5]. Assertions under the form of pre and postconditions can also be used as test oracles [9].

In [19], machine learning is used to evaluate which of the two segmentations produced by different segmentation algorithms or with different parameterizations of the same segmentation algorithm is better in delineating the objects of interest. In their study they combine the evaluations made by standalone methods to make this decision. Standalone methods evaluate the goodness of a segmentation by applying some quantitative measure to the segmentation. The quantities measured may be for example the color uniformity of the labeled region, which requires information from the original image in addition to just the labeling from the segmentation. Decision trees are used to model these standalone methods though how the decision trees are constructed and how accurately they model the standalone methods is not described accurately. The evaluations made by each of the decision trees are combined using meta-learning, which essentially combines the decisions of the different decision trees, each predicting which of the two segmentations are better, and makes a final decision. A fundamental difference in the use of machine learning in their research work and ours is that we try to use machine learning to find the best subset of comparison measures between two segmentations that could predict the consistency of two segmentations while in [19] they use standalone methods which are applied to only one segmentation each time. Also, the similarity measures that we define do not require any information from the original images (such as information regarding 
color, texture, etc.), only using information from the segmentations (the extent and shape of labeling) to get a measure of consistency, while the standalone methods in [19] generally do. 


\section{BACKGROUND}

In this Chapter some background information will be given on two subjects of interest in this research work:

1. Image segmentation comparison techniques: As mentioned in the Introduction, we propose to study the similarities between segmentations with a number of measures. A discussion of some popular similarity measures that are used for comparing two segmentations is given in Section 3.1.

2. Machine learning techniques: As mentioned in the Introduction, we intend to rely on a machine learning algorithm to identify what it means for two segmentations to be similar. Different machine learning techniques will be evaluated to find an appropriate one in our context. A very brief discussion of the available machine learning techniques and the methods that are used to evaluate them is given in Section 3.2 .

\subsection{Image segmentation comparison techniques}

Image segmentation is the act of extracting content from an image. Specifically, image segmentation refers to the act of delineating a particular object or structure of interest from an image [11]. Image segmentation algorithms have been devised to automatically segment an image without the need of an expert manually delineating the object of interest from the image. These image segmentation algorithms have to be verified and validated. This can be done by evaluating the correctness of the segmented images. Some methods that are used to evaluate the correctness of the segmented images, in the medical domain are:

- Manual evaluation: The segmentation is compared with the manual segmentation produced by a medical expert, or directly evaluated by the medical expert.

- Physical and Computational Phantoms: Phantoms are either physical or computational models that are obtained from a known true segmentation for 
evaluating image segmentation algorithms [12]. The segmentation obtained from applying the segmentation algorithm to a physical or computational phantom is compared with the known true segmentation that the physical or computational phantom is constructed for.

Manual evaluation suffers from the following obstacles [12]:

- The time, effort, and cost entailed when relying on medical experts who are scarce resources.

- Medical expert error: The medical expert may wrongly segment the image due to poor vision or factors related to the quality and resolution of the image.

- Inter-expert variability: Different experts may segment the image differently, causing confusion in finding the surrogate of truth.

Phantoms also have disadvantages. Physical phantoms usually do not depict a realistic anatomical model and digital phantoms use a very simplistic model of the image acquisition process [16].

In summary, in order to evaluate the correctness of an image segmentation algorithm, generated segmentations have to be compared with some surrogate of truth. This surrogate may be the manual delineation of the segmentation obtained from a medical expert, the true segmentation that the phantom is constructed for, or a different segmentation algorithm (version) that was assessed to be correct by a human expert. This last form is more relevant to the context of this research work since the segmentation algorithm development process is iterative: during iteration $i$, i.e., when building version $i$ of the segmentation algorithm, segmentations produced during the evaluation of iteration $j(j<i)$, i.e., when building version $j$ of the segmentation algorithm, that were assessed as correct can be used to check version $i$. When comparing segmentations produced by versions $i$ and $j$ of the segmentation algorithm, three families of measures can be considered: measures that simply look at the extent of overlapping of the two segmentations (Section 3.1.1), measures that compare the shapes of the two 
segmentations, referred here to as geometrical measures (Section 3.1.2), and measures that compare the volumes delineated by the two segmentations (Section 3.1.3).

A 2D digital picture is a grid of a finite number of pixels. Each pixel is denoted with its location and a value at that location [15]. In the case of 3D digital images, the term voxel is used instead of pixel, but in this text pixel refers interchangeably to both $2 \mathrm{D}$ and $3 \mathrm{D}$ cases. The value associated with each pixel location could be the color of that location or any other relevant value. In this text, the segmentation is considered as a digital picture and the value associated with each pixel is its label in the segmentation.

\subsubsection{Overlap measures}

A label is an identifier of a particular structure of interest in an image. As a result of segmenting an image, each point is associated with one to many labels. If each point is associated with only one label, the segmentation is referred to as a hard segmentation. Otherwise the segmentation is said to be fuzzy. Different overlapping measures can be devised for these two kinds of segmentations.

\subsubsection{Measures for hard segmentations}

To compare hard segmentations, a number of similarity measures have been defined. The main ones calculate the amount of overlap of the labeled regions between two segmentations (usually the segmentation under test and the segmentation that is considered to be the surrogate of truth):

- Dice Similarity Coefficient (DSC) [14]:

Equation 3.1 $\quad D S C=\frac{2 N\left(L R_{\text {sut }} \cap L R_{t s}\right)}{N\left(L R_{\text {sut }}\right)+N\left(L R_{t s}\right)}$

where $L R_{s u t}$ is the labeled region in the Segmentation Under Test (SUT), $L R_{t s}$ is the labeled region in the True Segmentation (TS), and $N(L R)$ is the number of pixels in the labeled region LR. 
- Tanimoto Coefficient (TC) [2]:

Equation 3.2 $T C=\frac{N\left(L R_{\text {sut }} \cap L R_{t s}\right)}{N\left(L R_{\text {sut }} \cup L R_{t s}\right)}$

The DSC and the TC coefficients have the same numerator which is the number of pixels that overlap among the common labeled region of the segmentation under test and the true segmentation. This number is divided by the total number of pixels in the two labeled regions in DSC, and by the number of pixels in the union of the two labeled regions in TC. Both of these measures are simply trying to measure the percentage of common label between the segmentation under test and the true segmentation.

In the above two coefficients, each pixel in a segmentation is either in a labeled region or is not, and no two labeled regions overlap in any one (hard) segmentation. In the simplest case we only have two labels in a segmentation, one representing the regions where the structure of interest is located and one representing the rest of the segmentation. The notation in Equation 3.1 and Equation 3.2 assume there is only one region of interest (which will be the case in our case studies). The notation can easily be extended to account for more than two labels (e.g., by subscripting LR with the label name).

\subsubsection{Measures for fuzzy segmentations}

In fuzzy segmentations, each pixel may be a part of more than one label. The percentage of involvement of each pixel $p$ in each labeled region $l$ in $S$ (the set of all the pixels in the segmentation) can be represented by the membership function $m_{S, l}(p) \in[0,1], m_{S}(p)=\sum_{l \in \text { Labels }} m_{S, l}(p)=1$. Fuzzy segmentations are a better representation of reality as different labels may overlap in medical images due to partialvolume effects. Partial volume effects are caused when multiple tissues contribute to the same pixel causing the blurring of the medical image in those set of pixels [4].

Fuzzy segmentations can be compared using fuzzy measures. Before defining the fuzzy measures in more detail, we first provide some fuzzy set theory definitions: Equation 3.3 to Equation 3.7. In these definitions, $A$ and $B$ are two sets of pixels. 
The membership functions for each pixel $p$ in the intersection $(A \cap B)$, union $(A \cup B)$ and difference ( $A-B$ ) of the sets $A$ and $B$ are defined in Equation 3.3, Equation 3.4, and Equation 3.6.

Equation 3.3 $\quad m_{A \cap B}(p)=\sum_{l \in \text { Labels }} \alpha_{l}\left(\operatorname{MIN}\left(m_{A, l}(p), m_{B, l}(p)\right)\right)$

Equation 3.4 $\quad m_{A \cup B}(p)=\sum_{l \in \text { Labels }} \alpha_{l} \sum_{p \in P \text { Pixels }} M A X\left(m_{A, l}(p), m_{B, l}(p)\right)$

Equation 3.5 $\quad m_{A-B, l}(p)=\left\{\begin{array}{l}m_{A, l}(p)-m_{B, l}(p) \quad m_{A, l}(p)-m_{B, l}(p) \geq 0 \\ 0 \quad \text { otherwise }\end{array}\right.$

Equation 3.6 $\quad m_{A-B}(p)=\sum_{l \in \text { Labels }} \alpha_{l} \sum_{p \in \text { Pixels }} m_{A-B, l}(p)$

The cardinality for set $A$ is defined in Equation 3.7.

Equation $3.7 \quad|A|=\sum_{l \in L a b e l s} \alpha_{l} \sum_{p \in \text { Pixels }}\left(m_{A, l}(p)\right)$

In Equation 3.3 and Equation 3.4, $M I N\left(m_{A, l}(p), m_{B, l}(p)\right)$ is the minimum of the assigned membership of label $l$ to pixel $p$ in the sets $A$ and $B, M A X\left(m_{A, l}(p), m_{B, l}(p)\right)$ is the maximum of the assigned membership of label $l$ to pixel $p$ in the sets $A$ and $B$. In Equation 3.3 to Equation 3.7, $\alpha_{l} \in[0,1], \sum_{l \in \text { Labels }} \alpha_{l}=1$, where $\alpha_{l}$ is the weight of each label. Assigning different weights to different labels is useful when some labels are of more importance than others.

A fuzzy overlap definition for comparing two segmentations $\left(\mathrm{TC}_{\mathrm{MF}}\right)$ is defined ${ }^{1}$ in Equation 3.8, where SUT and TS refer to the set of pixels that are respectively in the segmentation under test and the true segmentation.

\footnotetext{
${ }^{1}$ This definition is similar to equation (3) in [14], but described in a different way.
} 
Equation 3.8 $T C_{M F}=\frac{\sum_{p \in P \text { Pixels }} m_{S U T \cap T S}(p)}{\sum_{p \in P \text { Pixels }} m_{\text {SUT } \cup T S}(p)}$

Equation 3.8 is a good overlap comparison measure when the image segmentation algorithm produces a multi-label fuzzy segmentation, but fuzzy segmentations are a generalization of hard segmentations as are fuzzy measures a generalization of non-fuzzy measures, so Equation 3.8 can be used for hard segmentations too. In $\mathrm{TC}_{\mathrm{MF}}$, a value of zero would indicate zero overlap between the segmentation under test and the true segmentation while a value of 1 indicates 100 percent overlap between them.

\subsubsection{Statistical decision theory measures}

Some measures derived from statistical decision theory have been suggested for comparing segmentations [12]. The measures are based on the definition of four mutually exclusive regions in Figure 3.1 for a 2-D situation, referred to as TP (True Positive), TN (True Negative), FP (False Positive) and FN (False Negative). In the definition of TN, U is the union of the four mutually exclusive regions: $U=T P \cup T N \cup F P \cup F N$.

TP is the Truly Positive area, i.e., the intersection of the segmentation under test and the true segmentation: $T P=S U T \cap T S$. TN is the Truly Negative area, i.e., the area that does not intersect with neither the true segmentation nor the segmentation under test: $T N=U-S U T-T S$. FP is the Falsely Positive area, i.e., the area in the segmentation under test that does not intersect with the true segmentation: $F P=S U T-T S$. FN is the Falsely Negative area, i.e., the area in the true segmentation that does not intersect with the segmentation under test: $F N=T S-S U T$. 


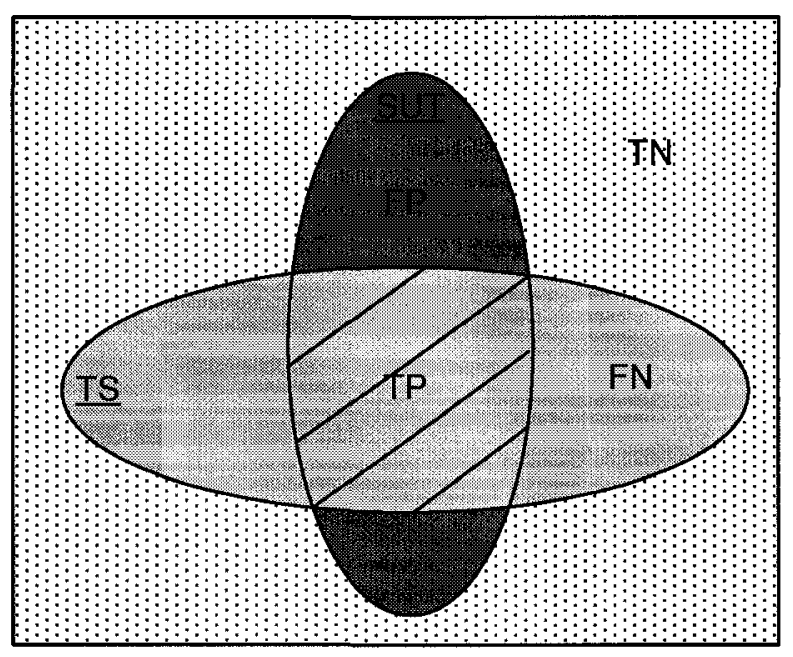

Figure 3.1 - Four mutually exclusive overlapping regions between the Segmentation Under Test (SUT) and the True Segmentation (TS)

Using the above definitions the two measures Truly Positive Volume Fraction (TPVF) and Falsely Positive Volume Fraction (FPVF) are defined in Equation 3.9 and Equation 3.10 .

Equation 3.9 $T P V F=\frac{\sum_{p \in \text { Pixels }} m_{T P}(p)}{\sum_{p \in \text { Pixels }} m_{T S}(p)}$

Equation 3.10 $F P V F=\frac{\sum_{p \in \text { Pixels }} m_{F P}(p)}{\sum_{p \in \text { Pixels }} m_{U_{N}}(p)}$

In Equation 3.10, $U_{N}=U-T S$ and represents the total negative region or the region that does not intersect with the true segmentation. These measures have been defined using fuzzy notation for generalization purposes. TPVF divides the truly positive region by the true segmentation region and FPVF divides the falsely positive region by the total negative region. It is desirable for TPVF and (1-FPVF) to both be close to 1 in order to have a significant overlap between the two segmentations being compared, thus leading to a segmentation that is accurate enough to support medical diagnostic. FPVF can detect 
the situations where the segmentation under test has a high percentage of overlap with the correct segmentation but incorrectly labels other regions too.

\subsubsection{Geometrical measures}

The measures defined in Section 3.1.1 are all overlap measures calculating the amount of overlap between the two segmentations under comparison. Geometrical measures go beyond calculating the overlap. They take into account the differences in the shapes of the two segmentations under comparison, capturing differences such as the size and position of the segmentation boundaries. Overlap measures may be sufficient in many cases to detect severe oversegmentation (segmentation under test labeling pixels that are not of interest) or severe undersegmentation (segmentation under test not labeling pixels that are of interest) but they do not perform well in situations where the segmentation under test has a reasonably high percentage of overlap with the correct segmentation, but incorrectly labels some pixels of interest that make the segmentation diagnostically unacceptable from the standpoint of a medical expert and thus the segmentation has to be evaluated as incorrect. In these cases, geometrical measures may help capture the subtle differences between the segmentation under test and the correct segmentation. We present below the geometrical measures that are used in this research work.

\subsubsection{Average Distance to Boundary Difference}

Let $S$ be any arbitrary set of pixels. $d: S \times S \mapsto R$ is a metric or distance function if it satisfies the following condition [15]:

Equation 3.11 $0=d(p, p) \leq d(p, q)+d(q, p)=2 d(p, q)$ for all $p, q \in S$

[S,d] defines a metric space. A widely used metric is the Euclidean metric.

Using an n-dimensional Cartesian coordinates system, let $p=\left(x_{1}, x_{2}, \ldots, x_{n}\right)$ and $q=\left(y_{1}\right.$, $\left.y_{2}, \ldots, y_{n}\right)$ be any two pixels in $\mathrm{R}^{\mathrm{n}}(\mathrm{n} \geq 1)$. The Euclidean metric $d_{e}$ is defined as follows [15]:

Equation 3.12 $\quad d_{e}(p, q)=\sqrt{\left(x_{1}-y_{1}\right)^{2}+\cdots+\left(x_{n}-y_{n}\right)^{2}}$ 
Some other widely used metrics are the city-block or Manhattan metric (Equation 3.13) and the chessboard metric (Equation 3.14) [15].

Equation 3.13 $\quad d_{4}(p, q)=\left|x_{1}-x_{2}\right|+\left|y_{1}-y_{2}\right|$

Equation 3.14 $d_{8}(p, q)=\max \left\{\left|x_{1}-x_{2}\right|,\left|y_{1}-y_{2}\right|\right\}$

If $p$ and $q$ are restricted to $Z^{2}\left(Z\right.$ is the set of integers), $d_{4}(p, q)$ is the number of horizontal or vertical unit-length steps (where only one coordinate changes) from $p$ to $q . d_{8}(p, q)$ can also take diagonal moves where both coordinates change similar to the moves of a king in chess (notice the resemblance between the names of these metrics and their definition).

To better visualize the city-block and chessboard metrics we can see from Figure 3.2 that $\mathrm{d}_{4}(\mathrm{p}, \mathrm{q})=3, \mathrm{~d}_{4}(\mathrm{p}, \mathrm{r})=6$ while $\mathrm{d}_{8}(\mathrm{p}, \mathrm{q})=2, \mathrm{~d}_{8}(\mathrm{p}, \mathrm{r})=4$.

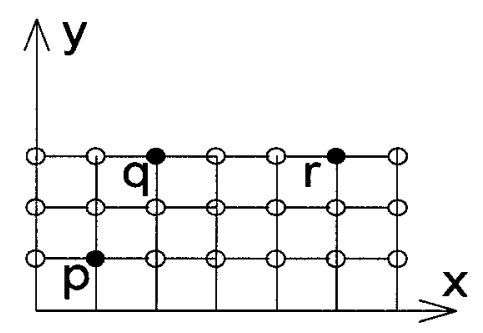

Figure 3.2 - A grid point

The reason for the $d_{4}$ and $d_{8}$ notation used in the city-block and chessboard metric definitions is that each pixel has respectively 4 or 8 adjacent pixels (neighbors) where the neighbors of the pixel $p$ are defined in Equation 3.15:

Equation 3.15 $N_{\alpha}(p)=\left\{q \in Z^{2}: d_{\alpha}(p, q) \leq 1\right\}$

where $N_{\alpha}(p)$ is the set of neighbor pixels of pixel $p . \alpha$ is the number of neighbor pixels.

We can extend the chessboard metric to 3D (Equation 3.16) in which case each pixel has 26 neighbors. 
Equation 3.16

$$
d_{26}(p, q)=\max \left\{\left|x_{1}-x_{2}\right|,\left|y_{1}-y_{2}\right|,\left|z_{1}-z_{2}\right|\right\}
$$

If $S$ represents the image, $\langle S>$ the segmentation, i.e. the set of labeled pixels in the image, and $<\bar{S}>$ the set of non-labeled pixels in the image, for any metric $d$ the $d$ distance transform of $S$ associates with each pixel $p$ of $\langle S\rangle$ the distance to $\langle\bar{S}\rangle$. This distance is the minimum distance of pixel $p$ to the boundary of $\langle\bar{S}>$ and to find it we can scan the adjacent neighbor pixels to $p$, finding the closest one to the boundary and assigning $p$ the distance of the closest neighbor to the boundary plus the distance between pixel $p$ and that neighbor pixel.

In order to bring more intuition on how the distance map is calculated, we describe a two pass algorithm to calculate the $d_{\alpha}(\alpha \in\{4,8\})$ distance transform in the 2D context [15] though a similar approach can be used in the 3D case:

1) Pass 1: scan $\mathrm{S}$ from left-to-right, top-to-bottom and calculate $f_{1}(p)$ as follows:

Equation 3.17 $f_{1}(p)=\left\{\begin{array}{cc}0 & \text { if } p \in<\bar{S}> \\ \min \left\{f_{1}(q)+1: q \in B(p)\right\} & \text { if } p \in<S>\end{array}\right.$ $B(p)$ for pixel $p$ with coordinates $(x, y)$, is the two pixels $(x, y+1)$ and $(x-1, y)$ if the $d_{4}$ distance transform is used. In case of using the $d_{8}$ distance transform the neighbor pixels with coordinates $(x-1, y+1)$ and $(x+1, y+1)$ are also considered.

2) Pass 2: scan $\mathrm{S}$ from right-to-left, bottom-to-top and calculate $f_{2}(p)$ as follows.

Equation 3.18 $f_{2}(p)=\min \left\{f_{1}(p), f_{2}(q)+1: q \in A(p)\right\}$

$A(p)$ for pixel $p$ with coordinates $(x, y)$, is the two pixels $(x, y-1)$ and $(x+1, y)$ if the $d_{4}$ distance transform is used. In case of using the $d_{8}$ distance transform the neighbor pixels with coordinates $(x+1, y-1)$ and $(x-1, y-1)$ are also considered.

The distance to the boundary of the segmentation for each pixel $p$ in the segmentation $S$ can be calculated in Equation 3.19: 


\section{Equation 3.19 $\quad d_{\alpha}(p,<\bar{S}>)=f_{2}(p)$ for all $p$ in $S$}

The $d_{4}$ and $d_{8}$ distance transforms are shown in Figure 3.3 for an arbitrary 2D segmentation. The difference between the two right figures is that with the $d_{8}$ distance transform, moves on the diagonals are allowed when computing the distance, resulting in smaller (and more close to the Euclidean distance) values of the distances than with $d_{4}$, thus obtaining a more accurate distance map of the segmentation. As in the 3D case each pixel has 26 neighbors, the $d_{26}$ distance transform can be used in the 3D case, where 26 neighbors of each pixel in the 3D segmentation are scanned.
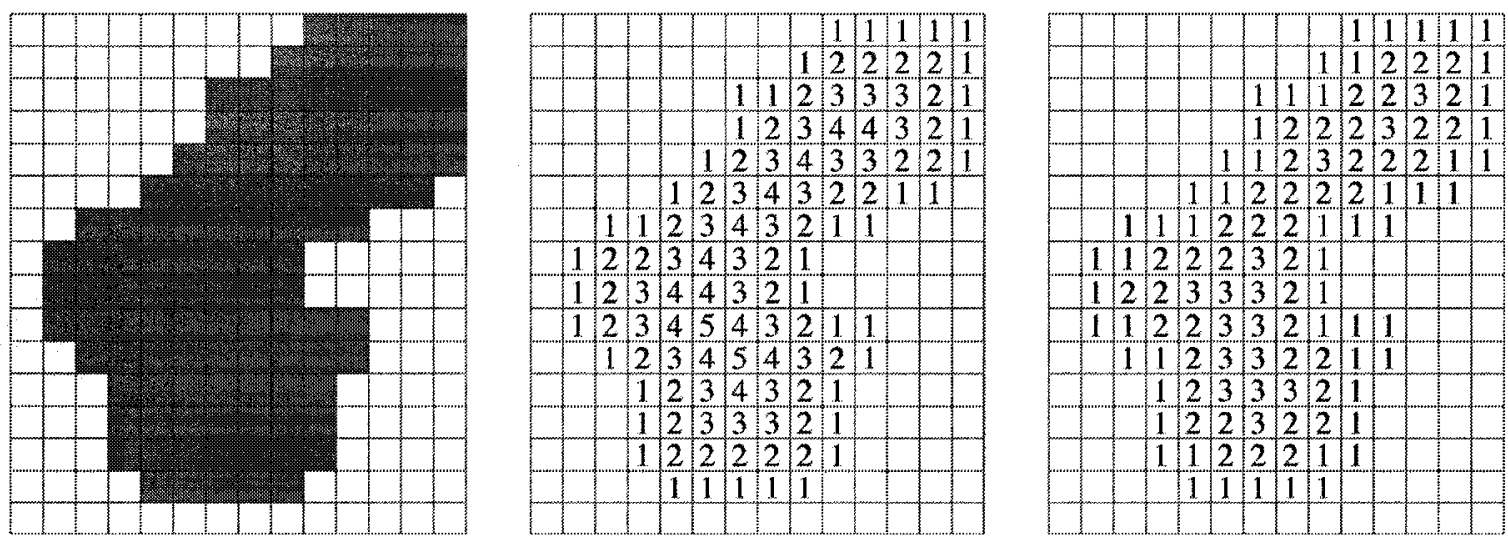

Figure 3.3 - Left: Segmentation; Center: $d_{4}$ distance transform; Right: $d_{8}$ distance

\section{transform [15]}

The calculation of the $d_{e}$ distance transform is somewhat more complicated and will not be discussed here. Regardless of the metric used to calculate the distance transform (i.e. city-block, chessboard or Euclidean for example) we can calculate the Average Distance to the Boundary (ADB) of each segmentation (Segmentation Under Test and the True Segmentation) over all the pixels in the segmentation (Equation 3.20) and compare the segmentations by measuring the difference between these average distances (Equation 3.21). ADBD stands for Average Distance to Boundary Difference. In Equation 3.20, $d(p,<\bar{S}>)$ represents the distance from pixel $\mathrm{p}$ to the boundary $(<\bar{S}>)$ that is assigned by the distance map. 
Equation 3.20 $\quad A D B=\frac{\sum_{p \in<S>} d(p,<\bar{S}>)}{\text { number of pixels in }<S>}$

Equation 3.21 $A D B D=\left|A D B_{t s}-A D B_{\text {sut }}\right|$

\subsubsection{Hausdorff's Distance}

Hausdorff's distance calculates the distance between two sets of pixels [15]:

Equation 3.22 $H D(A, B)=\max \left\{\max _{p \in A} \min _{q \in B} d(p, q), \max _{p \in B} \min _{q \in A} d(p, q)\right\}$

In Equation 3.22 $\mathrm{A}$ and $\mathrm{B}$ are two sets of pixels. $d$ can be any metric such as the Euclidean metric (as shown in Figure 3.4) or the chamfer metric. Effectively, this equation means that each pixel in each set is scanned to find the minimum distance between that pixel and the boundary of the other set; for each set, the pixel that is farthest to the boundary of the other set is chosen, and the distance between the two sets is the larger distance of the two chosen pixels in each set. Figure 3.4 shows the Hausdorff distance between the sets $\mathrm{A}$ and $\mathrm{B}$ in the Euclidean space. In Figure 3.4, $\max _{p \in A} \min _{q \in B} d(p, q)$ is the length of the arrow pointing from pixel $p, \max _{p \in B} \min _{q \in A} d(p, q)$ is the length of the arrow pointing from pixel $q$ and the maximum of these two lengths is the distance between sets A and B (i.e., the length of the arrow pointing from pixel $p$ ). The Hausdorff measure is noise sensitive and a single pixel that is incorrectly part of any of the sets could have a great influence on the value of this measure [16]. In the context of this text, the two sets of interest are the set of labeled pixels in the segmentation under test and the set of labeled pixels in the true segmentation. Hausdorff's distance could range from 0 to infinity as it is not a bounded measure. 


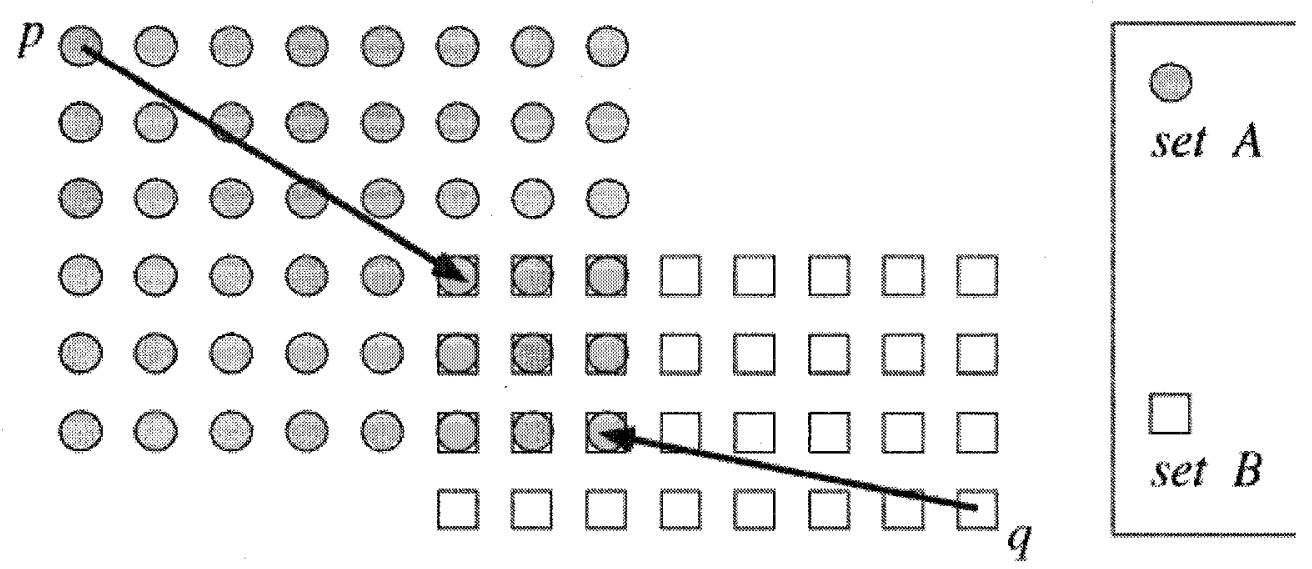

Figure 3.4 - Hausdorff's distance between sets A and B [15]

An algorithm for calculating the Hausdorff distance between any two sets $A$ and $B$ is presented in [15]. The distance can be calculated in $O(m n)$ time if a chamfer metric is used.

\subsubsection{Baddeley's Distance}

Equation 3.23 $B D=\left[\frac{1}{|U|} \sum_{p \in U}\left|\min _{q \in S U T} d(p, q)-\min _{q \in T S} d(p, q)\right|^{z}\right]^{\frac{1}{z}} \quad z \geq 1$

In this formula, $U$ denotes the pixel raster i.e. the set of all the pixels representing the image. This formula is effectively taking an average of the difference in the distances to the SUT and TS segmentation boundaries over all of the pixels in the image. As we are using an average, this measure is less noise-sensitive than Hausdorff's measure [16]. The parameter $z$ determines the relative importance of large localization errors i.e. a higher $z$ would penalize large localization errors more than smaller localization errors. Any distance between the edge pixel of the segmentation under test and the center of the true edge is a localization error. If $\mathrm{z}$ reaches infinity, Baddeley's distance will be close to Hausdorff's distance [20]. 


\subsubsection{Pratt's Figure of Merit}

Equation 3.24 PFOM $=\frac{1}{\max \{\mid b d(S U T))|,|(b d(T S)) \mid\}} \sum_{p \in b d(S U T)} \frac{1}{1+\frac{1}{9} \min _{q \in b d(T S)} d^{2}(p, q)}$

$b d(S U T)$ and $b d(T S)$ respectively represent the set of pixels in the boundaries of the segmentation under test and the true segmentation. Pratt's Figure of Merit (PFOM) [22] is always greater than zero and less than or equal to one. A higher value of PFOM leads to a higher similarity between the two segmentations. In the case where it is one, the segmentation under test is identical to the true segmentation. This measure is sensitive to over-segmentation and localization problems, but it does not take into account undersegmentation or shape errors. The insensitivity of PFOM to the pattern of pixel errors is seen in Figure 3.5 (taken from [23]). If A is the true segmentation, B and C will result in the same value for PFOM as they both have two corners of over-segmented pixels (the top and right corners). PFOM has not been theoretically proven, but is widely used as an empirical measure [16].
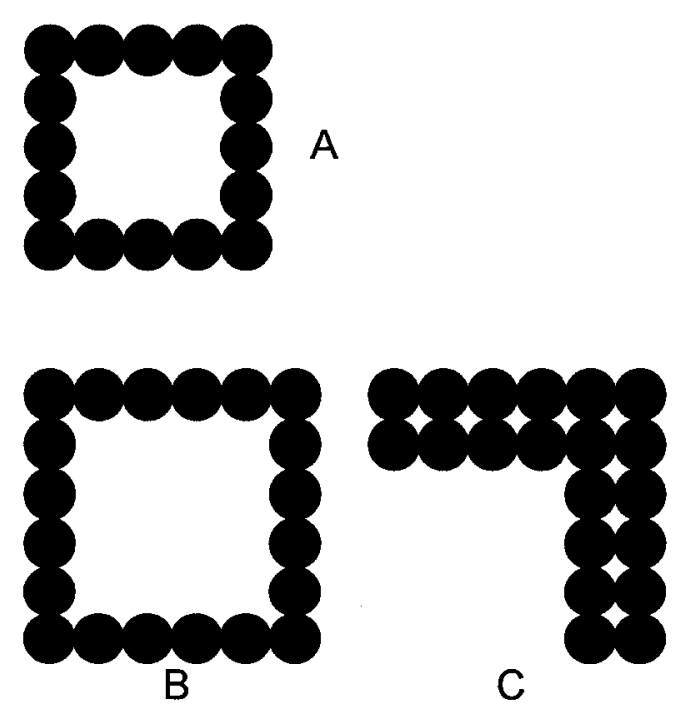

C

Figure 3.5 - PFOM rates $B$ and $C$ to be equally similar to $A$ [23] 


\subsubsection{Peli and Malah's Measures}

Peli and Malah have defined two measures which are simply the mean error (Equation 3.25) and mean squared error (Equation 3.26) of the distance between the boundary pixels of the segmentation under test and the boundary pixels of the true segmentation. These two measures are not normalized like Pratt's Figure of Merit and range between zero and infinity, larger values indicating more deviation between the segmentation under test and the true segmentation. If two segmentations are identical then the value of PMME and PMMSE will be zero.

Equation 3.25 $\quad P M M E=\frac{1}{|b d(S U T)|} \sum_{p \in b d(S U T)}\left(\min _{q \in b d(T S)} d(p, q)\right)$

Equation 3.26 PMMSE $=\frac{1}{|b d(S U T)|} \sum_{p \in b d(S U T)}\left(\min _{q \in b d(T S)} d(p, q)\right)^{2}$

\subsubsection{Odet's Measures}

Equation 3.27 $S O D I=\frac{1}{|b d(O S)|} \sum_{p \in b d(O S)}\left(\frac{\min _{q \in b d(T S)} d(p, q)}{d_{T H}}\right)^{n}$

Equation 3.28 $\quad S U D I=\frac{1}{|b d(U S)|} \sum_{p \in b d(U S)}\left(\frac{\min _{q \in b d(S U T)} d(p, q)}{d_{T H}}\right)^{n}$

In Equation 3.27 and Equation 3.28 above:

- OS is the set of over-segmented pixels in the segmentation under test (pixels that are labeled in the segmentation under test but not in the true segmentation)

- US is the set of under-segmented pixels (pixels that are labeled in the true segmentation but not in the segmentation under test).

- $d_{T H}$ is referred to as the saturation factor. $d_{T H}$ is application specific and determines what far (from a boundary) means. That is distances that are farther than $d_{T H}$ from the reference edge (edge in the true segmentation or the 
segmentation under test) are all considered to be the distance $d_{T H}$ from the reference edge. Dividing by $d_{T H}$ normalizes the measures.

- $\quad n$ is a scale factor that allows the assignment of different weights per pixel depending on the pixel's distance to the boundary of the true segmentation i.e. the pixels that are close to the boundary are weighted differently from the ones that have a distance to the true segmentation boundary close to $d_{T H}$ [16]. Setting the scale factor $n$ to a value more than 1 will result in considering the pixels close to the reference edge as correct and only putting emphasis on the pixels that have a distance close to the saturation factor $d_{T H}$ while setting it to a value that is more than zero and less than 1 will result in putting more emphasis on the pixels that are close to the reference edge. Setting $d_{T H}$ to smaller values leads to evaluation with a higher accuracy (as we are over-emphasizing minor discrepancies) but at the same time we may not be interested in minor discrepancies between the two segmentations [21].

SODI and SUDI are respectively used to detect the degree of over-segmentation and under-segmentation. The values of $n$ and $d_{T H}$ allow for the scaling of the measures. The non-scalable versions of SODI and SUDI are respectively referred to as ODI (Equation 3.29) and UDI (Equation 3.30).

Equation 3.29 $\quad O D I=\frac{1}{|b d(O S)|} \sum_{p \in b d(O S)} \min _{q \in b d(T S)} d(p, q)$

Equation 3.30 $\quad U D I=\frac{1}{|b d(U S)|} \sum_{p \in b d(U S)} \min _{q \in b d(S U T)} d(p, q)$

ODI and UDI simply take the average of the distance from the over-segmented boundary pixels to the true segmentation boundary (ODI) and the distance from the undersegmented boundary pixels to the segmentation under test boundary (UDI).

\subsubsection{Principal Axis Difference}

The principal axes of an object (in our case the labeled region of the segmentation) are in the direction of the eigenvectors of the covariance matrix representing that object. The 
covariance matrix (Equation 3.31) represents the covariance of the coordinates (Equation 3.32 ), in this case $3 \mathrm{D}$ Cartesian coordinates. The covariance matrix is a symmetrical matrix because $c_{i j}=c_{j i}\{i, j=1,2,3\}$.

Equation 3.31

$$
C=\left[\begin{array}{lll}
c_{11} & c_{12} & c_{13} \\
c_{21} & c_{22} & c_{23} \\
c_{31} & c_{32} & c_{33}
\end{array}\right]
$$

Equation 3.32 $\quad c_{i j}=\sum_{i, j=\{1,2,3\}} x_{i} x_{j}$

If we consider $\mathrm{C}$ as a transformation (a matrix that in the general case rotates and shifts the vectors that are applied to it), the eigenvectors are the vectors that do not rotate when the transformation is applied to them (Equation 3.33).

\section{Equation 3.33 $\quad C V_{i}=\lambda_{i} V_{i}$}

In Equation 3.33, $V_{i}(i>=1)$ are the eigenvectors of the covariance matrix and $\lambda_{i}(i>=1)$ are the associated eigenvalues of the eigenvectors. In the coordinate system represented by the eigenvectors (principal axes), the covariance matrix, shown by $C^{\prime}$ in this coordinate system will have the form of a diagonal matrix (Equation 3.34) (a matrix that only has nonzero elements on the diagonal) where the eigenvalues of the covariance matrix are on the diagonal of the matrix.

Equation 3.34

$$
C^{\prime}=\left[\begin{array}{ccc}
\lambda_{1} & 0 & 0 \\
0 & \lambda_{2} & 0 \\
0 & 0 & \lambda_{3}
\end{array}\right]
$$

As you see in the coordinate system represented by the principal axes, the covariances of the coordinates are zero with respect to each other, which means that the object is geometrically symmetrical around the principal axes (Figure 3.6). 


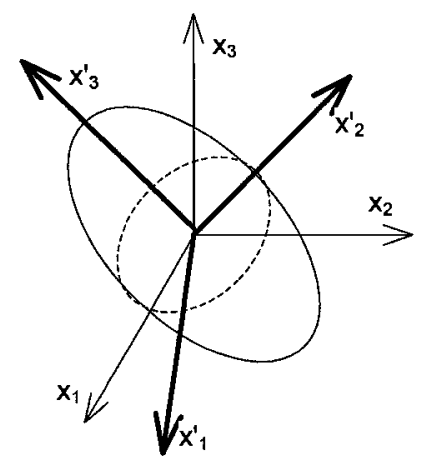

Figure 3.6 - Object is geometrically symmetrical around the principal axes $\left(x^{\prime}{ }_{1}, x^{\prime}{ }_{2}\right.$, $\left.\boldsymbol{x}_{3}^{\prime}\right)$

A closer look at $C^{\prime}$ shows that the eigenvalues are in fact the variances of the coordinates of the object points (segmentation pixels) in the principal coordinate system. We define the Principal Axis Difference comparison measure as follows:

Equation $3.35 \quad P A D=a b s\left(a b s\left(\max \left(\lambda_{i, T S}\right)\right)-a b s\left(\max \left(\lambda_{i, S U T}\right)\right)\right) \quad i=\{1,2,3\}$

PAD is calculating the difference of the largest variance of the pixels along the principal axes of the true segmentation and the segmentation under test. PAD solely concentrates on the shape of the segmentations and thus if the shape of the segmentation under test is the same as the true segmentation PAD would depict no difference between the segmentations, this is while the labeling in the segmentation under test may be located at a different location in the image from the expected location, making it an incorrect segmentation.

\subsubsection{RMS surface distance}

The RMS surface distance is defined as follows [17]:

Equation 3.36 $R M S S D=\sqrt{\frac{\sum_{p \in b d(S U T)}\left[\min _{q \in b d(T S)} d(p, q)\right]^{2}+\sum_{q \in b d(T S)}\left[\min _{p \in b d(S U T)} d(q, p)\right]^{2}}{|b d(S U T)|+|b d(T S)|}}$ 
RMSSD (Root Mean Square Surface Distance) is effectively taking a root mean square type average of the distances between the boundary (surface) pixels of the segmentation under test and the true segmentation over all the boundary pixels of both segmentations.

\subsubsection{Volume difference measures}

The following measures simply calculate the volume difference of the true segmentation and the segmentation under test in 3D scenarios.

- Absolute value of volume difference:

Equation 3.37 $A V D=\left|V o l_{s u t}-V o l_{t s}\right|$

- Absolute value of normalized volume difference:

Equation 3.38 $A N V D=\frac{\left|\operatorname{Vol}_{s u t}-\operatorname{Vol}_{t s}\right|}{V o l_{t s}}$

\subsection{Machine learning techniques}

Machine learning techniques are algorithms and methods that are used in order for a computer system to learn. Machine learning has applications in different areas such as natural language processing, stock market analysis, search engines, medical diagnosis, object recognition in computer vision and speech and handwriting recognition. One main application of machine learning is data mining. In data mining, the computer system learns special patterns of interest from large data sets.

The input data to a machine learning algorithm consists of a set of instances that are each characterized by the values of a number of attributes. The output of the machine learning algorithm is some form of representation of the learnt knowledge from applying the machine learning algorithm to the input [24].

Machine learning algorithms can be categorized into three types: 
1) Classification algorithms: These algorithms associate a class to an unknown instance (i.e. an instance which the associated class is not known.). These are the type of algorithms that we are interested in, in this thesis.

2) Association algorithms: These algorithms find associations between the attributes that characterize the instances.

3) Clustering: These algorithms divide the instances into natural groups.

We will only concentrate on classification algorithms in this thesis. A classification algorithm is trained via instances that have known classifications (training instances) to create a model that can predict the class of unknown instances. This learnt model is referred to as a classifier.

Some important categories of classification algorithms are as follows:

1) Rule-induction techniques: In these methods the classifier is represented by a set of rules that each associate a particular class to a certain relationship among the attributes.

2) Decision Trees: A divide-and-conquer approach is taken to construct a decision tree for classifying instances.

3) Probabilistic models: These models output the probability that each instance can belong to each class. The instance will be classified to the class with the highest probability.

4) Linear Models: A weighted linear function is learnt by solving an optimization problem. This linear function is used to classify unknown instances.

5) Instance-based learning: In this technique, the training instances are stored verbatim and methods such as finding the nearest neighbor are used to find the nearest training instance to the unknown instance, in which case the unknown instance is predicated to have the same class as the nearest training instance [24]. 
Decision trees and rule induction techniques are of particular interest as their models are readily interpretable by experts using them. The learnt model (classifier), output from the machine learning algorithm, has to be validated. In machine learning systems, the input data is divided into two main sets: the training set and the test set. The training set is used to construct the model and the test set is used to validate the model. Validating the model by deriving the error rate using the training model itself would lead to very optimistic results. Thus the test set should preferably be independent of the training set. Both the training and test sets should have representative instances of all types in the underlying problem so that the model is thorough and the validation of the model is also comprehensive. One of the techniques used to guarantee that all the representative samples are taken into account in the training or test sets is referred to as stratification. Stratification attempts to randomly choose the instances such that all the classes (that each of the instances belongs to) are represented in the right proportion, i.e., no class is over-represented by too many samples or under-represented by scarce samples. In an effort towards finding a reasonable error estimate to validate the model, a method referred to as cross-validation is commonly used. In this method, the available data is divided into a number of folds, each time one fold is used as the test set and the remainder is used for the training data. The cross-validation error estimate is the average of the error estimates that are calculated using each fold. Each error estimate could be simply the division of the number of misclassified test instances by the total number of test instances. Usually stratification is also used in cross-validation. For example, a 10fold cross-validation along with stratification is referred to as stratified 10-fold crossvalidation which is usually the standard way of measuring the error rate of a learning method. In order to reduce any bias towards choosing the training and test set instances it is common to repeat stratified 10-fold cross-validation 10 times and get the average of the error estimates obtained from each cross-validation run. Other error estimates such as leave-one-out cross-validation are also used. In this approach each time one instance is left out for testing while the rest of the instances are used for training. This approach is repeated until all of the instances are used once for testing the learning method. Leaveone-out cross-validation does not involve any stratification as it uses the whole data set for both training and testing purposes, and is therefore computationally expensive. 
A two-class prediction involves four outcomes as shown in Table 3.1. Different performance metrics can be defined using the terms defined in Table 3.1.

\begin{tabular}{|c|c|c|c|}
\hline \multicolumn{2}{|c|}{ Two-class prediction } & \multicolumn{2}{c|}{ Predicted Class } \\
\cline { 3 - 4 } \multicolumn{2}{|c|}{} & Yes & No \\
\hline \multirow{2}{*}{ Actual Class } & Yes & True Positive (TP) & False Negative (FN) \\
\cline { 2 - 4 } & No & False Positive (FP) & True Negative (TN) \\
\hline
\end{tabular}

Table 3.1 - Two-class prediction outcomes

We are going to use three performance metrics to compare the performance of different classifiers in this thesis: Accuracy, Kappa Statistic and the area under the ROC curve.

Accuracy is the success rate of the classifier which is in effect the complement of the error rate:

Equation 3.39 Accuracy $=\frac{T P+T N}{T P+T N+F P+F N}$

In order to take out the chance factor from the classifier predictions, the Kappa Statistic is defined as follows:

Equation 3.40

$$
\text { KappaStatistic }=\frac{T P_{C}+T N_{C}-\left(T P_{R}+T N_{R}\right)}{\text { Number of test instances }-\left(T P_{R}+T N_{R}\right)}
$$

In Equation 3.40, The $C$ subscript refers to the classifier and the $R$ subscript refers to a random predictor. In effect, the Kappa Statistic shows how much better we can perform in comparison with a random predictor by finding out the ratio of the extra correct predictions that the classifier makes out of the number of instances that have been incorrectly classified by the random predictor. The maximum value the Kappa Statistic can attain is 1 which indicates that the classifier has had no predictions made by chance.

Graphical techniques such as ROC curves are also a standard method to compare the performance of different classifiers. ROC stands for Receiver Operating Characteristic, which is a term that is used in signal detection to depict the hit rate versus the false alarm 
rate. In other words, it provides insights into the cost-effectiveness of the classifier. In machine learning terms, ROC curves plot the true positive rate (tp) versus the false positive rate (fp), which are defined in Equation 3.41 and Equation 3.42.

Equation 3.41 $\quad t p=\frac{T P}{T P+F N} * 100 \%$

Equation $3.42 \quad f p=\frac{F P}{F P+T N} * 100 \%$

The area under the ROC curve is a single number that can be used similarly to the Accuracy and Kappa Statistic as a performance indicator. The larger the area, the better the classifier performs. This makes sense intuitively as a larger area would mean that the ROC curve has had a higher tp to fp ratio at most times. 


\section{IMAGE SEGMENTATION EVALUATION ORACLE DESIGN}

Figure 4.1 depicts a semi-automatic solution that is proposed in this thesis for image segmentation evaluation.

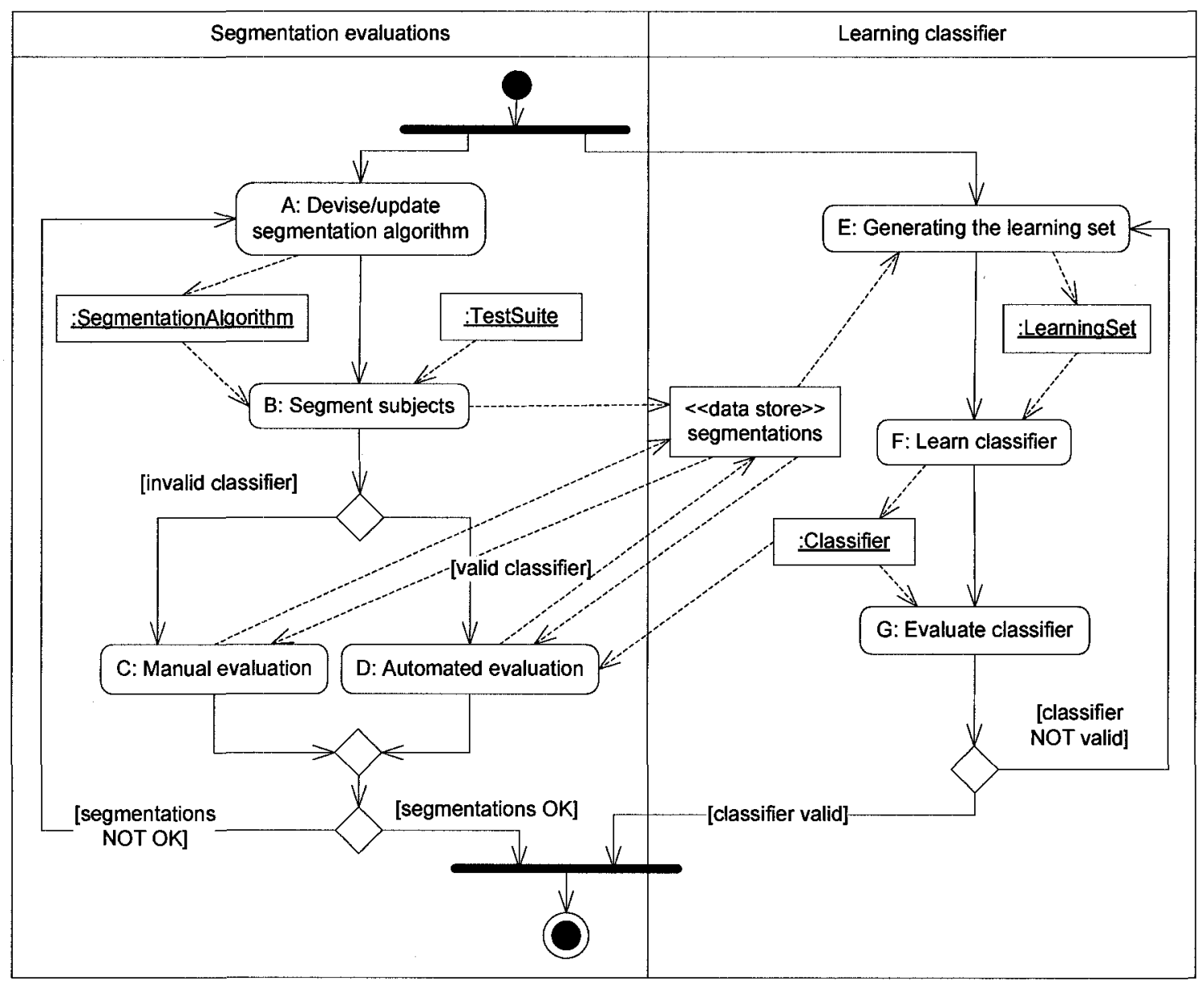

Figure 4.1 - Semi-automated image segmentation algorithm evaluation process

Two series of activities take place concurrently in this process, as illustrated by the two swimlanes, named Segmentation evaluations and Learning classifier. The former swimlane entails activities $A$ to $D$, and the latter entails activities $E$ to $G$. Some of the activities in each series depend on the output of activities in the other series which is 
further explained below (e.g., activity E takes an input produced by activity B, activity D takes an input produced by activity F). In the left swimlane (the Segmentation evaluations swimlane), the image segmentation algorithm is revised (activity A) and the segmentations produced by each revision of the image segmentation algorithm (activity B) are evaluated for their correctness. These are evaluated either manually by experts (activity C) or automatically - see below (activity D). In the right swimlane (the Learning classifier swimlane), a classifier is learnt. Once a valid classifier is learnt, the segmentation evaluation in the evaluation thread is done automatically (activity D). These two series of activities are further described below in Sections 4.1 and 4.2, respectively. We then discuss the architecture of our tool support in Section 4.3.

\subsection{Segmentation evaluations swimlane}

This swimlane has four activities: activities A to D. The first time the Devise/Update segmentation algorithm activity (Activity A) is performed, an initial version of the image segmentation algorithm is devised. Each time this activity is repeated, i.e., when the overall evaluation of the segmentations produced by the image segmentation algorithm fails, the image segmentation algorithm is revised. Further revisions of the image segmentation algorithm are made in subsequent iterations until a satisfactory set of segmentations are produced by a revision of the image segmentation algorithm.

During the Segment subjects activity (Activity B), the segmentation algorithm produced in activity A is used to segment a set of sample subject images or test cases (input named Test suite). This results in a set of segmentations, each segmentation corresponding to one sample subject (test case). We refer to the segmentations obtained from revision i of the segmentation algorithm (i.e., during the $\mathrm{i}^{\text {th }}$ iteration of this swimlane) as segmentation set set $_{i}$.

The segmented images are evaluated either manually or automatically in the next two activities ( $C$ and D), depending on whether a valid classifier has been learnt. If a valid classifier has not yet been learnt (Section 4.2), the segmentations have to be manually evaluated (Activity C-Manual evaluation of segmentations). Each segmentation is graded by both the image segmentation algorithm designer and medical experts and is 
considered as correct if it receives a reasonable grade (The grading scale is to be decided by the algorithm designer and the medical expert.). If a valid classifier is available (Section 4.2), the evaluation is done automatically: activity Automated evaluation of segmentations (Activity D). In this case, the classifier predicts the grade of each segmentation without the intervention of a human expert (algorithm designer or medical expert). During automated evaluation, segmentations produced by the current revision (i) of the segmentation algorithm are compared by the classifier (using similarity measures) to segmentations produced by the previous revisions $(j<i)$ of the segmentation algorithm. The details of how this is done are explained in Section 4.3.

Table 4.1 shows how the correctness of segmentation $\mathrm{n}$ produced by revision $\mathrm{i}$ of the segmentation algorithm $\left(\mathrm{Sn}_{\mathrm{n}} \mathrm{i}\right)$ can be predicted with the learnt classifier. If the classifier classifies segmentation $\mathrm{S}_{n, i}$ to be consistent (with respect to a number of similarity measures) with a correct segmentation then segmentation $\mathrm{Sn}_{\mathrm{n}} \mathrm{i}$ is predicted to be correct. If segmentation $\mathrm{Sn}$,i is classified to be inconsistent with a correct segmentation or consistent with an incorrect segmentation then it is predicted to be incorrect. In the case where segmentation $i$ is inconsistent with an incorrect segmentation, it cannot be considered as either correct or incorrect and the correctness of segmentation $i$ has to be manually evaluated by the expert.

\begin{tabular}{|l|l|l|}
\hline Evaluation of $\mathbf{S n}, \mathbf{j}(\mathbf{j}<\mathbf{i})$ & $\begin{array}{l}\text { Predicted consistency of } \\
\text { segmentation pair }(\mathbf{S n}, \mathbf{j}-\mathbf{S n}, \mathbf{i})\end{array}$ & Evaluation of $\mathbf{S n}, \mathbf{i}$ \\
\hline Correct & Consistent & Correct \\
\hline Correct & In-Consistent & In-correct \\
\hline In-correct & Consistent & In-correct \\
\hline In-correct & In-consistent & Requires manual validation \\
\hline
\end{tabular}

Table 4.1 - Mapping between classifier results and the evaluation of the unknown image segmentation

In both activities $\mathrm{C}$ and $\mathrm{D}$, if the image segmentations do not receive an overall reasonable grade, we go back to activity $\mathrm{A}$ where the image segmentation algorithm is 
revised. Otherwise, the testing process ends and the current revision of the image segmentation algorithm is deemed to be correct.

The idea of using multiple versions of the segmentation algorithm to find out whether the output of the segmentation algorithm is correct is similar to multi-version programming mentioned in Chapter 2. Note that in our case the multiple versions are a natural result of the evolution of the software until an acceptable version is devised, so the cost involved in producing extra versions of the same software that are solely for testing purposes is not applicable in our methodology.

\subsection{Learning classifier swimlane}

This swimlane has three activities: activities E to G. During Activity E (Generating the learning set), pairs of segmentations obtained from multiple revisions of the image segmentation algorithm (current revision $\mathrm{i}$, and revision $\mathrm{j}, \mathrm{j}<\mathrm{i}$ ) are compared using a set of similarity measures (Section 3.1). At least the first two sets of segmentations generated by the first two revisions of the segmentation algorithm are required to get the first set of similarity measurements (i.e., the first set of evaluated segmentation pairs from versions 1 and 2 of the segmentation algorithm). In other words, at least two iterations of the Segmentation evaluations swimlane (with manual evaluation in Activity C) are necessary.

Pairing segmentations of the same subjects across two segmentation sets set $_{i}$ and set $_{j}$ results in three distinct subsets of paired segmentations. The first set is composed of the pairs of segmented subjects that were both deemed correct by an expert, denoted by set (i.e., " $y$ ' for "yes" for the two versions). The second set is composed of the pairs of segmented subjects where either the first or second segmented subject was deemed incorrect, denoted by set $t_{y n}$ (one is correct, i.e., one ' $y$ ', and one is incorrect, i.e., one ' $n$ '). The third set is the set of all the pairs of segmented subjects that were both considered incorrect, denoted by set $_{n n}$.

The learning algorithm (Activity F) does not use $s t_{n n}$ as the information obtained from comparing two incorrectly segmented subjects would not help the learning algorithm 
construct a classifier to recognize diagnostically equivalent segmentations. Two segmentations may be incorrect for two completely different reasons and thus we cannot categorize them as consistent with each other. Table 4.2 explains how we categorize a pair of segmentations to be consistentlinconsistent where $S n, i$ and $S n, j$ are two segmentations obtained from subject $\mathrm{n}$ using versions $\mathrm{i}$ and $\mathrm{j}$ of the segmentation algorithm, respectively.

Similarity measures (Section 3.1) are used to compare the paired segmented subjects in sets $s^{2} t_{y y}$ and set $_{y n}$. In other words, for each pair of segmentation algorithms applied to the same set of subjects, Activity E generates a set of tuples with a size equal to the number of subjects considered: $\left(\mathrm{sm}_{\mathrm{i} 1}, \ldots, \mathrm{sm}_{\mathrm{ik}}\right.$, Consistency), where $s m_{i j}$ denotes similarity measure $j$ on subject pair $i$ and "Consistency" can take two values: $y y$ or $y n$. $k$ is the number of similarity measures.

\begin{tabular}{|l|l|l|}
\hline Manual evaluation of $\mathbf{S n}, \mathbf{i}$ & Manual evaluation of $\mathbf{S n}, \mathbf{j}$ & Class \\
\hline Correct & Correct & Consistent \\
\hline Correct & Incorrect & Inconsistent \\
\hline Incorrect & Correct & Inconsistent \\
\hline Incorrect & Incorrect & Unusable (not a class) \\
\hline
\end{tabular}

Table 4.2 - Consistency of two segmentations

This set of tuples is the training set of instances that is fed into the machine learning algorithm: activity $\mathrm{F}$ (Learn classifier). The machine learning algorithm learns which ranges of these measures, and which of these measures, correspond to a diagnostically consistent pair of segmentations, and which ranges (and measures) correspond to a diagnostically inconsistent pair of segmentations. A diagnostically consistent pair of segmentations refers to two segmentations that both lead to the same diagnostic by the expert (algorithm designer or medical expert).

The learnt classifier (activity $F$ ) is validated using techniques such as 10 -fold crossvalidation (Section 3.2) in activity G (Evaluate classifier). A classifier is deemed valid if the average error estimate is considered to be sufficiently low to be used in practice. This 
means that the classifier correctly predicted a reasonable proportion of segmentations under test to be consistent or inconsistent with a reference segmentation of the same subject, or in other words predicted if the segmentation under test is correct or not. Once the classifier is learnt, the evaluation in the Segmentation evaluations swimlane can be done automatically (activity D). In the case where we do not succeed in learning a classifier with very high accuracy, there are always some branches (in the case of decision trees) or rules (in the case of rule induction techniques) where the classifier has a very low error rate and its predictions can be trusted with high confidence. If the classifier can provide accurate predictions for a significant number of cases, then the classifier is a good candidate for automatic evaluation.

\subsection{Tool support architecture}

The structural components in the design of the automated oracle are shown in Figure 4.2. As shown in Figure 4.2, the oracle design is generic and does not have to be restricted to the image segmentation evaluation domain. The outputs (in our case the segmentations) from version $\mathrm{V}_{\mathrm{i}}(1 \leq i \leq n$ where $n$ is the total number of versions of the Software Under Test (SUT) until its depicted as valid) of the software under test (in our case the software under test is the segmentation algorithm) are fed into the oracle and the oracle determines the correctness of the outputs. 


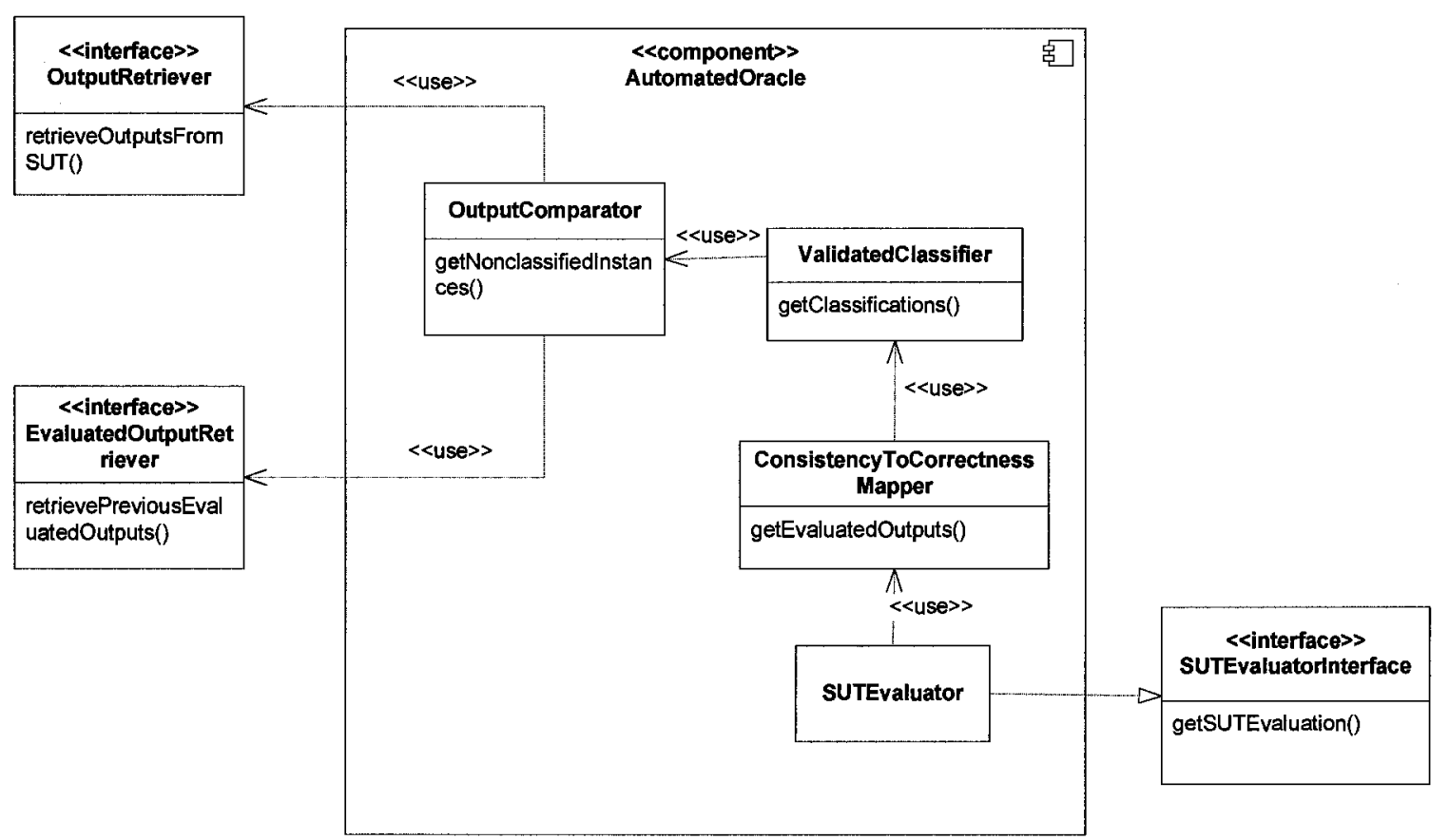

Figure 4.2 - Structural components of the automated oracle

To do so, the OutputComparator class is used to retrieve the outputs from the current version of the SUT via the OutputRetriever interface. Each output $\mathrm{O}_{\mathrm{ji}}$ $(1 \leq j \leq$ total_num_subjects, $1 \leq i \leq$ total_num_software_versions $)$ produced from input $\mathrm{I}_{\mathrm{j}}$ (the input in our case is the $3 \mathrm{D}$ volume CT-scan of the subject) is first compared to the outputs that have been produced from the same input $\mathrm{I}_{j}$ using previous versions of the software under test (version $<\mathrm{V}_{\mathrm{i}}$ ). The previously evaluated outputs have been retrieved via the EvaluatedOutputRetriever interface which may be provided by an external database used for storing the previously evaluated outputs. The output that is being tested will be referred to as the Output Under Test (OUT). Table 4.3 shows the contents stored in the external database more vividly. 


\begin{tabular}{|l|l|l|l|l|}
\hline Input & SUT V & SUT V & $\ldots$ & SUT V V \\
\hline $\mathrm{I}_{1}$ & $\mathrm{O}_{11} \backslash$ Correct & $\mathrm{O}_{12} \backslash$ Correct & & $\mathrm{O}_{1(\mathrm{i}-1)} \backslash$ Correct \\
\hline $\mathrm{I}_{2}$ & $\mathrm{O}_{21} \backslash$ Incorrect & $\mathrm{O}_{22} \backslash$ Incorrect & & $\mathrm{O}_{2(\mathrm{i}-1)} \backslash$ Incorrect \\
\hline $\mathrm{I}_{3}$ & $\mathrm{O}_{31} \backslash$ Correct & $\mathrm{O}_{32} \backslash$ Incorrect & & $\mathrm{O}_{3(\mathrm{i}-1)} \backslash$ Correct \\
\hline $\mathrm{I}_{4}$ & $\mathrm{O}_{41} \backslash$ Incorrect & $\mathrm{O}_{42} \backslash$ Incorrect & & $\mathrm{O}_{4(\mathrm{i}-1)} \backslash$ Correct \\
\hline$\ldots$ & $\ldots$ & $\ldots$ & $\ldots$ & $\ldots$ \\
\hline
\end{tabular}

Table 4.3 - Table of all outputs produced by the previous versions of the SUT (version < Vi) and their corresponding evaluations

The OutputComparator uses the comparison measures defined in Sections 3.1.1, 3.1.2 and 3.1.3 to compare $\mathrm{O}_{\mathrm{ji}}(\mathrm{OUT})$ with the outputs: $\mathrm{O}_{\mathrm{j}(\mathrm{i}-1)}, \mathrm{O}_{\mathrm{j}(\mathrm{i}-2)}, \ldots, \mathrm{O}_{\mathrm{j} 2}, \mathrm{O}_{\mathrm{j} 1}$ and for each comparison (for example if the current version of the SUT is $\mathrm{V}_{10}$, one comparison could be between $\mathrm{O}_{2,10}$ (OUT) and the output produced by the $8^{\text {th }}$ version of the SUT i.e. $\mathrm{O}_{2,8}$; the input associated with both outputs is $I_{2}$ ) will produce a tuple of the comparison measure calculations $\left(\mathrm{m}_{1}, \mathrm{~m}_{2}, \ldots, \mathrm{m}_{\mathrm{z}}\right)$ where $z$ is the number of defined comparison measures. The ValidatedClassifier (a classifier that has been validated by Activity $\mathrm{G}$ ) retrieves these tuples which serve as the non-classified instances and classifies them producing tuples in the form $\left(\mathrm{m}_{1}, \mathrm{~m}_{2}, \ldots, \mathrm{m}_{\mathrm{z}}\right.$, consistentlin-consistent) which now contains the classification. The Consistency to Correctness Mapper class uses the information provided in Table 4.3 and the logic explained in Table 4.1 to map the consistency classifications to correctness classifications. Table 4.4 shows an example on how this is done. 


\begin{tabular}{|l|l|l|l|}
\hline $\begin{array}{l}\text { Output that OUT } \\
\text { has been compared } \\
\text { to }\end{array}$ & $\begin{array}{l}\text { Correctness } \\
\text { Classification of Previous } \\
\text { Outputs }\end{array}$ & $\begin{array}{l}\text { Consistency } \\
\text { Classification }\end{array}$ & $\begin{array}{l}\text { Correctness } \\
\text { Classification of } \\
\text { OUT }\end{array}$ \\
\hline $\mathrm{O}_{9.6}$ & Correct & Consistent & Correct \\
\hline $\mathrm{O}_{9.5}$ & Incorrect & Inconsistent & Unknown \\
\hline $\mathrm{O}_{9,4}$ & Correct & Inconsistent & Incorrect \\
\hline $\mathrm{O}_{9.3}$ & Correct & Consistent & Correct \\
\hline $\mathrm{O}_{9,2}$ & Incorrect & Consistent & Incorrect \\
\hline $\mathrm{O}_{9,1}$ & Correct & Consistent & Correct \\
\hline
\end{tabular}

Table 4.4 - An example showing how the Consistency to Correctness Mapper module obtains the correctness of the OUT

In this example, OUT is the output produced from the $7^{\text {th }}$ version $\left(\mathrm{V}_{7}\right)$ of the segmentation algorithm from input $9\left(\mathrm{I}_{9}\right)$ i.e. output $\mathrm{O}_{9,7}$. The Consistency to Correctness Mapper uses a policy to make the final decision as to whether OUT is correct or not. This policy could be taking a majority vote (in this example, OUT has been determined to be correct in 3 instances, while it has been deemed incorrect in 2 instances, so OUT is evaluated to be correct) or just taking the evaluation made using the comparison with the last version of the SUT. The assumption here is that the last version of the SUT is the superior version of all the previous versions. We cannot automatically evaluate the correctness if all the outputs from the previous versions of the SUT are evaluated to be incorrect and the classifier has classified OUT to be inconsistent with all of them (the unknown case in Table 4.1) or in the case where there is an equal number of corrects and in-corrects (this is only an issue when using the majority vote policy). The evaluated outputs (outputs classified as correctlin-correct by the ValidatedClassifier) are retrieved by the SUTEvaluator class that uses some defined criteria to determine whether we have enough correct outputs produced by the current version of the software under test to evaluate this version as a valid version and thus the final version of the software under test. The SUTEvaluator class has to provide an interface for the test monitor (the module that runs the test cases) to retrieve the oracle's evaluation on the current SUT. This is provided through the SUTEvaluatorInterface. 


\section{CASE STUDY: LEFT HEART VENTRICLE SEGMENTATION EVALUATION}

The case study that is used in this research work is evaluating the correctness of the segmentation algorithms that are devised to segment the left heart ventricle. Once the left ventricle is identified, its volume is computed for diagnostic purposes; this is however not in the scope of this thesis. Before we start talking about the details of the case study and how we have used the solution proposed in Chapter 4 to solve the problem of evaluating medical image segmentations in a timely fashion we explain why the software under test (the medical image segmentation algorithms) can be considered as un-testable programs with a discussion based on the three criteria given for un-testable programs in [1] (refer to Chapter 2).

In order to verify the correctness of a devised medical image segmentation algorithm, the segmentation algorithm is applied to a sample subset of subjects; if a predefined percentage of the output segmentations are deemed to be correct, the segmentation algorithm is deemed correct. A medical expert can manually segment a subject, so the output of the segmentation algorithm is known, but it is very dependent on the availability of the medical expert and is very time-consuming considering the large size of the sample test-set. Typically, hundreds of subjects are used. As the correct segmentation is usually not available we can consider the output of the segmentation algorithm to be "unknown" (category 1 of untestable software described in Chapter 2).

The process of devising the final segmentation algorithm is an iterative process requiring many revisions of the initial segmentation algorithm. As the same medical experts are not always available during the evaluation of the image segmentation algorithm, different versions of the image segmentation algorithm may be evaluated by different medical experts. This leads to subjectivity in the overall assessment of the quality of each version of the image segmentation algorithm as different experts may impose different weights to different aspects of their assessment. This implicitly indicates the segmentation algorithm 
is being evaluated with respect to (slightly) different specifications (the oracle problem is in category 3 of untestable software described in Chapter 2).

Considering the problem of testing the correctness of medical image segmentation algorithms lies in categories 1 and 3, in the context explained in [1] we could refer to the medical segmentation algorithm as an untestable program. In this case study we tackle the problem of testing medical image segmentation algorithms using the approach defined in chapter 4 . In this approach we only need medical expert intervention for the first few revisions of the segmentation algorithm (two revisions proves to be enough in this case study), which partly relieves the problem of having a large dependency on the medical experts to evaluate each and every revision of the segmentation algorithm because of lack of knowledge of what the correct segmentation for each subject is (category 1). If we get the majority vote of the medical experts in the evaluation of the first few revisions of the segmentation algorithm, we do not encounter subjectivity in the evaluation of the different revisions of the segmentation algorithm as the evaluation is done automatically based on the majority vote done for the first few revisions and does not depend on a possibly different medical expert's evaluation for different revisions of the segmentation algorithm (category 3).

This chapter is divided into 9 sections. In order to give the reader a better idea about the left heart ventricle segmentation, some segmentations are shown in Section 5.1. Section 5.2 details the setup of the experiments. In order to give some insight into how the classification algorithms used in this thesis work, the three main data mining techniques used in this case study (J48, PART and JRIP) are described in Section 5.3. Section 5.4 describes how the defined attributes (comparison measures) are correlated using Spearman coefficients. The different attribute sets used for training the classifiers and the reasoning behind choosing them is explained in Section 5.5. Section 5.6 shows the classifiers output from the data mining techniques and explains how to interpret them. The performance of the classifiers is compared in Section 5.7 using metrics such as Accuracy, Kappa Statistic and the area under the ROC curve. The analysis and implementation tools used in this case study are described in Section 5.8 explaining their 
pros and cons. A discussion of the calculation time of the different types of comparison measures is given in Section 5.9 concluding the chapter in Section 5.10.

\subsection{Examples}

We show here some figures showing the left heart ventricle segmentations. Each figure is a slice of the CT-Scan of a different patient's heart where the left ventricle is segmented by labeling the representative pixels with green using some version of the segmentation algorithm. Figure 5.1 shows a correct segmentation while Figures Figure 5.2, Figure 5.3 and Figure 5.4 show incorrect segmentations. Figure 5.2 shows a typical oversegmentation scenario (the most common problem in this case study) where the left atrium is also labeled. In Figure 5.3 the segmentation algorithm has missed the left ventricle and labeled the right ventricle instead. Severe undersegmentation is seen in Figure 5.4 where almost nothing is labeled in this slice of the heart. These are some examples of incorrect segmentations in this case study. More detailed discussion on the manual evaluation of each segmentation is out of the context of this research work and requires background in the heart anatomy. Also note that these figures only outline the segmented region in one slice of the heart. In order to evaluate the correctness of a segmentation, all the CT-Scan slices of the heart have to be looked at by an expert where an overall assessment is then given as to whether the segmentation is correct or not. Minor discrepancies in a few slices may be ignored by the expert. As explained earlier the tolerance level for accepting a segmentation as correct may be different for each expert. 


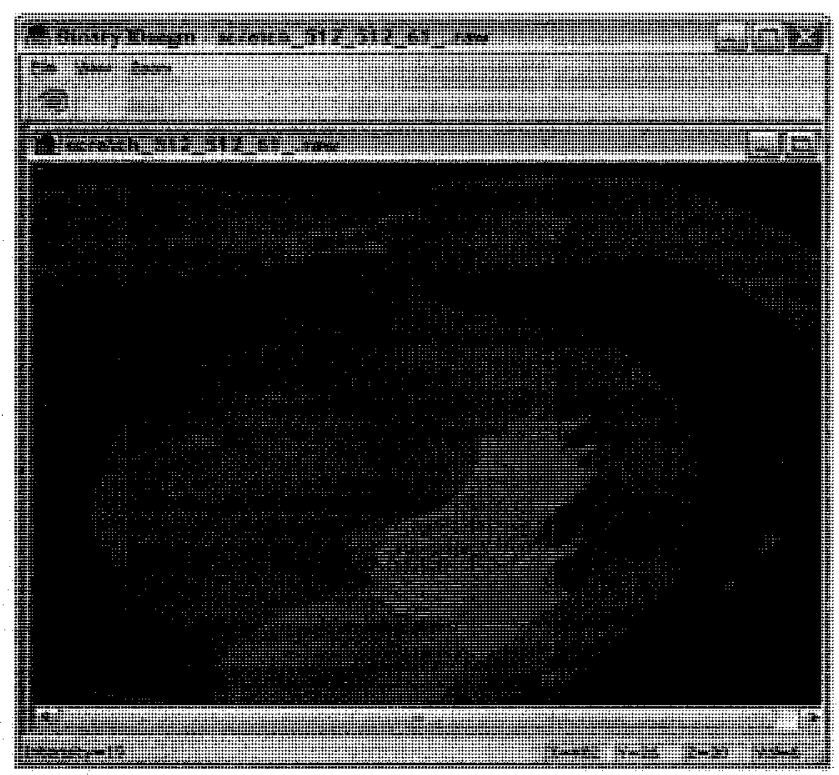

Figure 5.1 - Correct Left Heart Ventricle Segmentation

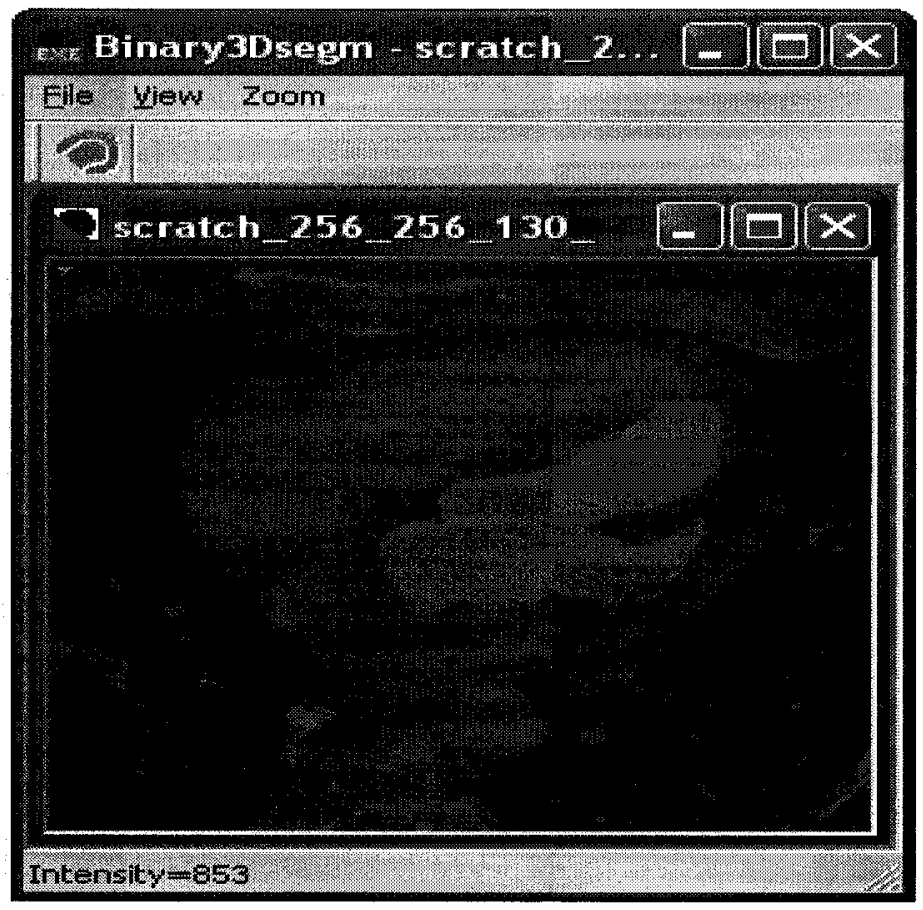

Figure 5.2 - Incorrect Segmentation (oversegmentation to the left atrium) 


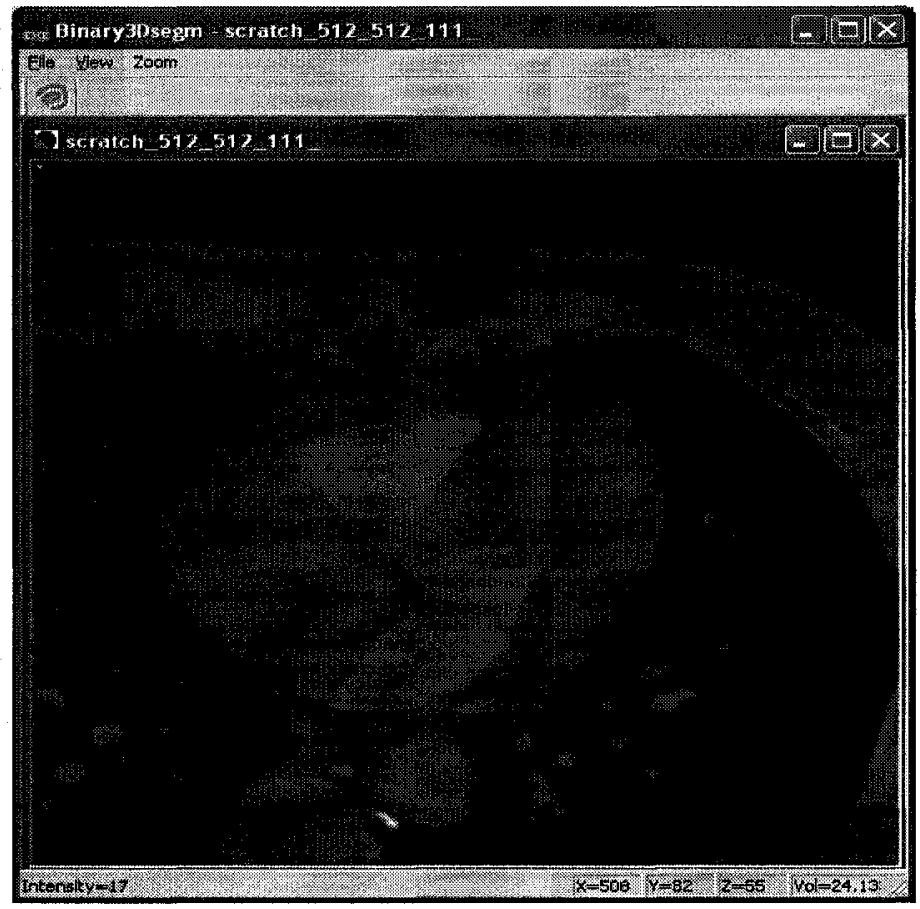

Figure 5.3 - Incorrect Segmentation (Segmenting an incorrect region i.e. the right ventricle)

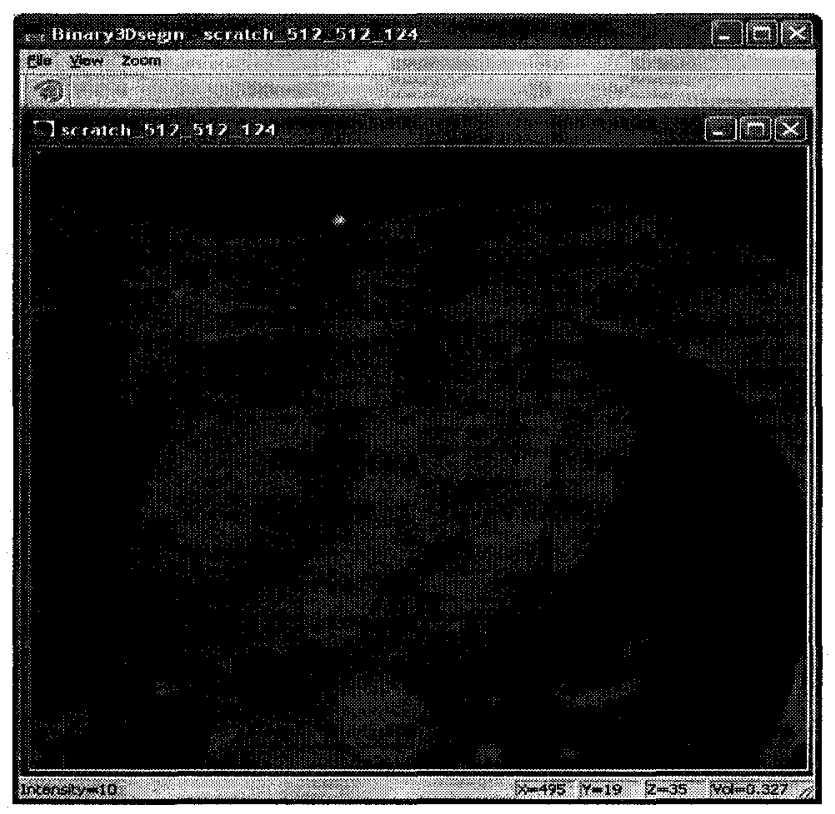

Figure 5.4 - Incorrect Segmentation (severe undersegmentation) 


\subsection{Experiment setup}

Table 5.1 summarizes the details of the case study. CT-Scan images of the heart of 50 patients have been taken at different phases of the cardiac cycle. Each phase is a point of time in the cardiac cycle. At each phase, the 3D volume of the heart is represented by a set of 2D CT-Scan images obtained from the heart in that phase (in this case study, this set could have from 53 to 460 images). Each of the 2D CT-Scan images depicts either a 256 by 256 or 512 by 512 pixel slice of the heart represented in the DICOM (Digital Imaging and Communication in Medicine) format, which is a standard file format for representing medical images. The obtained 3D volume of the heart in each phase constitutes a test case or subject (181 in this case study).

\begin{tabular}{|l|l|}
\hline Segmentation Target & Left ventricle of the heart \\
\hline Number of patients & 50 \\
\hline Number of test cases in test suite & 181 \\
\hline Number of attributes (measures) & 18 \\
\hline Number of positive instances & 104 \\
\hline Comparison measure types & Overlap, Volume Difference, Geometrical \\
\hline Machine learning algorithms & C4.5, JRIP, PART \\
\hline Classifier evaluation technique & Stratified 10-fold cross-validation \\
\hline Performance Metrics & Accuracy, Kappa Statistic, Area under ROC curve \\
\hline
\end{tabular}

Table 5.1 - Details of case study

Two revisions of the image segmentation algorithm have been used to obtain two sets of image segmentations of the subjects. The image segmentations have been manually evaluated by the author of this writing with the help of Siemens Corporate Research experts (Activity $\mathrm{C}$ in Chapter 4 ). The grading scheme is a binary grading scheme; an image segmentation is either evaluated as correct or incorrect. In order to generate the training set (Activity E) for the machine learning algorithms, for each subject, the segmentations produced by image segmentation algorithm versions 1 and 2 are compared using different combinations of the comparison measures in Table 5.2 (please refer to 
Section 3.1 for a description of these measures). The 18 measures in Table 5.2 are the attributes of the instances that are fed into the machine learning algorithms. In this case study we have 181 training instances corresponding to the 181 test cases (patient-phase CT-Scans). Each instance $i$ is represented by the tuple $\left(\mathrm{dsc}_{\mathrm{i}}, \operatorname{tc}_{\mathrm{i}}, \operatorname{tpvf}_{\mathrm{i}}, \operatorname{fpvf}_{\mathrm{i}}, \operatorname{avd}_{\mathrm{i}}, \operatorname{anvd}_{\mathrm{i}}\right.$, adbd $_{i}, h_{i}$, bd $_{i}$, pmme $_{i}$, pmmse $_{i}$, pfom $_{i}$, sodi $_{i}$, sudi $_{i}$, odi $_{i}$, udi $_{i}$, pad $_{i}$, rmssd $_{i}$, consistency) when all attributes are used to train the classifier. The consistency classification has been obtained from Activity $\mathrm{C}$. These instances are fed into each machine learning algorithm to learn a classifier.

\begin{tabular}{|l|l|l|}
\hline Measure & Type & Description \\
\hline DSC & Overlap & Dice Similarity Coefficient \\
\hline TC & Overlap & Tanimoto Coefficient \\
\hline TPVF & Overlap & True Positive Volume Fraction \\
\hline FPVF & Overlap & False Positive Volume Fraction \\
\hline AVD & Volume Difference & Absolute value of Volume Difference \\
\hline ANVD & Volume Difference & Absolute value of Normalized Volume Difference \\
\hline ADBD & Geometrical & Average Distance to Boundary Difference \\
\hline HD & Geometrical & Hausdorff Difference \\
\hline BD & Geometrical & Baddeley Difference \\
\hline PMME & Geometrical & Peli Malah Mean Error \\
\hline PMMSE & Geometrical & Peli Malah Squared Error \\
\hline PFOM & Geometrical & Pratt's Figure Of Merit \\
\hline SODI & Geometrical & Odet's ODI \\
\hline SUDI & Geometrical & Odet's UDI \\
\hline ODI & Geometrical & Odet's ODI \\
\hline UDI & Geometrical & Odet's UDI \\
\hline PAD & Geometrical & Principal Axis Difference \\
\hline RMSSD & Geometrical & Root Mean Square Surface Distance \\
\hline
\end{tabular}

Table 5.2 - Attributes used for machine learning

The measures SODI, SUDI and BD receive parameters. For SUDI and SODI, a value of 2 has been chosen for the scaling factor $(\mathrm{n})$ and a value of 10 for the saturation factor $\left(\mathrm{d}_{\mathrm{TH}}\right)$. 
The scaling factor has been chosen to be more than 1 in order to put less emphasis on very small variations between the segmentation under test and reference segmentation boundaries as such small variations will not adversely affect this case study. For the same reason the $\mathrm{z}$ parameter in Baddeley's Distance has a value of 2.

Two categories of machine learning algorithms are explored in this thesis: decision trees and rule-induction techniques; the main motivation behind choosing these learning algorithms is that the classifiers built using these algorithms are very interpretable. This is important as experts will feel more confident in the predications made by these classifiers as they can vividly see the rules and decisions that lead to the predictions. Also our experiments show that more complex machine learning algorithms such as Support Vector Machines or Neural Networks do not perform any better in our application while not being as easy to interpret. An explanation of the classifiers we have used will follow in Section 5.3.

The average of 10 stratified 10 -fold cross-validations are used to validate the classifiers using the performance metrics: accuracy, kappa statistic and the area under the ROC curve. For a definition of cross-validation and these performance metrics please refer to Section 3.2 .

\subsection{Description of classifiers}

This section describes the three classification algorithms that are used in this thesis: J48, PART and JRIP. The readily available implementation of these classification algorithms in the WEKA (Waikato Environment for Knowledge Analysis) tool are used so the subtitle names below correspond to those names. Please refer to Section 5.8 for a description of the tools used in this case study.

\subsubsection{J48}

J48 implements the very well-established classification algorithm $\mathrm{C} 4.5$ that is the standard algorithm to create decision trees in both research and industry settings. 
As shown in Figure 5.5, a decision tree consists of attribute nodes, leaf nodes and decision branches. The attribute nodes or internal nodes are the nodes where a split is made based on the value of the attribute. If the attribute is nominal, one decision branch is made for each value of the nominal attribute. For numeric attributes, a splitting threshold is found which splits the training instances into two branches. The leaf nodes specify the class of the instances that follow the branches leading to them and terminate the growth of the tree.

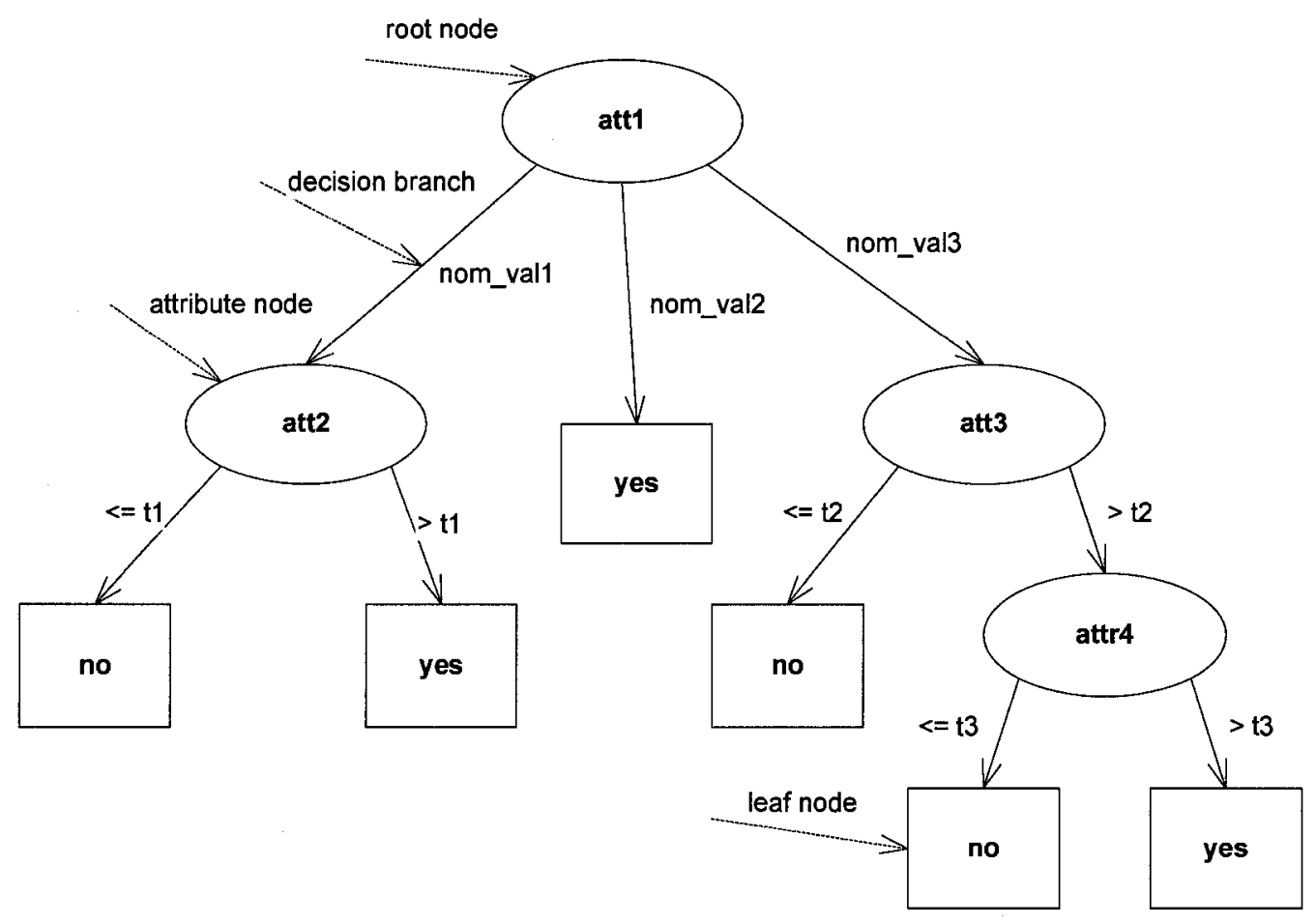

Figure 5.5 - A sample decision tree

To construct a decision tree, an attribute has to be selected to split on. The splitting attribute will divide the instances into different sets; one for each branch. If it is determined that a branch leads to a leaf node, the class of the instances in that path (i.e. consecutive branches) is known and the growth in that part of the tree is stopped, otherwise this procedure is continued recursively until all paths of the decision tree lead to a leaf node. $\mathrm{J} 48$ has a configurable parameter that restricts the minimum number of instances that are allowed to reach a leaf node. Choosing higher values for this parameter 
effectively prunes the tree (pruning is described a little further in this section), creating smaller trees but as the number of training instances is limited it may be better to allow the decision tree algorithm to decide how much to grow the tree rather than forcing it to stop by choosing a high value for the minimum number of instances per leaf configuration parameter.

The question remains on how to choose an attribute to split on. In information theory terms, an attribute is chosen such that a split on that attribute will require conveying the least number of information bits to determine the class of the training instances which in turn means that a split on that attribute leads to the maximum separation of the classes. This attribute is said to have divided the instance set into the purest subsets of instances. The entropy function defined in Equation 5.1 measures the extent of (im)purity of a set of instances for a binary-class problem.

Equation $5.1 \quad \operatorname{entropy}\left(p_{+}, p_{-}\right)=-p_{+} \log \left(p_{+}\right)-p_{-} \log \left(p_{.}\right)$

In Equation 5.1, $\mathrm{p}_{+}$and $\mathrm{p}_{\text {. }}$ are respectively the fraction of instances with class + and - in the instance set and the log is usually a base 2 logarithm. Entropy is measured in units of information bits. Note that in the two extreme cases, the entropy of a set of instances that only belong to one class is zero (completely pure instance set) and the entropy of a set of instances with an equal number of + and - instances is 1 (completely impure instance set). In any other case, the entropy of an instance set is a number between 0 and 1; higher values indicating the need for more information to be transmitted to distinguish the class of the instances. To align more with the concept of information transmission we define the info function (Equation 5.2) and use it instead of the entropy function as it is more intuitive. The info function is equivalent to the entropy function but it receives the number of positive and negative instances in an instance set instead of the fraction of them.

\section{Equation 5.2 $\operatorname{info}\left(n_{+}, n_{-}\right)=\operatorname{entropy}\left(p_{+}, p_{-}\right)$}

The information value of an attribute in any stage of the decision tree construction depends on the aggregate purity of the subsets which the attribute has split its input 
instances to and is defined in Equation 5.3. $\mathrm{n}$ is the number of input instances from the branch that leads to the attribute node. $n_{1+}$ and $n_{1-}$ are respectively the positive and negative instances in the left subset, similarly in the case of $n_{r^{+}}$and $n_{r^{-}}$for the right instances.

Equation 5.3

$$
\operatorname{info}(\text { attribute })=\frac{n_{l+}+n_{l-}}{n} \operatorname{info}\left(n_{l+}, n_{l-}\right)+\frac{n_{r+}+n_{r-}}{n} \operatorname{info}\left(n_{r+}, n_{r-}\right)
$$

The attribute that has reduced the info of the instance set the most is the one that is chosen for further splitting the instance set. The info gain shows exactly this (Equation 5.4):

\section{Equation 5.4}

$$
\text { info gain(attribute })=\text { info(attribute })- \text { info }\left(n_{+}, n_{-}\right)
$$

As an example, assume we have 14 training instances out of which 9 instances have class + and 5 instances have class -. Each instance is characterized by two nominal attributes each having only two values. We would like to choose the root node of the decision tree from these attributes. One attribute splits the instances such that the left branch has 3 instances of class + and 4 instances of class - while the right branch has 6 instances of class + and 1 instance of class -. The second attribute splits the instances such that the left branch has 6 instances of class + and 2 instances of class - while the right branch has 3 instances of class + and 3 instances of class -. The scenario is shown in Figure 5.6.
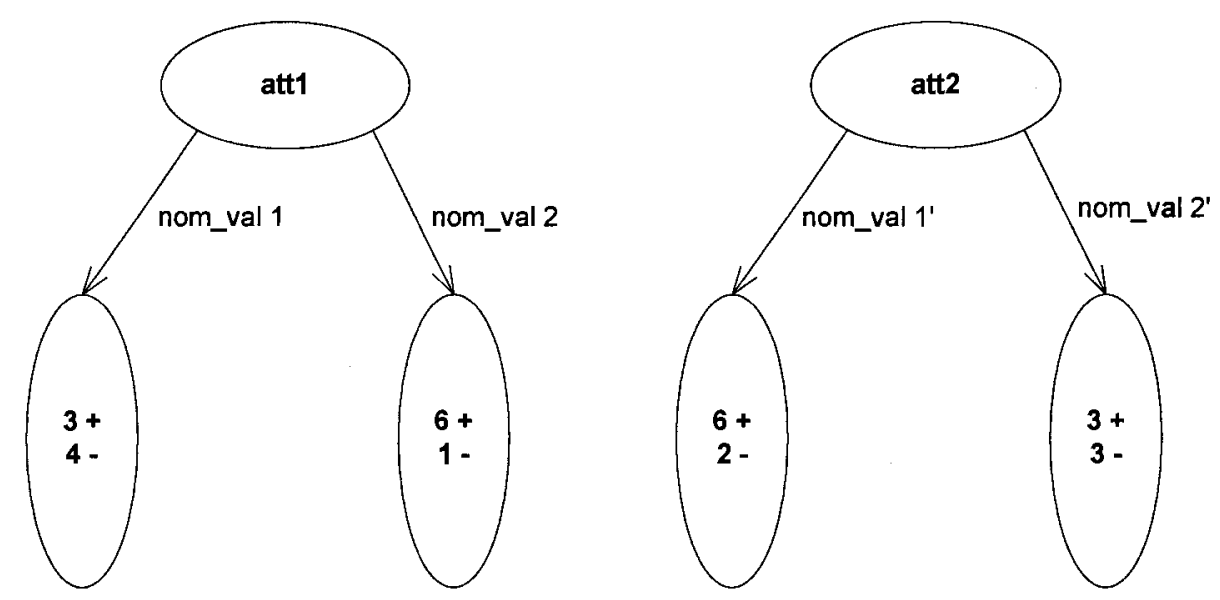

Figure 5.6 - Split of instances by attributes 1 and 2 
In order to find out which attribute to split on, we calculate the info gain of each attribute:

info gain(attribute 1$)=$ info(attribute1 $)-$ info $(9+, 5-)$

$=\frac{7}{14} \operatorname{info}(3+, 4-)+\frac{7}{14} \operatorname{info}(6+, 1-)-\operatorname{info}(9+, 5-)=0.971$

info gain(attribute2) $=$ info(attribute2) - info $(9+, 5-)$

$=\frac{8}{14} \operatorname{info}(6+, 2-)+\frac{6}{14} \operatorname{info}(3+, 3-)-\operatorname{info}(9+, 5-)=0.020$

As attribute 1 reduces the information bits required for determining the class of the instances by a much larger value then attribute 2 , attribute 1 is chosen as the root node.

In C4.5 the gain ratio is used instead of the info gain as the criteria for choosing the attributes to split on. The gain ratio is calculated by dividing the info gain of the attribute by its info. Further discussion as to why the gain ratio is used is out of the context of this thesis.

The values of the decision branches are known when using nominal values. An attribute node with a nominal attribute simply divides the instances that reach it to as many subsets as the number of values the nominal attribute accepts. In the case of numeric attributes (the case in this thesis) the decision branches are not readily known. Assuming we only use binary splits for numeric attributes, we have to find a way to split the instances that reach the numeric attribute node. This is done by using the info gain again i.e. in the most crude way every split point possible between the attribute values of the instances that reach the attribute node are considered and the info gain of the attribute resulting from the splits are calculated. The split that results in the highest info gain is chosen to be the decision criteria for the branches. Further discussion of optimizing this process is out of the context of this thesis.

A common problem in any machine learning algorithm is learning a classifier that is over-fitted to the training data and cannot perform well when classifying unknown instances. To avoid this, similar to pruning a tree, we need to prune the classifier. Usually the pruning process takes place after the classifier has been built. The most 
familiar pruning method in decision trees is subtree replacement where a subtree is replaced by a leaf node. Other operations such as subtree raising are also used in C4.5 which we will not delve into more. A decision criterion has to be found to evaluate whether this operation will actually improve the performance of the tree. To do so the error rate of the tree before and after the subtree replacement operation is calculated and if the error rate decreases the subtree is replaced by the majority class leaf node. If we use the training data to calculate the error rate no pruning will ever take place as the error rate will always increase after the pruning operation [24]. There are two ways to tackle this problem.

One method is take a similar approach as cross-validation and divide the training data into a number of folds using one fold for pruning and the other folds for training the classifier. This is the approach taken in reduced-error pruning. In this way we make the decision of whether to replace a subtree or not by comparing the error rate on the pruning set which is independent of the training set. This approach has the obvious drawback of reducing the number of instances used to train the data.

Another method is to use the training data, but this time instead of using the error rate that is obtained from the classification of the pruning set before and after pruning, we define our own error rate. The error rate here is the error that is introduced by replacing a node with the majority class of the instances that reach that node i.e. the ratio of misclassified instances as a result of the replacement to the total number of instances that reach that node. As we are using the training instances, this error rate may be too optimistic. Using the Bernoulli process to model the true error rate, we use the upper confidence limit of the true error rate as our decision criteria for replacing a node which takes away the optimism. We denote this error estimate as the $\mathrm{C} 4.5$ error estimate. To use it, we first calculate the $\mathrm{C} 4.5$ error estimate for a parent node and then compare it with the combined C4.5 error estimates of its leaf nodes (the leaf node error estimates are combined in proportion with the ratio of the number of instances that reach each of them). If the parent node $\mathrm{C} 4.5$ error estimate is lower, then pruning will take place. This approach to pruning is proven to be effective in practice and is the default method of pruning for the $\mathrm{C} 4.5$ classifier. We use $\mathrm{C} 4.5$ pruning for training the $\mathrm{J} 48$ classifier. 


\subsubsection{PART}

PART is a rule-induction algorithm. Rule-induction algorithms take a covering approach towards learning the classifier. In this approach, an attempt is made to construct a rule that covers all the instances. Rule construction for the uncovered instances is continued recursively until all the instances are covered by a rule.

PART uses partial decision trees to construct rules. It constructs a rule from the branch leading to the leaf node that covers the most instances, thus creating the most general rule. PART tries to avoid building a full decision tree for each rule. To do so, for every attribute split, PART sorts the resulting subsets in increasing order of their entropy, starting to grow the instances of the subset with the least entropy (unless the entropy is zero which would make the subset a leaf). This is because the subsets with lower entropies lead to smaller sub-trees and more generic rules as opposed to the subsets with high entropies which need to be grown more in order to reach the leaf nodes. The lowest entropy subset is grown in a recursive manner until leaf nodes are reached at which stage PART immediately considers the option of pruning the attribute node that branches only to leaf nodes. If pruning takes place, the attribute node is replaced with the majority class leaf node and backtracking is done to investigate further options for pruning. In the case where it is decided against pruning, the tree growth stops at this point and the non-grown subsets are left unexplored. This creates a partial decision tree where not all the branches lead to leaf nodes. As mentioned before a rule is constructed from the branch that leads to the majority class leaf node. PART uses the same configuration parameters as J48 because it is building decision trees with the same heuristics as C4.5. We use C4.5 pruning for PART too.

\subsubsection{JRIP}

JRIP is also a rule-induction algorithm. JRIP implements the RIPPER (Repeated Incremental Pruning to Produce Error Reduction) algorithm [25] which is an industrystrength rule-induction algorithm. For each class, JRIP tries to find the rule that covers most of the instances and at the same time has the best success rate. This procedure is repeated recursively for the uncovered instances until a set of rules are constructed that 
cover all the instances for that class. The same pattern is repeated for every class. Immediately after each rule is constructed, pruning takes place where different tests from the rule is deleted and a heuristic is used to evaluate if the pruned rule is better than the original rule. More tests are deleted from the rule until the heuristic predicts worse performance where the pruning process stops for that rule. To do the pruning, the training instances are split into a growing set (used for training the classifier) and a pruning set (used for pruning the rules), which means that the original rule is trained using less instances than would be available if we did not perform any pruning. We have used $1 / 3$ of the training instances for pruning and the rest for training the rules. This approach where we prune the rule immediately after its construction is referred to as incremental reduced-error pruning.

The RIPPER algorithm further improves the final rule set by performing a global optimization stage after the rules are constructed. In this stage, for each rule, two variants are constructed and if any of the two variants perform better they are used instead of that rule. Further details on how the performance of these variants is compared with the original rule are out of the context of this study. Please refer to [25] for more information. JRIP allows the optimization step to be repeated for a user-defined number of times. In the case study we have used 4 optimization stages for building the JRIP classifier.

\subsection{Attribute correlations}

The correlation between two random variables determines whether the two variables have a tendency to increase and decrease together. One statistical measure used for this purpose is Spearman's rank correlation coefficient.

Equation 5.5 $r_{S}=\frac{\sum_{i=1}^{n}\left(R\left(X_{i}\right)-\frac{n+1}{2}\right)\left(R\left(Y_{i}\right)-\frac{n+1}{2}\right)}{\frac{n\left(n^{2}-1\right)}{12}}$

In Equation 5.5 [28], $r_{S}$ is the Spearman coefficient between the random variables $\mathrm{X}$ and Y.R $\left(X_{i}\right)$ and $R\left(Y_{i}\right)$ associate a rank to the variables $X_{i}$ and $Y_{i}$ respectively. The rank is 
simply the order of each of the values that the variables represent, when the variable values are ordered from smallest to largest.

The Spearman coefficient has some advantages over its equivalent correlation coefficient i.e. the Pearson correlation coefficient. It does not assume any normal distribution for the variables and is not as sensitive to outliers in the data which are the main reasons that this particular statistical measure has been used in this thesis. Table 5.3 shows the Spearman coefficient between the pairs of attributes that are highly correlated (Spearman coefficient more than or equal to 0.9 ).

Table 5-3 is very consistent with the definition of the measures in Section 3.1. TC and DSC are both trying to capture the common area between the True Segmentation (TS) and the Segmentation Under Test (SUT) which makes them highly correlated. SODI and SUDI (with parameters $\mathrm{n}=2$ and $\mathrm{d}_{\mathrm{TH}}=10$ ) prove to be very highly correlated to their non-scalable counterparts. The only difference between PMME and PMMSE is that PMME sums the distances from the SUT's boundary pixels to the TS boundary pixels while PMMSE sums the square of the distances, making these two variables very highly correlated. Baddeley's Distance measure is an average of Hausdorff's measure again making these two measures highly correlated.

\begin{tabular}{|l|l|l|}
\hline TC & DSC & 1.000 \\
\hline SODI & ODI & 0.995 \\
\hline SUDI & UDI & 0.992 \\
\hline HD & BD & 0.961 \\
\hline PMME & PMMSE & 0.955 \\
\hline
\end{tabular}

Table 5.3 - Spearman's rank correlation coefficient between highly correlated attribute pairs

An attribute that is highly correlated to another attribute tends not to add any discriminating power (in terms of learning the class of the instances) during the classifier 
learning process. In the case where many correlated attributes exist it may be better to remove some of them to reduce the classifier construction time. In our case as the number of the attributes is still not a significant number we let the classifier decide which attributes to choose and also attempt to filter the attributes using filters and wrappers (Section 5.5).

\subsection{Attribute sets}

To compare the performance of the different classifiers, 9 different attribute sets have been considered (Table 5.4) to teach the machine learning algorithms.

\begin{tabular}{|c|c|c|c|}
\hline & Attribute Set & Selection Criteria & \\
\hline 1 & TC-DSC-TPVF-FPVF-AVD-ANVD & Overlap and volume difference measures & OV \\
\hline 2 & TC-DSC-AVD-ANVD & $\begin{array}{l}\text { Overlap and volume difference measures } \\
\text { chosen by the CFS filter using exhaustive } \\
\text { search }\end{array}$ & OVC \\
\hline 3 & TC-FPVF & $\begin{array}{l}\text { Overlap and volume difference measures } \\
\text { chosen by the J } 48 \text { decision tree wrapper using } \\
\text { forward selection greedy search }\end{array}$ & ovw \\
\hline 4 & $\begin{array}{l}\text { BD-HD-PFOM-RMSSD-ADBD-SODI- } \\
\text { ODI-SUDI-UDI-PAD-PMME-PMMSE }\end{array}$ & Geometrical measures & G \\
\hline 5 & HD-BD-PFOM-RMSSD-ODI-UDI-PAD & $\begin{array}{l}\text { Geometrical measures chosen by the CFS filter } \\
\text { using exhaustive search }\end{array}$ & $\mathrm{GC}$ \\
\hline 6 & PFOM-SODI-SUDI & $\begin{array}{l}\text { Geometrical measures chosen by the J48 } \\
\text { decision tree wrapper using forward selection } \\
\text { greedy search }\end{array}$ & GW \\
\hline 7 & $\begin{array}{l}\text { TC-DSC-TPVF-FPVF-AVD-ANVD-BD- } \\
\text { HD-PFOM-RMSSD-ADBD-SODI-ODI- } \\
\text { SUDI-UDI-PAD-PMME-PMMSE }\end{array}$ & All measures & A \\
\hline 8 & $\begin{array}{l}\text { TC-DSC-AVD-ANVD-HD-RMSSD-ODI- } \\
\text { UDI }\end{array}$ & $\begin{array}{l}\text { All measures chosen by the CFS filter using } \\
\text { exhaustive search }\end{array}$ & $\mathrm{AC}$ \\
\hline 9 & TC-FPVF-SODI & $\begin{array}{l}\text { All measures chosen by the J48 decision tree } \\
\text { wrapper using forward selection greedy search }\end{array}$ & AW \\
\hline
\end{tabular}

Table 5.4 - Attribute sets and their selection criteria

Three categories of measures have been chosen as the base attribute sets for training the classifiers: overlap and volume difference, geometrical and all the measures. We want to see how well the different types of comparison measures would do standalone and what 
would be the effect of combining these measures. The overlap and volume difference measures have been combined together. These measures convey less information regarding the differences between the two segmentations (specifically do not consider the shape differences) and have less complexity in terms of implementation compared to the geometrical measures. We may refer to these measures as simple measures.

In order to investigate how the classifier performance would change when built using a filtered set of attributes we have applied the Correlation-based Feature Selection (CFS) filter and a J48 based wrapper to the three main categories of attribute sets creating 6 more attribute sets.

Filters and wrappers attempt at taking out attributes that do not add any significant improvement in building a better classifier; also taking into consideration the association between the instance attributes and the instance class in their decision making (supervised attribute selection methods). The main motivation for choosing a wrapper and CFS filter were the benchmarking results of different attribute selection methods in reference [26].

Correlation-based Feature Selection (CFS) chooses a subset of attributes from the original attribute set that have a high correlation with the class and a low correlation with each other. Wrappers select attributes by first training a classifier from different subsets of the original attribute set and choosing the subset of attributes that trains the best performing classifier.

Filters and wrappers all require searching the subset space applying their evaluation criteria to each subset and choosing the best one. Choosing an exhaustive search method may seem to be the best option at all times but as the number of attributes goes up the search time for all the subsets also increases. Considering that the evaluation criteria have to also be applied to all the found subsets using an exhaustive search method may seem prohibitive. This specially comes into picture when choosing a wrapper method where a classifier has to be built for every subset found. For this reason, greedy search methods such as forward or backward hill climbing search are used to search the attribute space for subsets. In a forward hill climbing search method, we start from an empty subset and check the evaluation criteria to find the best single attribute. In the next stage, we add 
another attribute to the selected attribute and find the best pair of attributes. This process continues until the addition of no attribute will result in a performance improvement at which point the current subset is the selected subset of attributes. Backward selection takes a similar approach but starts off from the complete set of attributes and removes an attribute at each stage.

As described in Table 5.4, we have chosen the exhaustive search method for the CFS filter and forward selection hill climbing for the J48 wrapper method.

\subsection{Classifiers}

This section describes the output models (classifiers) of the different classification algorithms (J48, PART, JRIP) and explains how to interpret the output models.

\subsubsection{J48}

To train the J48 classifier the parameters in Table 5.5 are used. The number of instances per leaf node has been chosen after sweeping this parameter from 2 to 10 and finding that 4 instances per leaf is either significantly better or equivalent to the other values in the majority of the cases when using the attribute sets in Table 5.4 to train the J48 classifier. The comparison criterion is accuracy. Also we see that $\mathrm{C} 4.5$ pruning results in either a significantly better or equivalent result to reduced error pruning which is the motivation behind preferring $\mathrm{C} 4.5$ pruning over reduced error pruning.

\begin{tabular}{|l|l|}
\hline Parameter & Value \\
\hline Number of instances per leaf node & 4 \\
\hline Pruning method & C4.5 pruning \\
\hline
\end{tabular}

Table 5.5 - J48 configuration parameters

To evaluate significance the standard paired t-test (Equation 5.6) has been used. In Equation 5.6, $t$ has a student's $t$ distribution with $\mathrm{n}-1$ degrees of freedom (the student's $\mathrm{t}$ 
distribution tends to a normal distribution for larger degrees of freedom). $\bar{d}=\bar{X}-\bar{Y}$, where $\mathrm{X}$ and $\mathrm{Y}$ are two random samples with $\mathrm{n}$ values and $\sigma_{d}^{2}$ is the variance of $\bar{d}$. The $\mathrm{t}$-test assumes that the mean follows a normal distribution regardless of the distribution of the samples themselves which is a valid assumption if we have enough samples. In our case, the t-test has been performed on the mean of the accuracies that have been obtained after performing 10 times stratified 10 fold cross validation creating a sample size of 100 (a reasonable number of samples).

Equation 5.6 $\quad t=\frac{\bar{d}}{\sqrt{\frac{\sigma_{d}^{2}}{n}}}$

The paired t-test is a standard statistical test used to evaluate whether the null hypothesis specifying that two random samples have the same mean can be rejected or not. If rejected, this would indicate that the difference between the means is significantly different from each other. In order to reject the null hypothesis, the calculated $t$ value must lie out of the bounds of the confidence limits associated with the chosen significance level. A significance level of $5 \%$ indicates that with a confidence factor of $95 \%$ we can conclude that the null hypothesis is rejected or accepted. As it is not obvious whether $\bar{X}$ is greater than or smaller than $\bar{Y}$, we use a two-tailed test.

Figure 5.7 shows the J48 classifier created by only using the simple measures (overlap and volume difference measures), before and after applying a wrapper to the attribute set. We will see in Section 5.7.3 that training the classifier with only the wrapper selected attribute set performs significantly better than just using the original attribute set, thus we have shown both classifiers for the sakes of comparison. As we will show the wrapper performs better for the other two attribute set categories too (GW versus $\mathrm{G}$ and $\mathrm{AW}$ versus A). As the CFS filter tends to perform significantly worse than the original attribute set we have not included the trained classifiers by the CFS filtered attribute sets.

In order to interpret the classifiers, we will use the term segmentation under test to refer to the segmentation output by the current iteration of the segmentation algorithm and the 
term reference segmentation for the segmentation that it is being compared to from the previous revisions of the segmentation algorithm.

The decision tree shown in Figure 5.7 (a) shows if the overlap (denoted by TC, as opposed to DSC) between the two segmentations is less than approximately $75 \%$ they are considered consistent, otherwise if AVD indicates that the calculated volume for the left ventricle depicted by the segmentation under test is not more than $5 \mathrm{~cm}^{3}$ larger than the volume associated with the reference segmentation the two segmentations are considered to be consistent. This indicates that if the volume difference is more than $5 \mathrm{~cm}^{3}$ then we need more information to determine the consistency between the two segmentations. FPVF provides this extra information. If the segmentation under test has less than $0.56 \%$ extra labeled pixels out of all the pixels that are not labeled by the reference segmentation then we have a consistent pair again, while labeling more pixels not labeled by the reference segmentation will yield an inconsistent result. Note here the lack of any information about the relative shape difference of the extra labeled pixels in the segmentation under test with respect to the reference segmentation. This information is not present in simple measures such as FPVF.

As the volume difference measures are eliminated from the attribute set after they pass through the wrapper (Table 5.2), the instances with the reduced attribute set train a classifier that only uses the overlap measures (Figure 5.7 (b)). As shown in this tree, two segmentations may still be consistent if FPVF is more than $0.33 \%$ and they are only inconsistent if FPVF is more than $0.56 \%$. This result matches what we see in Figure 5.7 (a). 

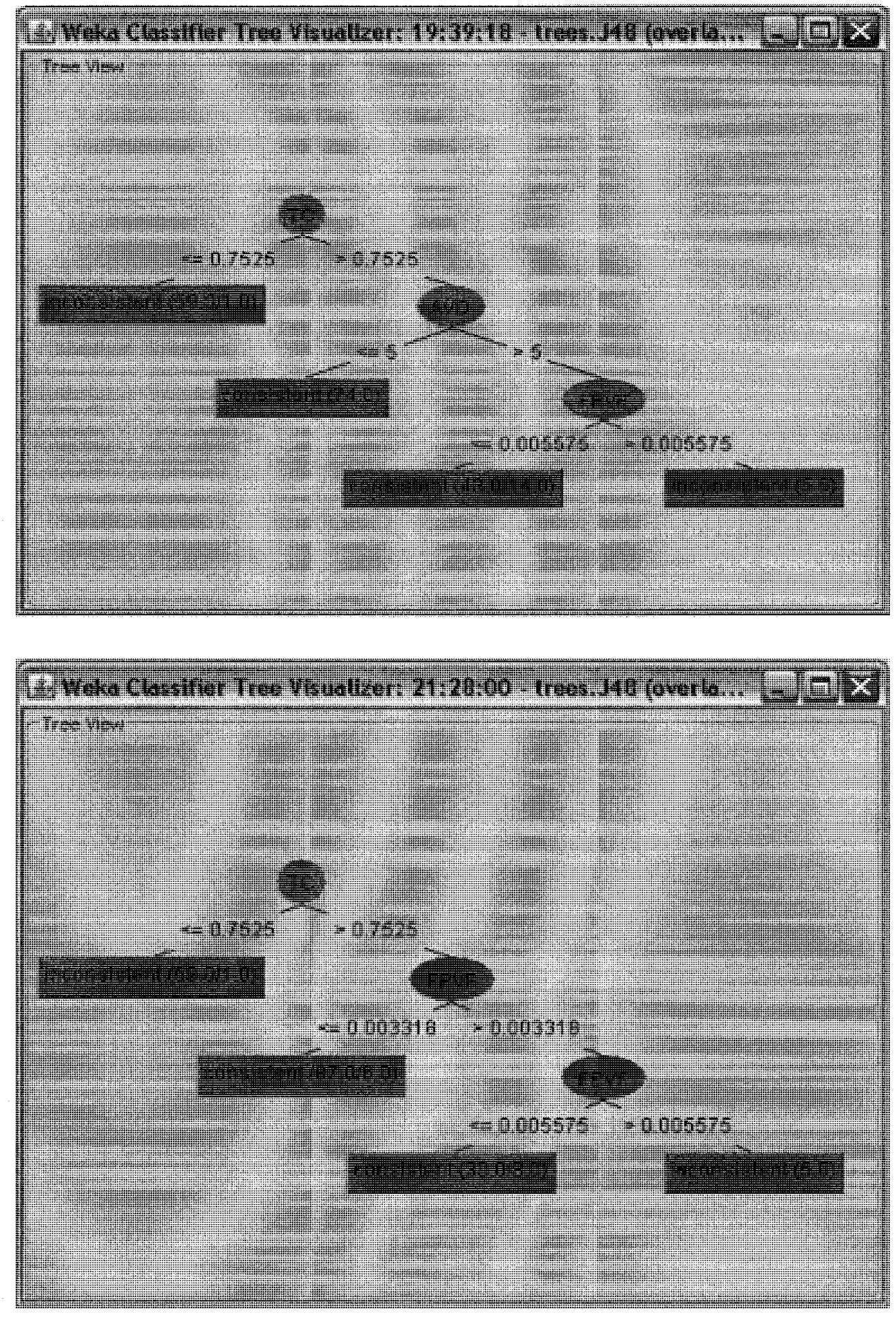

Figure 5.7 - (a) Top: J48 classifier trained with simple measures (OV) (b) Bottom: J48 classifier trained with the simple measures selected using J48 wrapper (OVW) 
The numbers in the parentheses respectively (from left to right) represent the number of training instances that reach that leaf node and the number of these instances that are incorrectly classified by that leaf node. The incorrect classifications are a result of pruning; otherwise we would have a very over-fitted tree to the training data that would not perform well on unknown instances. Paths that reach leaf nodes with less misclassified instances are more reliable paths i.e. we have more confidence in the prediction of the decision tree if our unknown instance is classified by the leaf node in these paths. Also note that a leaf cannot be created with less than 4 instances in that leaf as per our configuration parameters (Table 5.5).

Figure 5.8 shows the classifiers trained by geometrical measures, before and after applying a wrapper. Baddeley's distance has been preferred to its more noise-sensitive counterpart, Hausdorff's distance and is the main discriminating factor for classifying the negative instances, determining that $66 \%$ (51 out of 77) of the negative instances (inconsistent pairs) have a Baddeley distance of more than 16.73. We also see 92 out of the 104 positive instances (consistent pairs) do not exceed a Baddeley distance of 16.73 and an ODI distance of 2.10 i.e. $88 \%$ of the consistent pairs in the training dataset. We observe in this classifier that the split made by UDI is somewhat confusing as you would usually expect more inconsistency when UDI has a larger value as opposed to a smaller one. This split just means that we have 4 inconsistent pairs in the training set with a UDI of less than 1.67 and 6 consistent pairs with a UDI of more than 1.67. This may indicate that using UDI by itself may not be a good indicator of the consistency between two segmentations, also confirming that using many measures in conjunction with each other is more effective in classifying consistent or inconsistent pairs rather than only using one measure to predict consistency. For example, if our training set is biased towards segmentations with a lot of oversegmentation, then UDI will not be a good performer as it only considers the distance of the undersegmented pixels to the reference boundary. These kind of attribute splits will be much more unlikely upon availability of larger training sets with more representative instances for each class. 

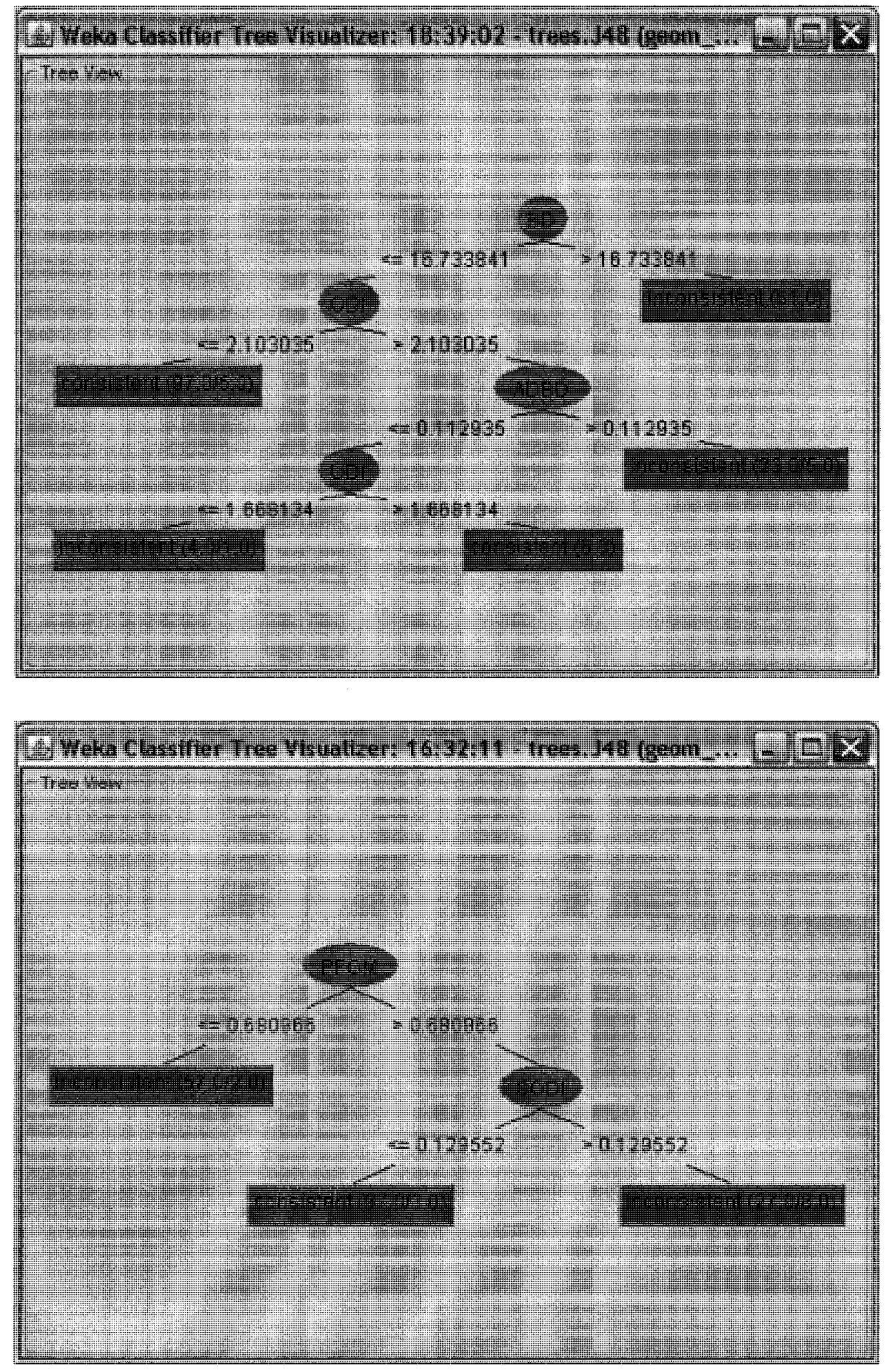

Figure 5.8 - (a) Top: J48 classifier trained with geometrical measures (G) (b) Bottom: J48 classifier trained with the geometrical measures selected using $\mathbf{J 4 8}$ wrapper (GW) 
The J48 wrapper does not see Baddeley's Distance (BD) as necessary for training the classifier omitting it from the attribute set, this is while all the attributes selected in the tree of Figure 5.8 (a) are chosen again. This time Pratt's Figure of Merit finds 55 out of 77 inconsistent pairs ( $71 \%$ ) have a PFOM of less or equal to 0.68 . Odet's scalable version of ODI which we refer to as SODI is chosen instead of ODI (even though the choice remains to pick any of these as both attributes are chosen by the wrapper selected attribute set) resulting in a normalized discriminating factor. 94 of the 104 consistent pairs have a PFOM of more than 0.68 but a SODI of no more than 0.13.This indicates $90 \%$ of the consistent pairs in the training dataset can be classified correctly by only using PFOM and SODI. Notice how the size (number of nodes in the tree) of the tree trained by only the wrapper selected attributes has reduced by 4 (from 9 to 5) and the length (number of node levels of the tree) of the tree by 2 (from 5 to 3 ). We will see in Section 5.7.3 that the simpler tree actually results in better performance. This may be because the simpler trees are less overfitted to the training instances.

Using all the measures to train the classifiers, results in the trees shown in Figure 5.9. Figure 5.9(a) is trained with all the measures and Figure 5.9 (b) is trained with only the ones chosen by the wrapper. Similar to when only using the simple measures the wrapper has removed the volume difference measure AVD and replaced it with FPVF. This proves to be the right choice as we will see in Section 5.7.3 based on the cross-validation results. We see in both cases that the overlap measure, TC and the geometrical measure, SODI are chosen as the main discriminating factors for classifying the instances. Also notice that both trees are quite simple and not lengthy or bushy (with many leaves). 

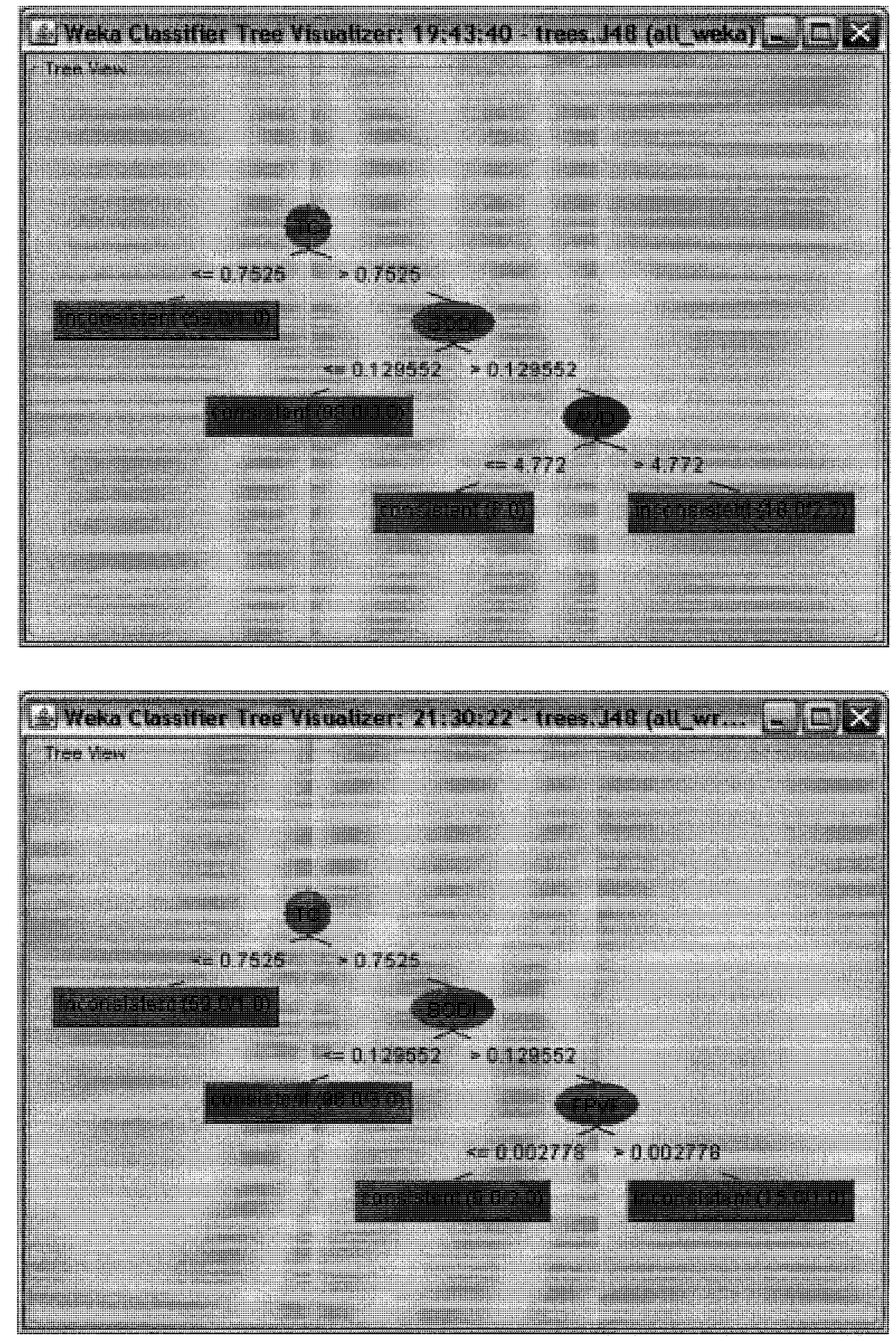

Figure 5.9 - (a) Top: J48 classifier trained with all comparison measures (A) (b) Bottom: $\mathrm{J48}$ classifier trained with the comparison measures selected using $\mathrm{J48}$ wrapper (AW) 


\subsubsection{PART}

We will show what kind of rules PART classifiers use to classify instances. The approach would be similar to Section 5.6.1. We will explore the classifiers produced by PART, both before and after applying the wrapper for all three main attribute set categories: simple, geometrical and all attributes. The parameters used for configuring the PART classification algorithm are the same as J48, as PART uses partial decision trees as its underlying mechanism for generating rules (5.3.2). We find that the same values that were used for J48 (Table 5.5) are also a better choice for PART with the same justification used for J48 (first paragraph of Section 5.6.1).

PART generates the following rules when the training instances are only identified by the overlap and volume difference measure values:

1) If $((\mathrm{TC}>0.7525) \& \&(\mathrm{AVD}<=5))$ then class $=$ consistent $(74)$

2) Else if (ANVD > 0.204632) then class = inconsistent (54)

3) Else if (FPVF $<0.005575)$ then class $=$ consistent $(48 / 18)$

4) Else class $=$ inconsistent (5)

4 rules are generated. The first rule covers 74 of the positive instances and can be directly deduced from the equivalent decision tree (Figure 5.7 (a)). In addition to using AVD in the first rule, PART also uses the normalized volume difference measure, the Absolute Nominal Volume Difference (ANVD), in the second rule to cover 54 of the negative instances. The third rule has a $37.5 \%$ error rate (18 out of the 48 instances that are covered by that rule are incorrectly classified) which makes the consistent prediction for the unknown instances that are covered by this rule somewhat questionable. These are instances which are not covered by the first two rules.

The classifier trained by the wrapper selected attribute set is as follows:

1) If $((\mathrm{TC}>0.7525) \& \&(\mathrm{FPVF}<=0.003318))$ then class $=$ consistent $(87 / 6)$ 
2) Else if $(\mathrm{TC}<0.827986)$ then class $=$ inconsistent $(66 / 2)$

3) Else class $=$ consistent $(28 / 7)$

Again the first rule can be easily deducted from the equivalent decision tree (Figure 5.7 (b)). Similar to Figure 5.7 (b) TC is the main discriminator for covering the negative instances but here the threshold is higher $(0.83$ versus 0.75$)$ covering more inconsistent pairs (64 versus 58$)$.

Using geometrical measures we find 2 rules generated by PART:

1) If $((\mathrm{BD}<=16.733841) \& \&(\mathrm{ODI}<=2.103035)$ then class $=$ consistent $(97 / 5)$

2) Else class $=$ inconsistent $(84 / 12)$

The rules generated after feeding the wrapper selected attributes to PART:

1) If $((\mathrm{PFOM}>0.680966) \& \&(\mathrm{SODI}<=0.129552))$ then class $=$ consistent $(97 / 3)$

2) Else class $=$ inconsistent $(84 / 10)$

Again we see how in many cases the rules generated by PART can be easily deducted by following certain paths in the equivalent decision trees (the rule that predicts consistent pairs is one path of the decision trees trained with the same attribute set). This makes sense as PART recursively covers the training instances by constructing a partial J48 decision tree (as described in Section 5.3.2) for every rule and making the leaf node covering the largest number of instances a rule. The wrapper trained PART classifier (using only geometrical measures) above is identical to its equivalent decision tree as if either SODI is less than 0.13 or PFOM is less than 0.68 then the pair is predicted to be inconsistent which is exactly what its equivalent decision tree (Figure 5.8(b)) is predicting. Notice the number of training instances that have incorrectly been classified by this rule is also the same i.e. 10. The decision tree further clarifies that 2 of these where misclassified by the condition (PFOM $<0.68$ ) and the remaining 8 by the condition (SODI < 0.13). 
Using all measures we get the following classifier:

1) If $((\mathrm{TC}>0.7525) \& \&(\mathrm{SODI}<=0.129552))$ then class $=$ consistent $(98 / 3)$

2) Else if $(\mathrm{AVD}>8.272)$ then class = inconsistent $(69 / 1)$

3) Else if $(\mathrm{ANVD}<=0.138212)$ then class = consistent $(10 / 2)$

4) Else class $=$ inconsistent (4)

After applying the wrapper the following four rules are generated:

1) If $((\mathrm{TC}>0.7525) \& \&(\mathrm{SODI}<=0.129552))$ then class $=$ consistent $(98 / 3)$

2) Else if $(\mathrm{TC}<=0.76311)$ then class $=$ inconsistent $(59 / 1)$

3) Else if (FPVF $>0.002778)$ then class $=$ inconsistent $(15 / 1)$

4) Else class $=$ consistent $(9 / 2)$

This classifier is a replica of the tree trained with the equivalent training set (Figure 5.9 (b)) with a slight difference in the threshold used for TC for identifying inconsistent pairs ( 0.76311 in the second rule versus 0.7525 in the decision tree). This initiates from the fact that PART constructs a different decision tree to generate the second rule which means that it may not necessarily come up with the same threshold as the first rule when trying to cover the remaining instances (the instances that are not covered by the first rule).

\subsubsection{JRIP}

We will take the same approach as Sections 5.6.1 and 5.6.2 to analyze the classifiers constructed by JRIP. The main two configuration parameters for JRIP are outlined in Table 5.6. A value of 3 for the number of folds indicates that JRIP will use $2 / 3$ of the training set for constructing the rules and $1 / 3$ for pruning them. 4 iterations of optimizations will follow when all the rules are constructed. For more information about these parameters please refer to Section 5.3.3. Sweeping the number of folds from 2 to 10 and the global optimization runs from 2 to 10 shows that the chosen values for these 
configuration parameters results in either a significantly better or equivalent classifier in the majority of cases when tested with the t-test using accuracy as the performance metric.

\begin{tabular}{|l|l|}
\hline Parameter & Value \\
\hline Number of folds & 3 \\
\hline Number of global optimizations & 4 \\
\hline
\end{tabular}

Table 5.6 - Configuration parameters for JRIP

The rules generated by JRIP when fed with training instances using different attribute sets are as follows:

Using only simple measures:

1) If ( $\mathrm{TC}<=0.7525)$ then class $=$ inconsistent $(59 / 1)$

2) Else if $((\mathrm{AVD}>=9.169) \& \&(\mathrm{FPVF}>=0.00333) \& \&(\mathrm{TC}<=0.867675))$ then class $=$ inconsistent (12/1)

3) Else consistency $=$ consistent $(110 / 8)$

Simple measures that have gone through the J48 wrapper:

1) If $(\mathrm{TC}<=0.7525)$ then class = inconsistent $(59 / 1)$

2) Else if $(\mathrm{FPVF}>=0.005522)$ then class $=$ inconsistent $(7 / 1)$

3) Else class $=$ consistent $(115 / 13)$

Geometrical measures:

1) If $((\mathrm{BD}>=10.600194) \& \&(\mathrm{BD}>=17.571708))$ then class $=$ inconsistent $(51 / 0)$

2) Else if $((\mathrm{ODI}>=2.144941) \& \&(\mathrm{ADBD}>=0.113931))$ then class $=$ inconsistent $(23 / 5)$ 
3) Else if (SUDI $<=0.016691)$ then class $=$ inconsistent $(4 / 1)$

4) Else class $=$ consistent $(103 / 5)$

We see here that the first condition (BD $>=10.6)$ in the first rule can be omitted without any difference in the classifier performance. This indicates that even more pruning is required here. The first two rules are equivalent to different paths of the corresponding decision tree (Figure 5.8(a)) with minor modifications in the thresholds i.e. 17.57 for BD as opposed to 16.73 (although they both find 51 inconsistent pairs) and very slight differences (in the order of a thousandth of a digit) for ODI and ADBD which is insignificant in this context, as we see again that the number of instances that are categorized by these conditions is the same as the decision tree ( 23 instances out of which 5 are incorrectly classified as inconsistent).

Geometrical measures that have gone through the J48 wrapper:

1) If $((\mathrm{PFOM}<=0.680966)$ then class $=$ inconsistent $(57 / 2)$

2) Else if $((\mathrm{SODI}>=0.131275)$ then class $=$ inconsistent $(27 / 8)$

3) Else class $=$ consistent $(97 / 3)$

\section{All measures:}

1) If $(\mathrm{ANVD}>=0.148995) \& \&(\mathrm{RMSSD}>=4.357064)$ then class $=$ inconsistent $(60 / 0)$

2) Else if $((\mathrm{SODI}>=0.131275) \& \&(\mathrm{AVD}>=5.061))$ then class $=$ inconsistent $(15 / 2)$

3) Else class $=$ consistent $(106 / 4)$

Notice how the combination of ANVD and RMSSD predict 78\% of the inconsistent pairs with zero error rate. JRIP uses different measures compared to its equivalent J48 and PART classifiers here, not taking advantage of TC at all for finding inconsistent pairs. Cross-validation results will indicate whether JRIP has constructed a better classifier compared to J48 and PART or not (Section 5.7.2). 
All measures that have gone through the J48 wrapper:

1) If $(\mathrm{TC}<=0.737932)$ then class $=$ inconsistent $(58 / 1)$

2) Else if $((\operatorname{SODI}>=0.131275) \& \&(\mathrm{FPVF}>=0.002838))$ then class=inconsistent $(16 / 1)$

3) Else class $=$ consistent $(107 / 5)$

We do not directly see a rule indicating that segmentation pairs with TC more than 0.75 (here 0.74 ) and SODI less than 0.13 are mainly consistent (comparing with Figure 5.9 (b) or the equivalent PART rules) but it can be indirectly deduced.

Looking at the classifiers generated by JRIP we see some noticable points:

1) The rules generated by PART show more resemblance to different paths in the decision tree that is trained with the same instances and attribute set than JRIP.This is due to the fact that JRIP does not use any decision tree constructs in its rule generation scheme whle PART uses partial decision trees.

2) JRIP covers all the instances that belong to one class before going to the next class (in all the JRIP classifiers above, first rules have been generated to cover the inconsistent class and then the consistent class, while this is not the case for PART). This is due to the nature of the RIPPER rule induction algorithm. PART does not behave this way as it uses partial decision trees for its rule induction, and depending on the chosen leaf at each stage instances belonging to a different class may be covered.

\subsubsection{Conclusion}

An investigation of the measures used in the classifiers constructed by the three different classification algorithms shows: 
1) TC and FPVF are the most popular overlap measures. TC was preferred to DSC which is very highly correlated to it (Table 5.3). Also TPVF was never chosen.

2) SODI is the most popular geometrical measure being used in close to all the classifiers that have geometrical measures involved in training them.

3) Good discriminators for negative instances (inconsistent pairs) are:

a. Using only simple measures: TC

b. Using only geometrical measures: $\mathrm{BD}, \mathrm{PFOM}$

c. Using all measures: TC and the combination of ANVD and RMSSD as discovered by JRIP.

$78 \%$ (using only simple measures) to $91 \%$ (using all measures) of the negative instances are covered with the above measures.

4) Good discriminators for positive instances (consistent pairs) are the following combinations:

a. Using only simple measures: TC and AVD or TC and FPVF.

b. Using only geometrical measures: BD and ODI or PFOM and SODI.

c. Using all measures: TC and SODI.

Around $75 \%$ of the positive instances are covered by the above combinations.

5) When using all the measures, TC was the main discriminator for negative instances and the combination of TC and SODI was the main discriminator for the positive instances. If TC determines that the two segmentations have a less than $75 \%$ overlap, then the two segmentations are inconsistent. Otherwise if SODI determines that the average oversegmented pixels have a normalized distance of less than 0.13 to the boundary of the reference segmentation then 
the two segmentations are consistent and thus diagnostically equivalent. This is specially evident in the case of $\mathrm{J} 48$ and PART.

\subsection{Classifier Performance Comparison}

In this section we will analyze the performance of the classifiers constructed by the attribute sets outlined in Table 5.4. The performance metrics used for comparing the classifiers are: accuracy, Kappa statistic and the area under the ROC curve. To compare whether the classifiers have statistically significant performance differences the standard $t$ test is used, comparing the classifiers using accuracy. The analysis has been separated into three main sections: Section 5.7.1 describes how using different measure types affect the performance of the classifiers. The effect of different data mining techniques (classification algorithms) is explained in Section 5.7.2. We see how filters and wrappers may degrade or improve the performance of the classifiers in Section 5.7.3. Before going into these sections we will present the figures that are going to be used in the following sections. Each of the points in the figures represents the average of 10 times stratified 10 fold cross validations performed on the classifiers that have been configured using the same parameters that were outlined in Section 5.6 (Tables Table 5.5 and Table 5.6).

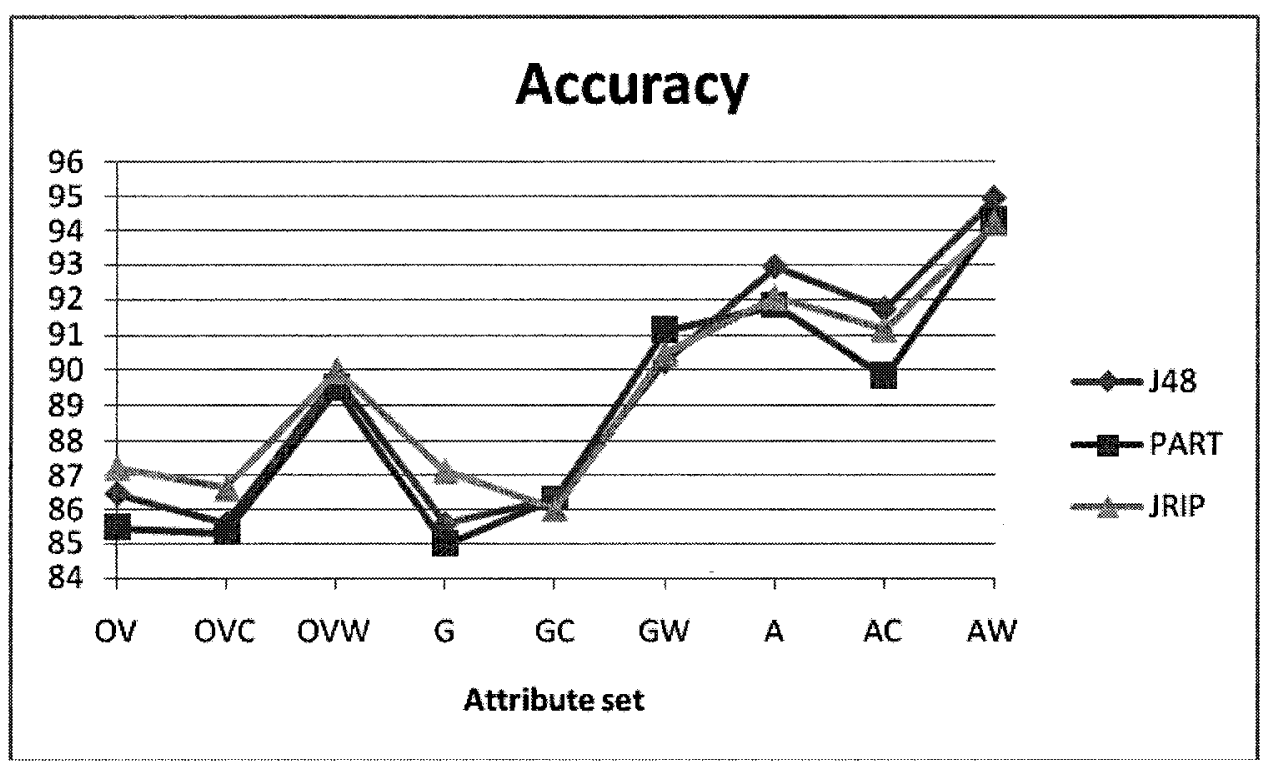

Figure 5.10 - Accuracy of J48, PART and JRIP classifiers versus each attribute set 


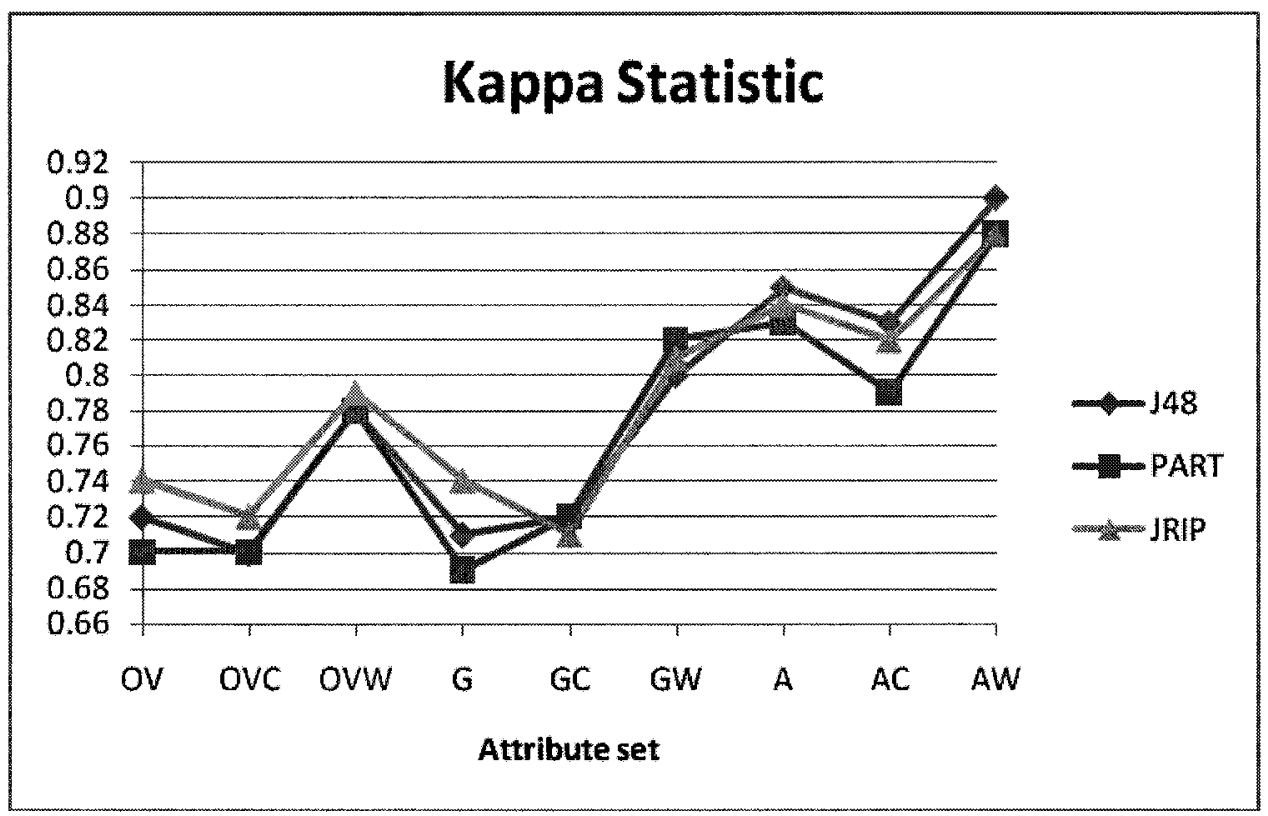

Figure 5.11 - Kappa Statistic of J48, PART and JRIP classifiers versus each attribute set

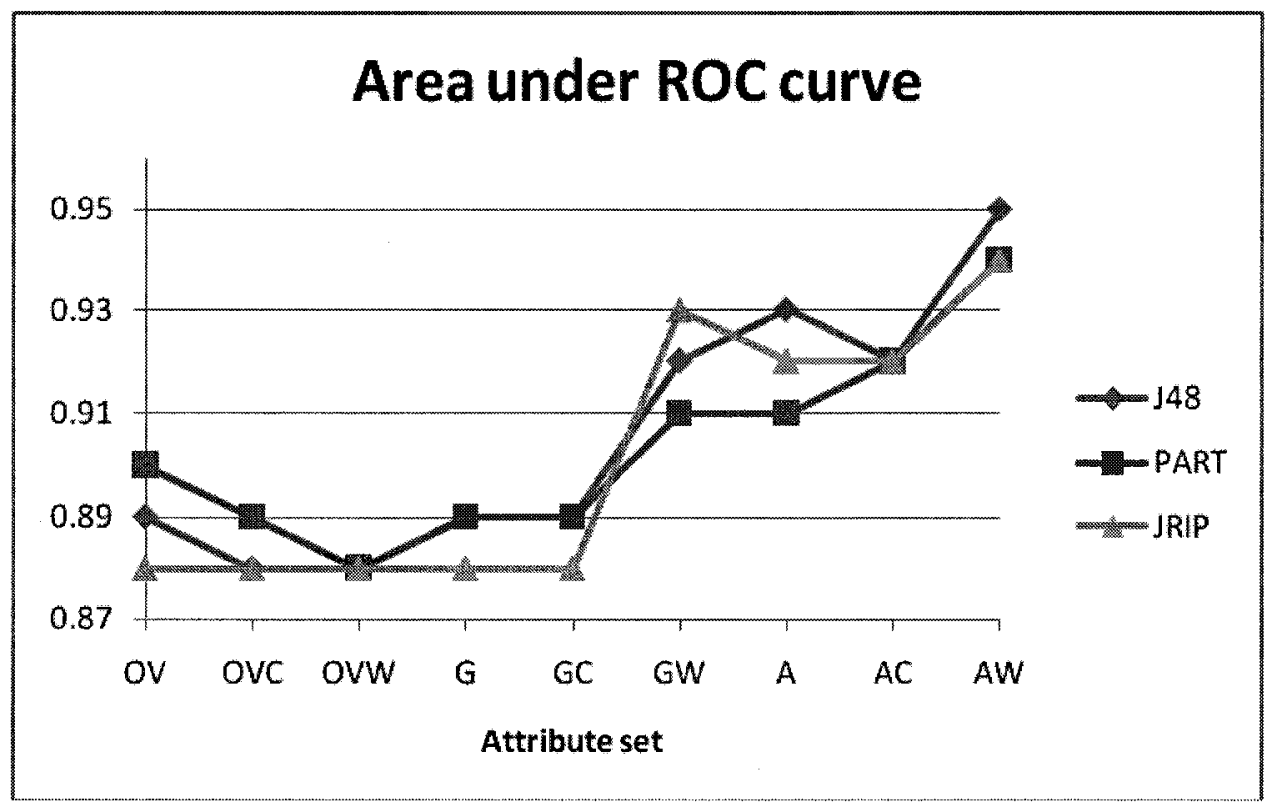

Figure 5.12 - Area under ROC curve of J48, PART and JRIP classifiers versus each attribute set 
As shown by the figures the accuracy and kappa statistic curves follow the same trend for all three classifiers while the area under ROC curve behaves somewhat differently.

\subsubsection{Effect of using different measure types}

In this section, we compare the performance of the classifiers trained with the geometrical measures compared to the simple measures (overlap and volume difference) measures and then investigate how using all the measures will affect the classifier performance using the accuracy performance metric.

As Figure 5.10 shows, the geometrical measures do not show any noticeable performance improvement to just using the simple measures. This is also confirmed with the t-test where no significant performance difference is found between the classifiers trained by geometrical measures as opposed to simple measures. This indicates that using solely geometrical measures may not be required for this case study considering that they result in no performance improvement and also are more complex to implement and test. Also as we will show in Section 5.9 unless a very efficient implementation exists, geometrical measures take significantly more time to execute than the simple measures (in the order of hundreds time slower).

Combining the geometrical and simple measures proves to be very promising though. We see (Table 5.7) significant performance improvements for all three classifiers compared to just using either of the simple (from $4.82 \%$ to $6.47 \%$ improvement) or geometrical measures (from $4.91 \%$ to $7.35 \%$ improvement). This shows that the classifiers achieve

very high discriminating power when taking advantage of both the simple and geometrical measures. 


\begin{tabular}{|l|l|l|l|l|l|}
\hline ClassifierLAttribute Set & Simple & Geometrical & All & $\Delta$ (All-Simple) & $\Delta$ (All-Geometrical) \\
\hline J48 & 86.46 & 85.58 & 92.93 & 6.47 & 7.35 \\
\hline PART & 85.46 & 84.98 & 91.83 & 6.37 & 6.85 \\
\hline JRIP & 87.23 & 87.14 & 92.05 & 4.82 & 4.91 \\
\hline
\end{tabular}

Table 5.7 - Comparison of classifier accuracies with respect to the attribute set category used for training (simple, geometrical, all)

\subsubsection{Effect of using different data mining techniques}

In this section we compare to see if there is any significant performance improvement initiating from the choice of data mining technique. Table 5.8 shows that there is not a noticeable difference in the performance of the classifiers trained with the three data mining techniques. JRIP trains a better classifier when only having geometrical measures available performing $2.16 \%$ better than PART and $1.56 \%$ better than $\mathrm{J} 48$. When using all the measures, regardless of applying the wrapper, $\mathrm{J} 48$ generally performs slightly better. We have not considered the results for the classifiers that are trained with the CFS filtered attribute sets as these classifiers do not achieve any significant improvement over just using the original attribute sets (Section 5.7.3). From Table 5.8, one can conclude that using any of the three data mining techniques would be justifiable except for the fact that one may consider interpreting decision trees easier than rules. Decision trees tend to provide more detail as to how the training instances are categorized by the classifier too. 


\begin{tabular}{|l|l|l|l|l|l|l|}
\hline Attribute Set Classifier & J48 & PART & JRIP & $\Delta($ J48-PART) & $\Delta($ J48-JRIP) & $\Delta$ (PART-JRIP) \\
\hline OV & 86.46 & 85.46 & 87.23 & 1 & Not Sig. & -1.77 \\
\hline OVW & 89.78 & 89.50 & 90.00 & Not Sig. & Not Sig. & Not Sig. \\
\hline G & 85.58 & 84.98 & 87.14 & Not Sig. & -1.56 & -2.16 \\
\hline GW & 90.24 & 91.13 & 90.46 & -0.89 & Not Sig. & Not Sig. \\
\hline A & 92.93 & 91.83 & 92.05 & 1.1 & Not Sig. & Not Sig. \\
\hline AW & 94.92 & 94.32 & 94.21 & 0.6 & 0.71 & Not Sig. \\
\hline
\end{tabular}

Table 5.8 - Comparison of classifier accuracies with respect to the data mining technique (J48, PART, JRIP)

\subsubsection{Effect of using different attribute selection techniques}

The effect of filtering and using wrappers is investigated in this section. Tables Table 5.9, Table 5.10 and Table 5.11 show respectively the classifier accuracies achieved when trained by the simple, geometrical and all measure categories before and after applying the CFS filter and J48 wrapper. We see that using the training instances with only the attributes selected by the wrapper always achieve a significant improvement in the accuracy of the trained classifier compared to using all the attributes; the maximum performance improvement is $7.16 \%$ for the $\mathrm{J} 48$ classifier when using the geometrical measures. On the contrary the CFS filter in some cases significantly degrades the classifier performance. This shows that filters may not improve results at all times. We have used a J48 wrapper here. Using a PART wrapper or a JRIP wrapper also improves classifier performance, but the improvement is not significantly better than the J48 wrapper using any combination of classifierlattribute set, thus we have refrained from reporting the results. 


\begin{tabular}{|l|l|l|l|l|l|}
\hline Classifier\Attribute Set & OV & OVC & OVW & $\Delta($ OVC-OV $)$ & $\Delta($ OVW-OV $)$ \\
\hline J48 & 86.46 & 85.59 & 89.78 & -0.87 & 4.19 \\
\hline PART & 85.46 & 85.30 & 89.50 & Not Sig. & 4.04 \\
\hline JRIP & 87.23 & 86.63 & 90.00 & Not Sig. & 2.77 \\
\hline
\end{tabular}

Table 5.9 - Comparison of classifier accuracies with respect to the use of filters and wrappers on the simple measures for training

\begin{tabular}{|l|l|l|l|l|l|}
\hline ClassifierLAttribute Set & G & GC & GW & $\Delta(\mathbf{G C - G )}$ & $\Delta(\mathbf{G W}-\mathbf{G})$ \\
\hline J48 & 85.58 & 86.31 & 93.24 & Not Sig. & 7.66 \\
\hline PART & 84.98 & 86.31 & 90.13 & 1.33 & 5.15 \\
\hline JRIP & 87.14 & 86.03 & 90.46 & Not Sig. & 3.32 \\
\hline
\end{tabular}

Table 5.10 - Comparison of classifier accuracies with respect to the use of filters and wrappers on the geometrical measures for training

\begin{tabular}{|l|l|l|l|l|l|}
\hline Classifier(Attribute Set & A & AC & AW & $\Delta(\mathbf{A C}-\mathbf{A})$ & $\Delta(\mathbf{A W}-\mathbf{A})$ \\
\hline J48 & 92.93 & 91.71 & 94.92 & -1.22 & 1.99 \\
\hline PART & 91.83 & 89.84 & 94.32 & -1.99 & 2.49 \\
\hline JRIP & 92.05 & 91.17 & 94.21 & Not Sig. & 2.16 \\
\hline
\end{tabular}

Table 5.11 - Comparison of classifier accuracies with respect to the use of filters and wrappers on all of the measures for training 


\subsubsection{Conclusion}

Sections 5.7.1, 5.7.2 and 5.7.3 show that overall the best classifier is obtained by the J48 data mining technique when trained with the complete attribute set (all the measures) removing insignificant attributes at the discretion of the J48 wrapper. Using all the measures improves the performance by a considerably wide margin (around 7\%) compared to just using simple or geometrical measures. Applying the wrapper further improves this by $\sim 2 \%$. Both numbers are proven to be significant by the t-test. Using the J48 classifier and the AW attribute set, we achieve an average accuracy of approximately $95 \%(94.92 \%)$ which is a very reasonable accuracy. This combination results in a kappa statistic of 0.9 and a value of 0.95 for the area under the ROC curve which is close to 1 (i.e. perfect).

\subsection{Tools}

Three main tools have been used in this case study:

1) Left Heart Ventricle Segmentation tool (s): This tool was created by Siemens Corporate Research experts. Each version of this tool implements a version of the image segmentation algorithm. To obtain the segmentations for each version of the image segmentation algorithm the tool had to be run for all of the test cases.

2) MATLAB: We used MATLAB to implement all the comparison measures feeding the segmentations obtained from the left heart ventricle segmentation tool to the comparison measures. In order to calculate the distance maps of the segmentations that are required for the implementation of the geometrical measures, we have used the MATLAB library function bwdist with the Euclidean metric option. This function uses the implementation in [27] to implement the distance transform. To find the boundary of the segmentations, we simply use the gradient function as the gradient at any pixel of a non-boundary pixel is zero (all neighbor pixels are labeled as 1) while it is nonzero in some direction for boundary pixels (a zero-labeled neighbor pixel exists in some direction). MATLAB was also used to obtain the Spearman correlations in Section 5.4. 
3) WEKA: The Waikato Environment for Knowledge Analysis tool was used for obtaining the entire machine learning results. The raw training data was obtained from the output of the MATLAB implemented comparison measures. This is a Java-based tool and is widely used in the research community. It contains the implementation of most of the various data mining techniques and provides an easy to use environment for experimentation.

\subsection{Timing considerations}

As mentioned in the tools section, the comparison measures were implemented in the MATLAB environment. The matrix-oriented environment of MATLAB makes the implementations very concise but MATLAB suffers from being very slow in 3D image processing operations. The graphs in Figure 5.13 show the calculation time of the various comparison measures for each of the 181 segmentation pair comparisons. The time varies based on the size of the segmentation files that have to be compared. Some graphs represent the aggregate time consumption of a few measures. Graph 1 shows the total time of calculating TPVF, FPVF, TC and DSC. Although graphs 1, 4, 5 and 6 show the aggregate calculation time of the measures but as these measures use mostly common operations in their implementations, the time involved in calculating any one of them is comparable with the aggregate time. Table 5.12 shows the average calculation times of the measures. As shown, the Average Distance to Boundary Difference is the fastest measure, followed by the overlap measures (note that we are showing the total time of all the four overlap measures) and the principal axis. The volume difference measures are also very fast to compute. They have not been involved here as those numbers were readily available in this case study and did not require any implementation. The most time-intensive measures are the Hausdorff and Baddeley distances followed by the other geometrical measures. We see a significant gap in calculation times of the majority of the geometrical measures (excluding $\mathrm{ADBD}$ and $\mathrm{PAD}$ ) and the overlaplvolume difference measures. This is due to the fact that the overlap measures do not require any $3 \mathrm{D}$ processing while the geometrical measures all require $3 \mathrm{D}$ processing which MATLAB proves to be very slow at. Also the fact that MATLAB uses an interpreter-based language makes it slower than a compiler based language. Its garbage collection mechanisms do 
not prove to be very effective too as in our experiments large portions of RAM (up to 8 GBs) were allocated at some points of time. It is important to note that these time considerations will be less notable if using an efficient $\mathrm{C}$ implementation of the measures, as the original image segmentation algorithm is implemented in the $\mathrm{C}$ language and is significantly faster than the geometrical measure calculations (the segmentation algorithm takes up to a few 10 s of seconds to output the segmentation of the $3 \mathrm{D}$ volume).
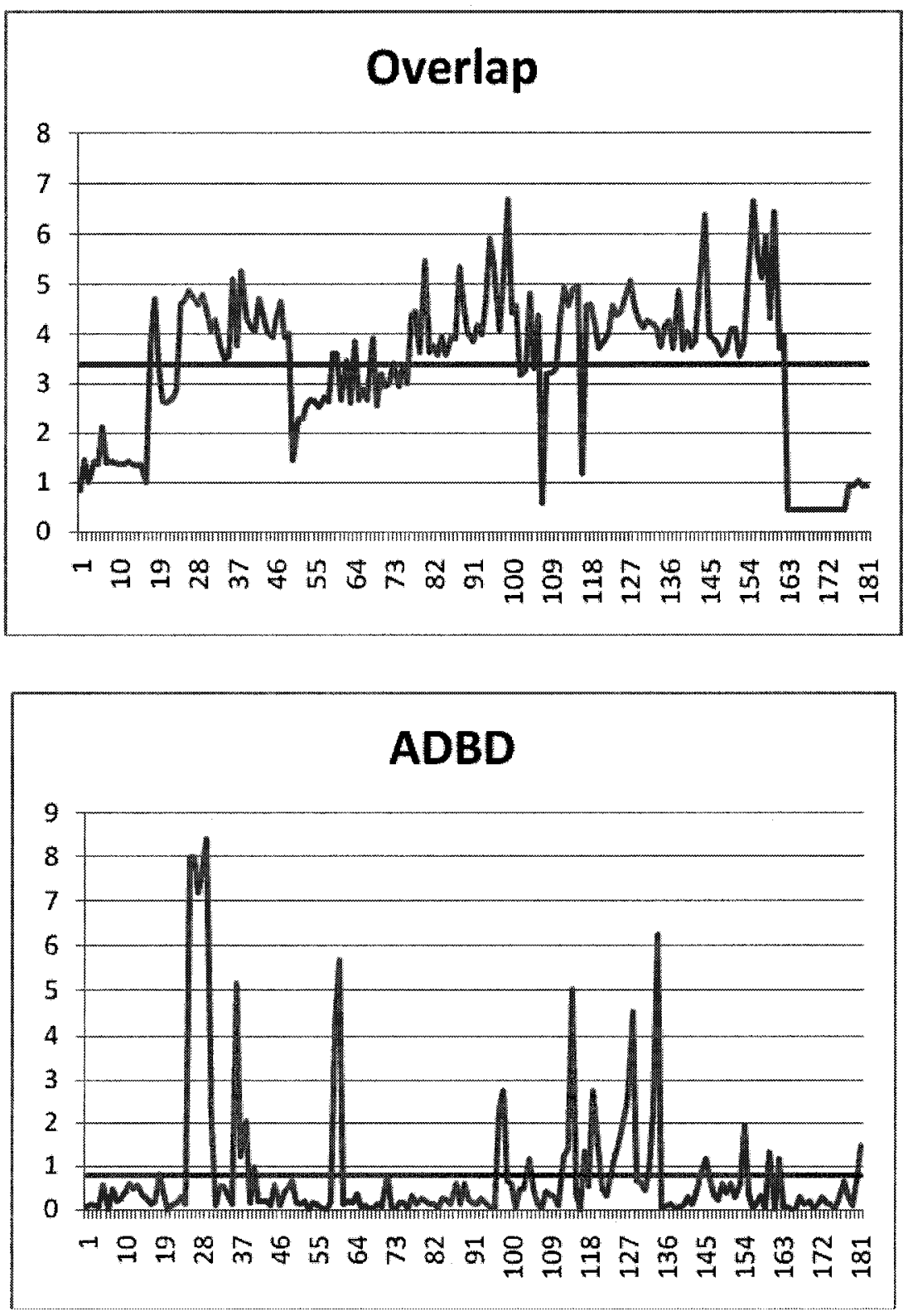

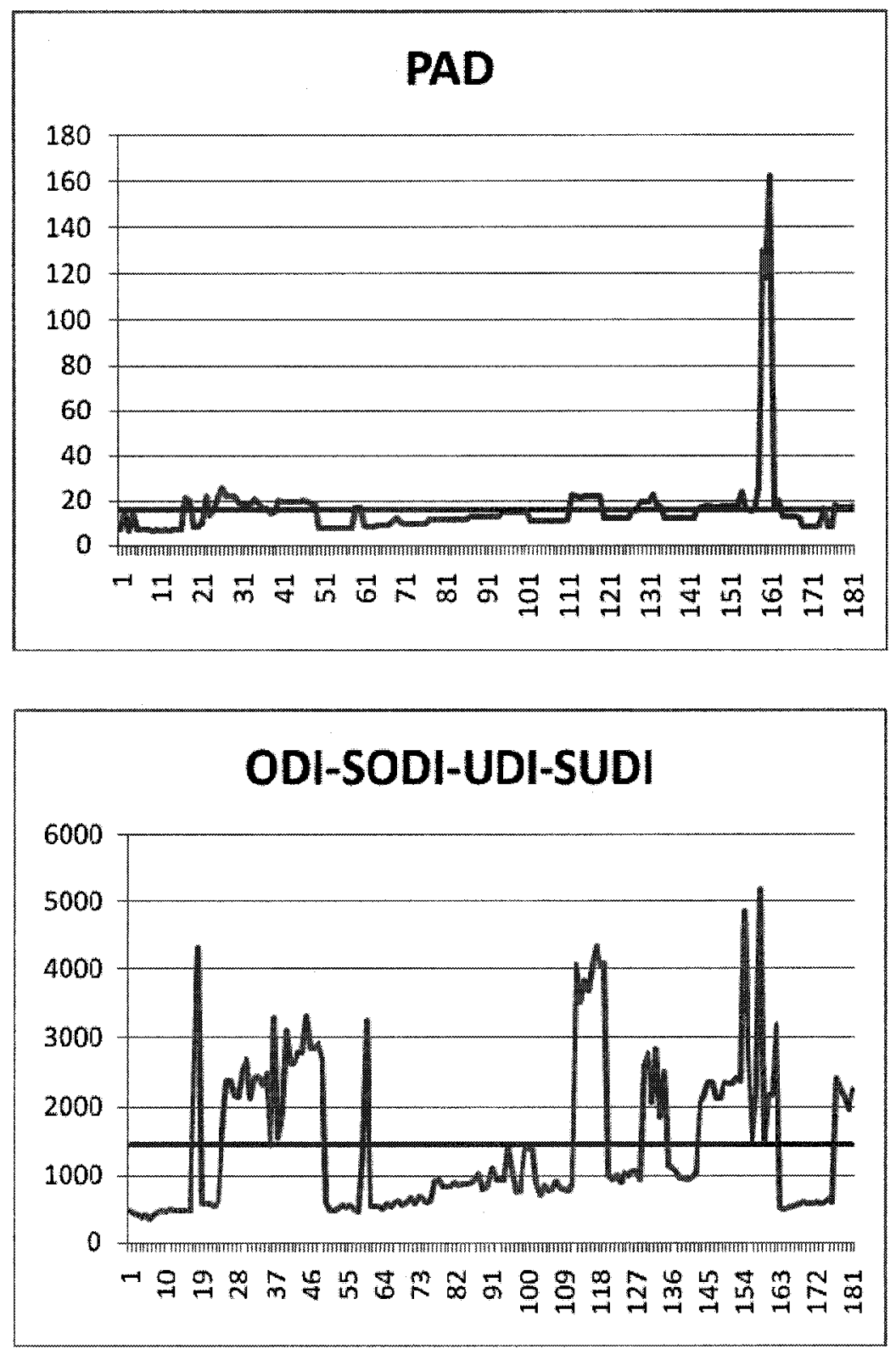

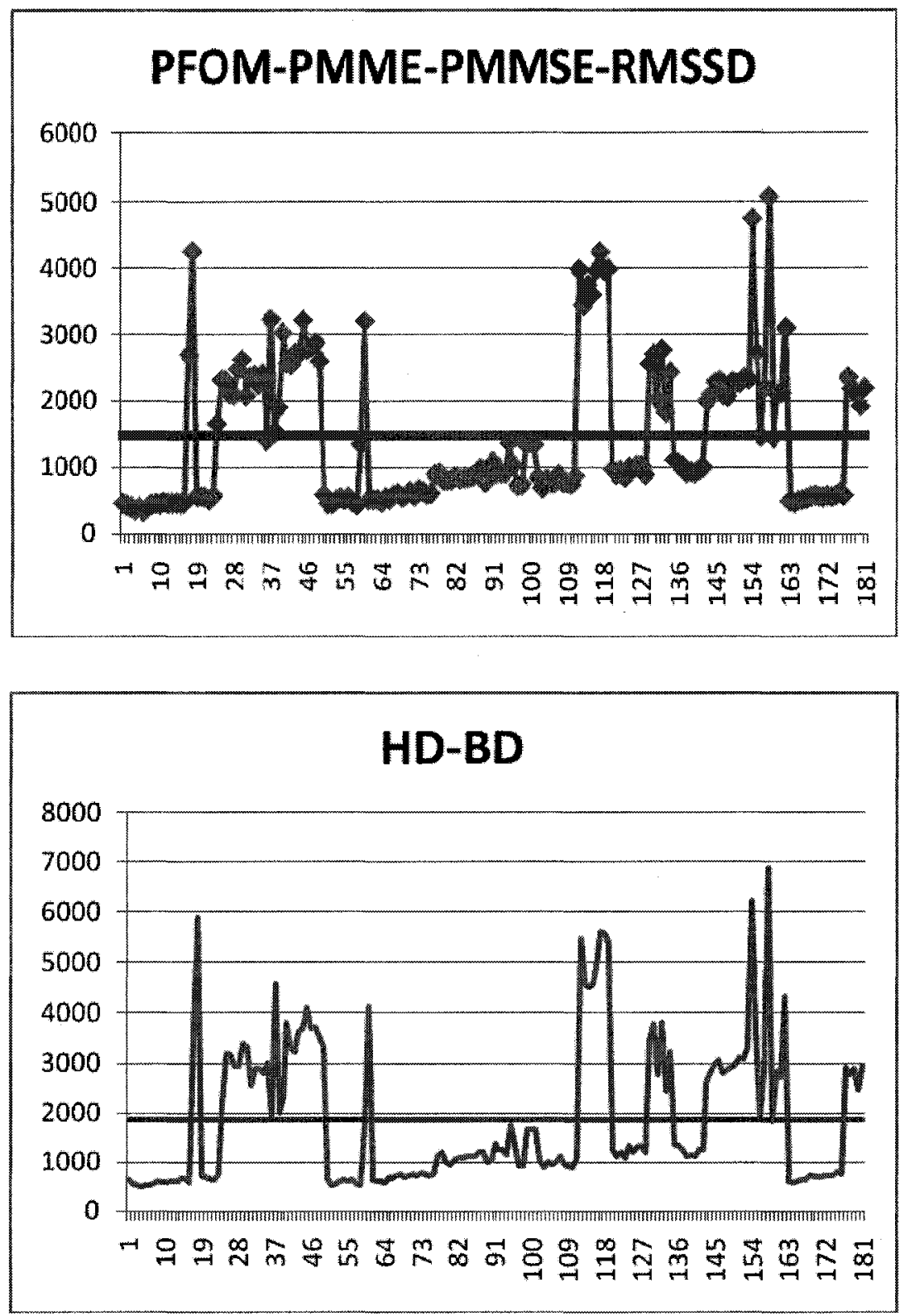

Figure 5.13 - Time consumption of comparison measures $(x-a x i s=$ segmentation pair number, $y$-axis $=$ time consumption in seconds) 


\begin{tabular}{|l|l|}
\hline Comparison Measures & Average Calculation Time (secs) \\
\hline ADBD & 0.80 \\
\hline overlap (TPVF, FPVF, TC, DSC) & 3.39 \\
\hline PAD & 15.62 \\
\hline PFOM, PMME, PMMSE, RMSSD & 1435.57 \\
\hline ODI-SODI-UDI-SUDI & 1465.50 \\
\hline HD-BD & 1853.61 \\
\hline
\end{tabular}

Table 5.12 - Average comparison measure calculation times

Considering the relatively small size of the training set, the classifier construction and classification times were insignificant.

\subsection{Conclusions}

We see that taking advantage of both the simple and geometrical measures results in significantly better performing classifiers compared to using only simple or geometrical measures. The use of wrappers improves the results even further. Measures such as the Tanimoto coefficient (overlap) and SODI (geometrical) prove to be very strong measures for distinguishing consistent pairs of segmentations from inconsistent ones as they are used in the majority of the best performing classifiers. Although all the classifiers perform reasonably well but the J48 classifier is the best performing classifier achieving an approximately $95 \%$ accuracy, 0.9 kappa statistic and 0.95 area under the ROC curve. 


\section{CONCLUSIONS AND FUTURE WORK}

The verification procedure for medical image segmentation algorithms is an iterative process where the same verifications have to be repeated for each version of the image segmentation algorithm manually until the image segmentation algorithm is evaluated to be sufficiently correct for the requirements of the application domain. The segmentation algorithm goes through 10 s of revisions until it is finalized. In this thesis, an approach towards semi-automating this procedure has been proposed. In this approach, the knowledge gained from the manual evaluation of the first few versions (optimally two) of the software under test is captured by calculating similarity measures between the pairs of outputs (segmentations) from different versions of the software under test and finding consistent and inconsistent pairs of outputs. The gathered information is fed into a machine learning algorithm that is used to build a classifier that is able to predict whether an output produced by the current version of the software under test is consistent with the already evaluated outputs of the same input from the previous versions of the software. Simple logic is used to map the consistency classifications from the classifier to the correctness of the output under test (segmentation under test). So effectively an approach has been proposed towards solving the oracle problem. This approach is generic in the sense that the semi-automated procedure described in Chapter 4 can be applied to any software that goes through such an iterative developmentlverification procedure. In this procedure, the machine learning algorithms act as plug and play components and can be replaced with other algorithms if they result in better performance.

The performance of the proposed solution has been investigated on the evaluation of the left heart ventricle segmentation. Though there is room for improvement, the results are very promising and fairly simple classifiers have very good classification accuracies. Using machine learning is very advantageous as it helps to understand which measures are very useful in determining the similarity between two segmentations (or any other output dependant on the application domain) and what ranges of those measures lead to consistent pairs. In this case study it was found that more complex measures such as geometrical measures do not train better classifiers than simple (overlap and volume 
difference) measures, showing that even though these measures contain information about the shape differences between the segmentations, but the simple measures alone perform just as well while consuming considerably less time to implement, test and run. Combining the simple and geometrical measures though, results in statistically significant classifier performance improvements relative to using any of them alone as measured by classifier accuracy. We also see that wrappers further improve the classifier accuracy. Using C4.5 and an attribute set of all the measures that have been filtered by a wrapper, we achieve an accuracy of approximately $95 \%$, a kappa statistic of 0.90 and an area of 0.95 under the ROC curve when only using the training data obtained from the first two revisions of the segmentation algorithm. It is important to note that we have only used machine learning to learn consistent and inconsistent pairs of segmentations from the similarity measures in the oracle design. Trying to learn the qualitative assessment of the medical experts merely from the properties of the medical image segmentation under test may be a very rigorous task and effectively equivalent to learning how to segment the image.

Overall the semi-automated evaluation process proposed in this thesis helps to drastically reduce the time spent in the manual evaluations of the software under test. The issue of the availability of human experts is resolved to a very good extent and as there is less time required for evaluating the software, the software designer has more time to increase the quality of the software which automatically means less revisions of the software under test and less time to get the final revision of the software.

In a nutshell the main contributions of this thesis is devising a re-usable semi-automated approach that attempts to solve the oracle problem for testing (medical) image segmentation algorithms, specifically helping to find the appropriate machine learning techniques and similarity measures pertinent to the application. The proposed approach has only been tried for the evaluation of the left heart ventricle segmentations. Future work will be directed towards testing the performance of the approach in various other applications. Most of the measures defined in this report can be re-used in any type of segmentation evaluation type of application as the measures are not dependant on the left heart ventricle segmentation. Even though the results in this study are promising, but 
more experiments need to be conducted in order to investigate after how many iterations the machine learning algorithm will be able to build a good classifier in other applications. Also trying out this solution during the life cycle of a real-world software project will lead to a better quantitative understanding on the extent of improvement in the quality of the software and the time required for completing the project. Userreadability was the main intention of choosing the classifiers in this research work. An area of research would be to investigate whether more sophisticated machine learning algorithms such as support vector machines and neural networks that produce classifiers that cannot be easily interpretable by humans lead to better accuracies. An area of research that would be of interest to computer vision and medical experts would be to define more similarity measures and using the proposed approach investigate which combination of measures are better indicators of the consistency between a pair of (medical) segmentations. 


\section{REFERENCES}

[1] M. D. Davis and E. J. Weyuker, "Pseudo-oracles for non-testable programs," $A C M, 1981$.

[2] E. J. Weyuker, "On Testing Non-testable Programs," The Computer Journal, vol. 25, no. 4, 1982.

[3] P. Ammann and Jeff Offutt, Introduction to Software Testing, Cambridge University Press, 2008.

[4] A. P. Mathur, Foundations of Software Testing, Addison-Wesley Professional, 2007.

[5] P. D. L. Machado and W. L. Andrade, "The Oracle Problem for Testing against Quantified Properties," QSIC, 2007.

[6] P. E. Ammann and J. C. Knight, "Data Diversity: An Approach to Software Fault Tolerance," IEEE Transactions on Computers, vol. 37, no. 4, 1988.

[7] D. J. Richardson, S. L. Aha and T. O. O'Malley, "Specification-based Test Oracles for Reactive Systems," ACM, 1992.

[8] J. C. Knight and Nancy G. Leveson, "An experimental evaluation of the assumption of independence in multi-version programming," IEEE Transactions on Software Engineering, SE-12(1), pp. 96-109, 1986.

[9] L. C. Briand, Y. Labiche and H. Sun, "Investigating the Use of Analysis Contracts to Improve the Testability of Object-Oriented Code," Software Practice and Experience (Wiley), vol. 33 (7), pp. 637-672, 2003.

[10] T. Gilb, "Software Metrics," New Jersey, 1977.

[11] D. L. Pham, C. Xu, and J. L. Prince, "A Survey of Current Methods in Medical Image Segmentation," Department of Elec. and Comp. Eng., The John Hopkins University, and Laboratory of Personality and Cognition, National Institute on Aging, Technical Report JHU/ECE 99-01, Jan. 1998. 
[12] J. K. Udupa, V. R. LeBlanc, Y. Zhuge, C. Imielinska, H. Schmidt, L. M. Currie, B. E. Hirsch and J. Woodburn, "A framework for evaluating image segmentation algorithms," Elsevier Computerized Medical Imaging and Graphics, vol. 30, pp. 75-87, March 2006.

[13] S. K. Warfield, K. H. Zou, and W. M. Wells, "Simultaneous Truth and Performance Level Estimation (STAPLE): An Algorithm for the Validation of Image Segmentation," IEEE Transactions on Medical Imaging, vol. 23, no. 7, pp. 903-921, July 2004.

[14] W. R. Crum, O. Camara, and D. L. G. Hill, "Generalized overlap measures for evaluation and validation in medical image analysis," IEEE Transactions on Medical Imaging, vol. 25, no. 11, pp. 1451-1461, November 2006.

[15] R. Klette, and A. Rosenfeld, Digital Geometry methods for digital picture analysis, Elsevier, 2004.

[16] C. Rosenberger, S. Chabrier, H. Laurent and B. Emile, "Unsupervised and Supervised Image Segmentation Evaluation," in Advances in Image and Video Segmentation, Y. Zhang, Ed. IGI, 2006, pp. 365-393.

[17] X. Deng et. al, "On Simulating Subjective Evaluation Using Combined Objective Metrics for Validation of 3D Tumor Segmentation," MICCAI, 2007.

[18] C. Zhang and T. Chen, "Efficient Feature Extraction for 2D/3D Objects in Mesh Representations," ICIP, 2001.

[19] H. Zhang, S. Cholleti and S. A. Goldman, "Meta-Evaluation of Image Segmentation Using Machine Learning," IEEE CVPR, 2006.

[20] A. J. Baddeley, "An error metric for binary images," Proceedings of Robust Computer Vision, 1992.

[21] C. Odet, B.Belaroussi and H. Benoit-cattin, "Scalable Discrepancy measures for segmentation evaluation," IEEE ICIP, 2002. 
[22] E. Abdou and W. K. Pratt, "Quantitative Design and Evaluation of Enhancement/Thresholding Edge Detectors," Proceedings of the IEEE, vol. 67, no. 5,1979 .

[23] T. Peli and D. Malah, "A Study of Edge Detection Algorithms," Computer graphics and image processing, 20:1-21, 1982.

[24] I. H. Witten, and E. Frank, Data Mining; Practical Machine Learning Tools and Techniques, Second Edition, Elsevier, 2005.

[25] W. W. Cohen, "Fast Effective Rule Induction," Twelfth International Conference on Machine Learning, pp. 115-123, 1995.

[26] M. A. Hall and G. Holmes, "Benchmarking Attribute Selection Techniques for Discrete Class Data Mining," IEEE Transactions on Knowledge and Data Engineering, vol. 15, no. 3, 2003.

[27] J. H. Friedman, J. L. Bentley and R. A. Finkel, "An Algorithm for Finding Best Matches in Logarithmic Expected Time," ACM Transactions on Mathematical Software, vol. 3, no. 3, pp. 209-226, 1997.

[28] R. V. Hogg, J. McKean and A. T. Craig, Introduction to Mathematical Statistics, Sixth Edition, Prentice Hall, 2004. 